\title{
Microwave Assisted Synthesis of 6-Substituted Aminopurine Analogs in Water
}

\author{
Guirong Qu, ${ }^{*}$ Suhui Han, Zhiguang Zhang, Mingwei Geng and Feng Xue \\ College of Chemistry and Environmental Science, Henan Normal University, Xinxiang 453007, \\ Henan, P. R. China
}

\begin{abstract}
Aminação de derivados de 6-cloropurina em água, assistida por microondas, resultou na preparação de análogos de aminopurina 6-substituídas, em bons rendimentos. Usando um forno de microondas simples, modificado com aparelhagem para refluxo, a aminação do 6-cloro na estrutura da purina ocorreu em condições brandas. Foram preparados 19 análogos conhecidos e 16 desconhecidos de aminopurinas substituídas, através de substituição aromática nucleofílica com filtração simples ou coluna de cromatografia.
\end{abstract}

Microwave assisted amination of 6-chloropurine derivatives with various amines in water resulted in a "green chemistry" protocol for the preparation of 6-substituted aminopurine analogs in very good yields. Using a simply modified microwave oven with the refluxing apparatus, the amination of the 6-chloro in the purine structure occurred smoothly. 19 known and 16 unknown 6 -substituted aminopurine analogs were prepared through nucleophilic aromatic substitution with simple filtration or column chromatography.

Keywords: amination, 6-substituted aminopurine analogs, nucleophilic substitution, microwave irradiation, amines

\section{Introduction}

In modern antiviral and antitumor therapy, an important role is played by modified nucleosides and their analogs, in which modified purine structures are frequently found. ${ }^{1,2} 6$-Substituted aminopurine analogs, the aminated products of the 6-functional groups in purine structures, continued focusing attention due to their wide range of biological activities (inhibitors of Clostridium feseri growth, ${ }^{3}$ cytokinin activity, ${ }^{4}$ CIV-CDK (CIV1) and Candida albicans as antifungal medicines, ${ }^{5}$ selective kinase, ${ }^{6}$ agonists of the $\mathrm{A}_{1}$ adenosine receptor, ${ }^{7}$ the cysteine protease cathepsin $K{ }^{8}$ and platelet aggregation ${ }^{9}$ ). Prominent examples of synthetic 6-substituted aminopurine analogs are $N$-cyclopentyl adenosine (CPA) and $\mathrm{N}$-cyclohexyl adenosine (CHA) (two agonists for the adenosine $\mathrm{A}_{1}$ receptors). ${ }^{10}$

The traditional method for the synthesis of 6substituted aminopurine analogs is the amination of halo, ${ }^{11}$ oxo, ${ }^{12}$ mercapto or methylmercapto ${ }^{13}$ groups with various amines, which can be performed smoothly in organic solvents $\left(\mathrm{BuOH},{ }^{11} \mathrm{CH}_{3} \mathrm{CN},{ }^{13}\right.$ dioxane $,{ }^{14} \mathrm{DMF},{ }^{15}$ or

* e-mail: quguir@yahoo.com.cn
$\left.\mathrm{DMSO}^{16}\right)$ in the presence of tertiary amines $\left(\mathrm{Et}_{3} \mathrm{~N}, \mathrm{~N}, \mathrm{~N}\right.$ dimethyl cyclohexylamine or diisopropylethylamine) and the catalysts $\left(\mathrm{P}_{2} \mathrm{O}_{5},{ }^{14} \mathrm{~K}_{2} \mathrm{CO}_{3}\right.$ and $\left.\mathrm{Cu} / \mathrm{K}_{3} \mathrm{PO}_{4}{ }^{16}\right)$. However, the complete conversion usually needs $2-24 \mathrm{~h}$ and the use of toxic organic solvents or relatively expensive reagents could not be avoided, which dose not conform to the requirement of modern pharmaceutical industry and environmental friendliness.

In any case, the development of efficient protocols for the synthesis of aminopurine analogs is still an important goal. Mild reaction conditions, short reaction times and high selectivity and yields are preferable, which makes us pay attention to microwave irradiation. Microwave assisted organic synthesis and functional group conversions have been an increasingly popular field as indicated by numerous publications in the past few years. ${ }^{17}$ It often leads to rate enhancement, higher yields, easier work-up and better selectivity as well as shortening reaction times compared with the conventional heating methods. In spite of the body of literature about microwave accelerated organic synthesis, the references on the microwave-assisted synthesis of nucleoside compounds are only a few examples. In our previous research, we have prepared a series of modified nucleosides under microwave irradiation. ${ }^{18}$ 
To avoid the contamination of organic solvents, we turn to water. Water as a kind of environmentally benign solvent for organic reactions has attracted more and more attention in recent years. ${ }^{19}$ Synthesis using water as the solvent has several advantages, such as low toxicity, low cost, high yields and ease of manipulation..$^{20,21}$

In this paper, we described a facile, efficient and ecofriendly protocol for the preparation of 6-substituted aminopurine analogs based on the nucleophilic substitution of 6-chloropurine derivatives with various amines in water under microwave irradiation. Using this green method, we obtained 19 known and 16 unknown 6-substituted aminopurine analog compounds in moderate to high yields.

\section{Results and Discussion}

In view of the limitations of the existing methods, a study of microwave assisted synthesis of 6-substituted aminopurine analogs in water using a simply modified microwave oven with the refluxing apparatus was undertaken. First of all the amination of model 6chloropurine $\mathbf{1}$ with aniline producing $\mathbf{1 b}$ by conventional heating methods was reexamined (Scheme 1).

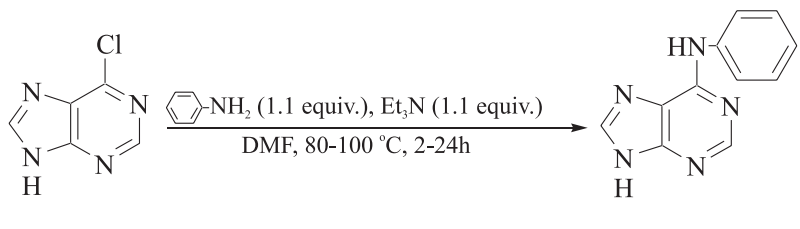

1

$1 \mathbf{b}$

Scheme 1. Amination of $\mathbf{1}$ with aniline in the original condition.

As shown in Scheme 1, the original conditions involved the use of $\mathrm{Et}_{3} \mathrm{~N}$ (1.1 equiv.) and the keeping of the reaction mixture in DMF at $80-100{ }^{\circ} \mathrm{C}$ for $2-24 \mathrm{~h}$. Based on these conditions, different solvents and different equivalents of aniline and $\mathrm{Et}_{3} \mathrm{~N}$ to the precursor 6-chloropurine $\mathbf{1}$ were tested, widening the set of parameters (Table 1).

Table 1 summarized the studies carried out into variation of these conditions. In the original condition, the maximum conversion was reached at approximately $18 \mathrm{~h}$ and $80{ }^{\circ} \mathrm{C}$ (compare entries 1, 2, 3 and 4 in Table 1), so we fixed $80{ }^{\circ} \mathrm{C}$ and $18 \mathrm{~h}$ as the reaction temperature and reaction time to test the other conditions. It was quickly proved that the presence of $\mathrm{Et}_{3} \mathrm{~N}$ was unnecessary. We found $\mathbf{1 b}$ could be also obtained in good yields when the equivalent of aniline was increased to 5 (compare entries 6, 9, 11, 12 in Table 1) but in lower yields when it was remained to 1.1 (entries 5 and 8) in the absence of $\mathrm{Et}_{3} \mathrm{~N}$. The results showed that the presence of $\mathrm{Et}_{3} \mathrm{~N}$ strengthened the base of the reaction mixture and the nucleophilicity of aniline, which could be complemented by the enhanced amount of aniline.

The solvent was examined subsequently. As shown in Table 1 (entries 6, 9, 11, 12), the solvent could be replaced by $\mathrm{H}_{2} \mathrm{O}$ and the product $\mathbf{1 b}$ was still formed in good yield. The possible explanation is that the polarity of water is lower than DMF and higher than $\mathrm{BuOH}$ and EtOH, which makes water an appropriate medium to allow $\mathbf{1 b}$ to crystallize from the reaction system. The experimental fact proved it that 6-chloropurine 1 solved completely after the addition of colorless aniline to its suspension in water and $\mathbf{1 b}$ precipitated out from the aqueous solution because of lower polarity and solubility of $\mathbf{1 b}$ in water than that of $\mathbf{1}$.

Finally, the amount of aniline was examined. Although $\mathbf{1 b}$ formed in good yields as the equivalent of aniline to $\mathbf{1}$ was 5, there was unreacted aniline left and need to wash many times, which made some loss of $\mathbf{1 b}$. The excellent results were obtained when the equivalent was 3 (compare entries 9 and 10 in Table 1).

Table 1. Influence of variation of conditions on the yields $\mathbf{1 b}$

\begin{tabular}{|c|c|c|c|c|c|c|}
\hline Entry & Aniline equiv. & $\mathrm{Et}_{3} \mathrm{~N}$ equiv. & Solvent & Reaction $\mathrm{T} /{ }^{\circ} \mathrm{C}$ & time/h & Yield/ $\%$ a \\
\hline 1 & 1.1 & 1.1 & DMF & 80 & 12 & 65 \\
\hline 2 & 1.1 & 1.1 & DMF & 80 & 18 & 71 \\
\hline 3 & 1.1 & 1.1 & $\mathrm{DMF}$ & 80 & 24 & 68 \\
\hline 4 & 1.1 & 1.1 & DMF & 100 & 18 & 63 \\
\hline 5 & 1.1 & 0 & DMF & 80 & 18 & 52 \\
\hline 6 & 5 & 0 & DMF & 80 & 18 & 73 \\
\hline 7 & 1.1 & 1.1 & $\mathrm{H}_{2} \mathrm{O}$ & 80 & 18 & 79 \\
\hline 8 & 1.1 & 0 & $\mathrm{H}_{2}^{2} \mathrm{O}$ & 80 & 18 & 56 \\
\hline 9 & 5 & 0 & $\mathrm{H}_{2}^{2} \mathrm{O}$ & 80 & 18 & 80 \\
\hline 10 & 3 & 0 & $\mathrm{H}_{2}^{2} \mathrm{O}$ & 80 & 18 & 83 \\
\hline 11 & 5 & 0 & $\mathrm{BuOH}^{2}$ & 80 & 18 & 70 \\
\hline 12 & 5 & 0 & $\mathrm{EtOH}$ & 80 & 18 & 71 \\
\hline $13^{\mathrm{b}}$ & 3 & 0 & $\mathrm{H}_{2} \mathrm{O}$ & 72 & $10 \mathrm{~min}$ & 87 \\
\hline $14^{\mathrm{b}}$ & 3 & 0 & $\mathrm{H}_{2}^{2} \mathrm{O}$ & 72 & $8 \mathrm{~min}$ & 85 \\
\hline
\end{tabular}

${ }^{\mathrm{a}}$ Isolated yield. ${ }^{\mathrm{b}}$ Microwave Irradiation $\left(200 \mathrm{~W}, 72^{\circ} \mathrm{C}\right)$. The temperature was measured immediately after the irradiation. 
Running the reaction with 3 equiv. aniline in water under microwave irradiation appeared to proceed more efficiently (entries 13 and 14 in Table 1). At 200W for 2 min, the reaction mixture began to boil and could be condensed by the refluxing apparatus. The attempt to increase microwave power in order to reduce reaction time is unsuccessful because the reaction vapor could not be condensed as soon as possible with the elevated temperature quickly, which would increase the possibility of the release of aniline to the air.

After some experiments, the best procedure for the irradiation is described as follows. The reaction mixtures were irradiated successively for 2.5 min periods followed by a 3 min cooling interval between irradiations. This method was designed to avoid overheating of reactants since the simply modified microwave oven lacks the attributes of stirring and temperature control.

After fixed the procedure, irradiation time was tested (Table 2). Inspection of data showed the conversion increased with the prolonging of reaction time and the best result was achieved between 8 and $10 \mathrm{~min}$. Compared Table 1 and 2, the conclusion can be easily reached that microwave assisted synthesis of 6-phenylaminopurine $\mathbf{1 b}$ can reduce the long reaction time of conventional thermal methods to $10 \mathrm{~min}$.

Table 2. Influence of different irradiation time on the yields $\mathbf{1 b}$ in water

\begin{tabular}{lccc}
\hline Entry & Aniline equiv. & Irradiation time/min & Isolated yield/\% \\
\hline 1 & 3 & 5 & 72 \\
2 & 3 & 8 & 85 \\
3 & 3 & 10 & 87 \\
4 & 3 & 13 & 84 \\
\hline
\end{tabular}

Having established a simplified reaction protocol (Scheme 2), we applied the modified conditions to a series of 6-chloropurine derivatives (1-9) and various amines (10a-e) (Table 3). For comparison most reactions were run for the same irradiation power (200W) and time (10min). The by-product of these reactions, hydrogen

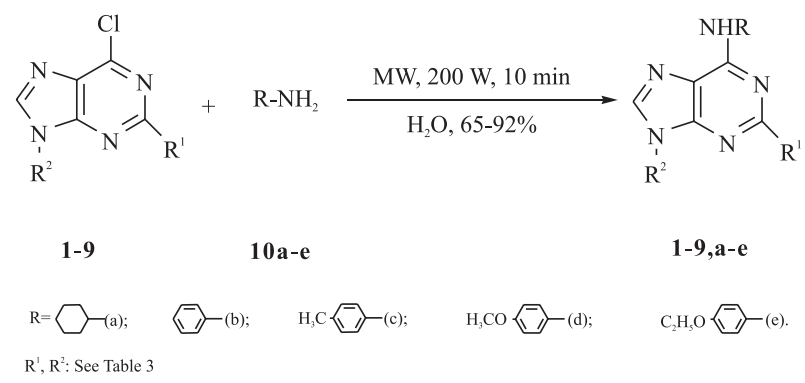

Scheme 2. The synthesis of 6-substituted aminopurine analogs 1-9,a-e by the modified amination protocol of 6-chloropurine derivatives 1-9 with various amines 10a-e. chloride, was quenched by amines, used as nucleophiles and bases, forming ammonium salts, thus avoiding its release into the environment. The existence of ammonium salts was not an obstacle to the purification of 6-substituted aminopurine analogs because the latter are the crystalline solids, which are poorly soluble in water and can be separated easily from the former by direct filtration.

We found this protocol allowed 6-substituted aminopurine 1-3,a-e to be isolated with moderate to high yields (entries

Table 3. Amination of 6-chloropurine derivatives 1-9 with various primary amines 10a-e in water under microwave irradiation

\begin{tabular}{|c|c|c|c|}
\hline Entry & $\begin{array}{l}\text { 6-chloropurine } \\
\text { derivatives }\end{array}$ & $\begin{array}{c}\text { 6-substituted } \\
\text { aminopurine analogs }\end{array}$ & Yield $/ \%^{a}$ \\
\hline 1 & & 1a & 79 \\
\hline 2 & & $1 b$ & 87 \\
\hline 3 & & $1 c$ & 85 \\
\hline 4 & $\begin{array}{ll}\mathrm{H} \\
\mathrm{N}\end{array}$ & 1d & 82 \\
\hline 5 & & $1 e$ & 81 \\
\hline 6 & & $2 a$ & 90 \\
\hline 7 & & $2 b$ & 87 \\
\hline 8 & & $2 c$ & 82 \\
\hline 9 & & $2 d$ & 88 \\
\hline 10 & & $3 a$ & 78 \\
\hline 11 & & $3 b$ & 72 \\
\hline 12 & & $3 c$ & 65 \\
\hline 13 & & 3d & 68 \\
\hline 14 & & $3 e$ & 70 \\
\hline 15 & & $4 a$ & 86 \\
\hline 16 & & $4 b$ & 92 \\
\hline 17 & & $4 c$ & 82 \\
\hline 18 & & $4 d$ & 83 \\
\hline 19 & & $5 a$ & 80 \\
\hline 20 & & $5 b$ & 89 \\
\hline 21 & & $5 c$ & 91 \\
\hline 22 & & $5 d$ & 83 \\
\hline 23 & $\begin{array}{c}\mathrm{CH}_{2} \mathrm{CN} \\
\mathbf{5}\end{array}$ & $5 e$ & 85 \\
\hline 24 & & $6 a$ & 82 \\
\hline 25 & & $6 b$ & 80 \\
\hline 26 & & $6 c$ & 81 \\
\hline 27 & $\mathrm{CH}_{2} \mathrm{CH}_{2} \mathrm{CN}$ & 6d & 81 \\
\hline 28 & 6 & $6 e$ & 79 \\
\hline 29 & & $7 a$ & 87 \\
\hline 30 & & $7 b$ & 91 \\
\hline 31 & & $7 c$ & 88 \\
\hline 32 & & 7d & 85 \\
\hline 33 & $\mathrm{OH} \mathrm{OH}$ & $7 e$ & 82 \\
\hline 34 & & $8 \mathbf{a}$ & $34^{\mathrm{b}}$ \\
\hline & & $8 a^{\prime}$ & 26 \\
\hline 35 & & $9 a$ & $42^{\mathrm{b}}$ \\
\hline
\end{tabular}

a Isolated yield. 'Deacetylated products. 
1-14 in Table 2). For 6-chloropurine 1 and 2,6-dichloropurine $\mathbf{2}$, on adding the amines to their suspension a clean solution and a heterogeneous oil phase at the bottom of the flask could be obtained because the amines helped $\mathbf{1}$ and $\mathbf{2}$ solve in water. When irradiated, the reaction mixture boiled within a few seconds and the two phases merged well and reacted quickly. The refluxing apparatus made the reaction vapor and the amines with low boiling points return to the reaction systems. Then the mixtures were cooled to room temperature and some solids crystallized slowly. After filtering and washing, the crystals obtained showed short melting ranges and comprehensive attributes of amine and purine structure in NMR and IR, which indicated the desired products 1a-e and $\mathbf{2 a - d}$ were prepared. After the mixture of $\mathbf{2}$ and p-ethoxyphenyl amine 10e was irradiated, a white solid emerged which polarity was so high that it could not solve even in DMF and DMSO, so it was given up. For 2-amino6-chloropurine $\mathbf{3}$, after the addition of amines and during the irradiation, no clean solutions were obtained except the reaction with cyclohexylamine 10a. 3a crystallized slowly from the solution after cooled to room temperature about 30 min, but $\mathbf{3 b}$-e were formed during the reaction, which could be proved by NMR that the solids filtered completely were expected products.

We prepared 6-chloro substituted purine acyclic nucleosides 4, 5, 6 and 9 according to the reported methods, ${ }^{22}$ and applied 4-6 to our aminating protocol, then obtained the desired acyclic 6-substituted aminopurine nucleosides 4-6,a-e in high yields after column chromatography purification (entries 15-28 in Table 3). The products 4-6,a-e are new compounds and their structures, proven by NMR, IR and MS, are described in the experimental section and Supplementary Information. Under the same conditions, 6chloropurine nucleoside 7 obtained 7a-e in $82-91 \%$ after simply filtration (entries 29-33 in Table 3). As 2', 3', 5'-triacetyl-2,6-dichloropurine nucleoside 8 and 2,6dichloro-9-[(2-acetoxylethoxy) methyl]purine 9 reacted with 10a, the deacetylated products $\mathbf{8 a}$ (entry 34) and 9a (entry 35 in Table 3 ) were prepared and no satisfactory results were given: ( $i$ ) No obvious reaction was monitored by TLC immediately after the irradiation; (ii) On a long time standing (2 days), there is no any solid precipitated out. TLC showed the disappearance of $\mathbf{8}$ and the appearance of deacetylated product $\mathbf{8 a}$ and acetylated product 8a'. And for 9, there was about half conversion to the desired product 9a. After chromatography, 8a, 8a' and 9a were isolated in rather low yields $(26-42 \%)$. NMR proved the replacement of -OAc to $-\mathrm{OH}$ and 6-chloro to 6-cyclohexylamino, which made $8 \mathbf{a}$ and $9 \mathbf{a}$ easily solve in water because of their increased polarity compared to $\mathbf{8}$ and $\mathbf{9}$ and was the possible reason to the above experimental phenomena. It indicated that the differences of solubility in water between the starting materials and the products played an important role when water acted as the reaction medium.

\section{Conclusions}

In conclusion, we reported an environmentally benign microwave-assisted protocol for the rapid and direct amination of 6-chloropurine derivatives to prepare 6-substituted aminopurine analogs $\mathbf{1 - 7}$,a-e in $10 \mathrm{~min}$ via nucleophilic substitution, making full use of the soluble differences in water between the starting materials and the products. Products with moderate to high purity were isolated by filtration directly and column chromatography. The by-product, hydrogen chloride, is quenched as an ammonium salt in the course of the reaction, avoiding its release to the air, which presents an additional environmentally friendly synthetic advantage.

\section{Experimental}

${ }^{1} \mathrm{H}$ and ${ }^{13} \mathrm{C}$ NMR spectra were recorded in DMSO- $d_{6}$ solutions on a Bruker DPX-400 spectrometer (at 400 $\mathrm{MHz}$ and $100 \mathrm{MHz}$, respectively) using TMS as internal standard. Chemical shifts $(\delta)$ are reported in ppm and coupling constants $(J)$ are given in Hz. IR spectra were recorded on a Bruker Vector 22 spectrometer, using $\mathrm{KBr}$ tablets, and the frequency being expressed in $\mathrm{cm}^{-1}$. Mass spectra (ESI) were recorded on an Agilent 1100 (LCMSD-Tarp-SL) mass spectrometer. Elemental analyses were performed on an EA-1110 (CE Instruments) instrument. Melting points were determined with an $\mathrm{XRC}-1$ micro melting point apparatus and are uncorrected. TLC analysis was carried out on silica gel plates (Merck, silica gel $60 \mathrm{~F}_{254}$ ) and column chromatography was performed on silica gel (Merck, 200-300 mesh). All the reactions were carried out in a simply modified microwave oven (SANYO EM-202MSI, $2450 \mathrm{MHz}$ ) attaching the refluxing apparatus. A hole is designed in the microwave oven through which a roundbottomed flask is fixed and can be refluxed directly. Irradiation time can be prefixed by the time apparatus and output power can be shown in the electric current bell according to the working curve of the oven. For example, $90 \mathrm{~mA}$ in electric current bell stands for output power $200 \mathrm{~W}$. The reaction temperatures are measured immediately after the stop of irradiation by putting the thermometer into the reaction mixtures. 
Procedure for the optimization of the amination conditions

6-chloropurine $1(0.3 \mathrm{~g}, 2 \mathrm{mmol})$ is dissolved in $10 \mathrm{~mL}$ of different solvents (see Table 1) in a $50 \mathrm{~mL}$ round-bottomed flask and aniline is added to the suspension, which equivalents are $1.1(0.22 \mathrm{~mL}, 0.22 \mathrm{mmol}), 3(0.6 \mathrm{~mL}, 0.6 \mathrm{mmol}), 5(1.0$ $\mathrm{mL}, 10 \mathrm{mmol})$, respectively. Different amount of $\mathrm{Et}_{3} \mathrm{~N}$ is added as the design in Table 1, and then keep the oil bath temperature to $80^{\circ} \mathrm{C}$ (or $100^{\circ} \mathrm{C}$ ) for $12 \mathrm{~h}, 18 \mathrm{~h}$ or $24 \mathrm{~h}$. After the reaction, the mixtures (DMF, $\mathrm{BuOH}, \mathrm{EtOH}$ as the solvents) are concentrated in vacuum. As a result, they become thicker and their colors become deeper (red for DMF and yellow for $\mathrm{BuOH}, \mathrm{EtOH}$ ). Add appropriate amount silica gel (Merck, 200-300 mesh) to the concentrated reaction mixtures and make them disperse well, and then purify by column chromatography with $\mathrm{CHCl}_{3} / \mathrm{CH}_{3} \mathrm{OH}(9 / 1, \mathrm{v} / \mathrm{v})$. Solid can be filtered from the mixtures and the filtrate is concentrated to $1 / 3$ volume, some products crystallized again and collected. The yields are listed in Table 1.

Procedure for the optimization of irradiation time and power

On adding $0.6 \mathrm{~mL}$ aniline ( 3 equiv.) to the suspension of $0.3 \mathrm{~g} 1$ in $10 \mathrm{~mL}$ water, a clean solution obtained as well as a heterogeneous oil phase at the bottom of the flask. The flask is fixed to the microwave oven and irradiated successively for 2.5 min periods followed by a 3 min cooling interval between irradiation at $200 \mathrm{~W}$ for 5 $\mathrm{min}, 8 \mathrm{~min}, 10 \mathrm{~min}$ and $13 \mathrm{~min}$ (that is the irradiation is stopped at the second, third, fourth and fifth interval). Different amounts of product $\mathbf{1 b}$ are obtained and the yields are listed in Table 2.

When irradiated at the power of above $200 \mathrm{~W}$, the reaction mixture boiled violently within 40 seconds and cannot be condensed to return to the flask as soon as possible, which made it spill to the air. To avoid that, we choose $200 \mathrm{~W}$ as the irradiation power.

General procedure for the synthesis of 6-substituted aminopurine analogs 1-7, a-e and 8a, 8a', 9a

Amine $(6 \mathrm{mmol})$ is added to a stirred suspension of 6-chloropurine derivatives 1-9 $(2 \mathrm{mmol})$ in water $(10 \mathrm{~mL})$ in a $50 \mathrm{~mL}$ round-bottomed flask. After vibration, the flask is moved into microwave oven and irradiated at $200 \mathrm{~W}$ for 10 minutes. At each interval, TLC monitors the reaction progress. When the reaction completed, the mixture is cooled to room temperature and desired 6-substituted aminopurine analogs 1-3,a-e and 7a-e precipitate out. Then filter the solid directly followed by washing with cold water $(3 \times 5 \mathrm{~mL})$. The filtrate is concentrated to $1 / 3$ volume, and collects the products crystallized again from the mixture. The products 4-6,a-e and 8a, 8a', 9a are purified by column chromatography with $\mathrm{CHCl}_{3} / \mathrm{CH}_{3} \mathrm{OH}$ (9/1, v/v) for 4-6,a-e and $\mathrm{CHCl}_{3} / \mathrm{CH}_{3} \mathrm{OH}(97 / 3, \mathrm{v} / \mathrm{v})$ for $\mathbf{8 a}, 8 \mathbf{a}^{\prime}, 9 \mathbf{a}$ after the concentration of the reaction mixtures.

Physical data of the known compounds had been partly reported in the literatures ${ }^{4,23-26}$ and melting points of $\mathbf{2 a}$ and $3 \mathbf{a}$ are not identified with the literatures ${ }^{4,23}$ although the NMR spectra have proved their structures.

\section{Spectroscopic data of new compounds}

Note: Assignments of $\mathrm{H}$ and $\mathrm{C}$ are given according to purine nucleoside numbering:

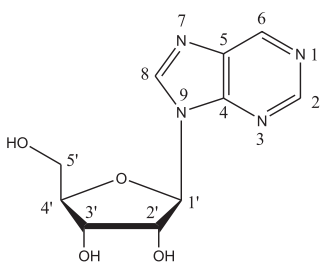

9- $\beta$-cyanoethyl-6-cyclohexylamino purine $(4 a)$. White needle crystal; mp $137-139{ }^{\circ} \mathrm{C} .{ }^{1} \mathrm{H}$ NMR (DMSO- $\left.d_{6}\right) \delta$ 1.112-1.881 (m, 10H, H cyclohexyl), 3.168 (t, $2 \mathrm{H}, J 6.4 \mathrm{~Hz}$, $\mathrm{CH}_{2} \mathrm{CH}_{2} \mathrm{CN}$ ), 4.101 (br, 1H, H cyclohexyl), 4.447 (t, 2H, $J$ 6.4Hz, $\left.\mathrm{NCH}_{2} \mathrm{CH}_{2}\right), 7.554$ (d, $\left.1 \mathrm{H}, J 8.0 \mathrm{~Hz}, \mathrm{NH}\right), 8.187$ (s, 1H, H-2), 8.219 (s, $1 \mathrm{H}, \mathrm{H}-8$ ). ${ }^{13} \mathrm{C}$ NMR (DMSO- $d_{6}$ ) $\delta$ $18.56\left(\mathrm{CH}_{2} \mathrm{CN}\right), 39.26\left(\mathrm{NCH}_{2} \mathrm{CH}_{2}\right), 24.80,25.56,26.25$, 32.78, 33.35, 49.11 (C cyclohexy), $118.72(\mathrm{CN}), 119.33$ (5-C), 140.59 (8-C), 149.18 (6-C), 153.03 (4-C), 154.30 (2-C). MS (ESI) $m / z$ [ $\left[\mathrm{M}^{+} \mathrm{Na}^{+}-1,292.8\right], 270.8,254.8$, 228.8, 203.7, 150.8. IR (KBr) $v_{\max } / \mathrm{cm}^{-1}: 3398,3385,3084$, 3036, 2933, 2854, 2250, 1607, 1586, 1475, 1366, 1299, 772. Anal. Calc. for $\mathrm{C}_{14} \mathrm{H}_{18} \mathrm{~N}_{6}$ : C, 62.22; H, 6.67; N, 31.11 . Found: C, 61.97; H, 6.73; N, $31.34 \%$.

9- $\beta$-cyanoethyl-6-phenylamino purine (4b). Lustrous flakes; mp $168-170{ }^{\circ} \mathrm{C} .{ }^{1} \mathrm{H}$ NMR (DMSO- $d_{6}$ ) $\delta 3.229$ (t, $2 \mathrm{H}, J 6.4 \mathrm{~Hz}, \mathrm{CH}_{2} \mathrm{CH}_{2} \mathrm{CN}$ ), 4.538 (t, $2 \mathrm{H}, J 6.4 \mathrm{~Hz}$, $\left.\mathrm{NCH}_{2} \mathrm{CH}_{2}\right), 7.049\left(\mathrm{t}, 1 \mathrm{H}, J 6.4 \mathrm{~Hz}, \mathrm{H}_{\mathrm{Ar}}\right), 7.340(\mathrm{t}, 2 \mathrm{H}, J$ $\left.6.0 \mathrm{~Hz}, \mathrm{H}_{\mathrm{Ar}}\right), 7.972\left(\mathrm{~d}, 2 \mathrm{H}, J 8.8 \mathrm{~Hz}, \mathrm{H}_{\mathrm{Ar}}\right), 8.390(\mathrm{~s}, 1 \mathrm{H}$, $\mathrm{H}-2), 8.445$ (s, 1H, H-8), 9.917 (s, 1H, NH). ${ }^{13} \mathrm{C}$ NMR $\left(\mathrm{DMSO}-d_{6}\right) \delta 18.63\left(\mathrm{CH}_{2} \mathrm{CN}\right), 39.46\left(\mathrm{NCH}_{2} \mathrm{CH}_{2}\right)$, $118.71(\mathrm{CN}), 120.20(\mathrm{C}-5), 121.33,123.10,128.82$, $140.06\left(\mathrm{C}_{\mathrm{Ar}}\right), 141.94(\mathrm{C}-8), 149.75$ (C-6), 150.04 (C4), 152.53 (C-2). MS (ESI) $\mathrm{m} / z$ [ $\left.\mathrm{M}^{+} \mathrm{Na}^{+}-1,286.7\right], 233.8$. IR (KBr) $v_{\max } / \mathrm{cm}^{-1}: 3352,3087,3050,2967,2261$, 1622, 1580, 1476, 1300, 1239, 1148, 1018, 753. Anal. Calc. for $\mathrm{C}_{14} \mathrm{H}_{12} \mathrm{~N}_{6}: \mathrm{C}, 63.64 ; \mathrm{H}, 4.54 ; \mathrm{N}, 31.82$. Found: C, 63.51; H, 4.59; N, $31.93 \%$. 
9- $\beta$-cyanoethyl-6-(p-tolylamino) purine $(4 c)$. Brokenwhite powder; mp $173-174{ }^{\circ} \mathrm{C} .{ }^{1} \mathrm{H}$ NMR (DMSO- $d_{6}$ ) $\delta$ $2.284\left(\mathrm{~s}, 3 \mathrm{H}, \mathrm{CH}_{3}\right), 3.218\left(\mathrm{t}, 2 \mathrm{H}, J 6.4 \mathrm{~Hz}, \mathrm{CH}_{2} \mathrm{CH}_{2} \mathrm{CN}\right)$, $4.521\left(\mathrm{t}, 2 \mathrm{H}, J 6.4 \mathrm{~Hz}, \mathrm{NCH}_{2} \mathrm{CH}_{2}\right), 7.141(\mathrm{~d}, 2 \mathrm{H}, J 8.4 \mathrm{~Hz}$, $\left.\mathrm{H}_{\mathrm{Ar}}\right), 7.816\left(\mathrm{~d}, 2 \mathrm{H}, J 8.4 \mathrm{~Hz}, \mathrm{H}_{\mathrm{Ar}}\right), 8.364(\mathrm{~s}, 1 \mathrm{H}, \mathrm{H}-2), 8.403$ (s, 1H, H-8), 9.811 (s, 1H, NH). ${ }^{13} \mathrm{C}$ NMR (DMSO- $\left.d_{6}\right) \delta$ $18.60\left(\mathrm{CH}_{2} \mathrm{CN}\right), 20.91\left(\mathrm{CH}_{3}\right), 39.42\left(\mathrm{NCH}_{2} \mathrm{CH}_{2}\right), 118.71$ $(\mathrm{CN}), 120.07$ (C-5), 121.42, 129.23, 132.06, $137.46\left(\mathrm{C}_{\mathrm{Ar}}\right)$, 141.78 (C-8), 149.93 (C-6), 151.23 (C-4), 152.56 (C-2). MS (ESI) $m / z$ [ $\left.\mathrm{M}^{+} \mathrm{Na}^{+}-1,300.7\right], 145.9 . \mathrm{IR}(\mathrm{KBr}) \mathrm{v}_{\max } /$ $\mathrm{cm}^{-1}$ : 3386, 3089, 2919, 2860, 2249, 1616, 1585, 1476, 1367, 821. Anal. Calc. for $\mathrm{C}_{15} \mathrm{H}_{14} \mathrm{~N}_{6}: \mathrm{C}, 64.75 ; \mathrm{H}, 5.04 ; \mathrm{N}$, 30.22. Found: C, 64.49; H, 5.21; N, 30.35\%.

9- $\beta$-cyanoethyl-6-(p-methoxyphenylamino) purine $(\mathbf{4 d})$. Colorless column crystal; mp 80-82 ${ }^{\circ} \mathrm{C}$. ${ }^{1} \mathrm{H}$ NMR (DMSO$\left.d_{6}\right) \delta 3.216\left(\mathrm{t}, 2 \mathrm{H}, J 6.4 \mathrm{~Hz}, \mathrm{CH}_{2} \mathrm{CH}_{2} \mathrm{CN}\right), 3.750(\mathrm{~s}, 3 \mathrm{H}$, $\left.\mathrm{OCH}_{3}\right), 4.517$ (t, 2H, J 6.4Hz, $\left.\mathrm{NCH}_{2} \mathrm{CH}_{2}\right), 6.924$ (d, 2H, J $\left.8.8 \mathrm{~Hz}, \mathrm{H}_{\mathrm{Ar}}\right), 7.794\left(\mathrm{~d}, 2 \mathrm{H}, J 9.2 \mathrm{~Hz}, \mathrm{H}_{\mathrm{Ar}}\right), 8.344(\mathrm{~s}, 1 \mathrm{H}, \mathrm{H}-$ 2), 8.370 (s, 1H, H-8), 9.765 (s, $1 \mathrm{H}, \mathrm{NH}) .{ }^{13} \mathrm{C} \mathrm{NMR}$ $\left(\mathrm{DMSO}-d_{6}\right) \delta 18.62\left(\mathrm{CH}_{2} \mathrm{CN}\right), 39.41\left(\mathrm{NCH}_{2} \mathrm{CH}_{2}\right), 55.64$ $\left(\mathrm{OCH}_{3}\right), 118.72(\mathrm{CN}), 119.91(\mathrm{C}-5), 114.06,123.23$, 132.95, $152.63\left(\mathrm{C}_{\mathrm{Ar}}\right), 141.61(\mathrm{C}-8), 149.82(\mathrm{C}-6), 152.67$ (C-4), 155.61 (C-2). MS (ESI) $m / z$ [ $\left.\mathrm{M}^{+} \mathrm{Na}^{+}-1,316.7\right]$, 263.7. IR (KBr) $v_{\max } / \mathrm{cm}^{-1}: 3466,3301,3204,3101,2978$, 2881, 2253, 1620, 1587, 1512, 1471, 1294, 1035, 797. Anal. Calc. for $\mathrm{C}_{15} \mathrm{H}_{14} \mathrm{~N}_{6} \mathrm{O}$ : C, 61.22; H, 4.76; N, 28.57 . Found: C, 61.09; H, 4.84; N, $28.64 \%$.

9- $\beta$-cyanoethyl-6-(p-ethoxyphenylamino) purine (4e). Gray flakes; mp 146-148 ${ }^{\circ} \mathrm{C} .{ }^{1} \mathrm{H}$ NMR (DMSO- $\left.d_{6}\right) \delta 1.328$ (t, $3 \mathrm{H}$, $\left.J 7.2 \mathrm{~Hz}, \mathrm{CH}_{2} \mathrm{CH}_{3}\right), 3.215$ (t, 2H, J 6.4Hz, $\left.\mathrm{CH}_{2} \mathrm{CH}_{2} \mathrm{CN}\right), 4.009$ $\left(\mathrm{q}, 2 \mathrm{H}, J 7.2 \mathrm{~Hz}, \mathrm{OCH}_{2} \mathrm{CH}_{3}\right), 4.513(\mathrm{t}, 2 \mathrm{H}, J 6.4 \mathrm{~Hz}$, $\left.\mathrm{NCH}_{2} \mathrm{CH}_{2}\right), 6.907$ (d, 2H, J 9.2Hz, $\left.\mathrm{H}_{\mathrm{Ar}}\right), 7.783(\mathrm{~d}, 2 \mathrm{H}, J$ $8.8 \mathrm{~Hz}, \mathrm{H}_{\mathrm{Ar}}$ ), 8.343 (s, 1H, H-2), 8.365 (s, 1H, H-8), 9.758 (s, $1 \mathrm{H}, \mathrm{NH}) .{ }^{13} \mathrm{C} \mathrm{NMR}\left(\mathrm{DMSO}-d_{6}\right) \delta 15.16\left(\mathrm{OCH}_{2} \mathrm{CH}_{3}\right), 18.60$ $\left(\mathrm{CH}_{2} \mathrm{CN}\right), 39.42\left(\mathrm{NCH}_{2} \mathrm{CH}_{2}\right), 118.72(\mathrm{CN}), 119.90(\mathrm{C}-5)$, 114.60, 123.15, 132.88, $152.62\left(\mathrm{C}_{\mathrm{Ar}}\right), 141.61(\mathrm{C}-8), 149.81$ (C-6), 154.84 (C-4), 158.64 (C-2). MS (ESI) $m / z$ [ $\mathrm{M}^{+} \mathrm{Na}^{+}-1$, 330.8], 308.8. IR (KBr) $v_{\max } / \mathrm{cm}^{-1}: 3309,3231,3107,2979$, 1930, 2881, 2252, 1618, 1587, 1512, 1479, 1299, 1048, 840. Anal. Calc. for $\mathrm{C}_{16} \mathrm{H}_{16} \mathrm{~N}_{6} \mathrm{O}: \mathrm{C}, 62.34 ; \mathrm{H}, 5.19 ; \mathrm{N}, 27.27$. Found: C, 62.11; H, 5.31; N, $27.46 \%$.

2-chloro-9- $\beta$-cyanoethyl-6-cyclohexylamino purine (5a). White powder; mp 179-181 ${ }^{\circ} \mathrm{C} .{ }^{1} \mathrm{H}$ NMR (DMSO- $\left.d_{6}\right) \delta$ 1.107-1.923 (m, 10H, H cyclohexyl), 3.135 (t, 2H, J 6.4Hz, $\mathrm{CH}_{2} \mathrm{CH}_{2} \mathrm{CN}$ ), 4.005 (br, 1H, H cyclohexyl), 4.411 (t, 2H, J $6.4 \mathrm{~Hz}, \mathrm{NCH}_{2} \mathrm{CH}_{2}$ ), 8.163 (d, 1H, J 8.0Hz, NH), 8.199 (s, 1H, H-8). ${ }^{13} \mathrm{C}$ NMR (DMSO- $\left.d_{6}\right) \delta 18.62\left(\mathrm{CH}_{2} \mathrm{CN}\right), 25.13$, 25.36, 25.56, 32.47, 33.48, 49.45 (C cyclohexy), 38.76
$\left(\mathrm{NCH}_{2} \mathrm{CH}_{2}\right), 118.38(\mathrm{C}-5), 118.65(\mathrm{CN}), 141.15$ (C-8), 150.13 (C-6), 153.79 (C-4), 154.66 (C-2). MS (ESI) $m / z\left[\mathrm{M}^{+} \mathrm{Na}^{+}-1,326.7\right], 233.8$. Anal. Calc. for $\mathrm{C}_{14} \mathrm{H}_{17} \mathrm{ClN}_{6}$ : C, 55.17; H, 5.62; N, 27.57. Found: C, 54.97; H, 5.69; N, $27.73 \%$.

2-chloro-9- $\beta$-cyanoethyl-6-phenylamino purine (5b). White powder; mp 266-268 ${ }^{\circ} \mathrm{C}$. ${ }^{1} \mathrm{H}$ NMR (DMSO- $\left.d_{6}\right) \delta 3.187(\mathrm{t}$, $\left.2 \mathrm{H}, J 6.4 \mathrm{~Hz}, \mathrm{CH}_{2} \mathrm{CH}_{2} \mathrm{CN}\right), 4.492(\mathrm{t}, 2 \mathrm{H}, J 6.4 \mathrm{~Hz}$, $\left.\mathrm{NCH}_{2} \mathrm{CH}_{2}\right), 7.113\left(\mathrm{t}, 1 \mathrm{H}, J 7.2 \mathrm{~Hz}, \mathrm{H}_{\mathrm{Ar}}\right), 7.374(\mathrm{t}, 2 \mathrm{H}, J$ $\left.7.6 \mathrm{~Hz}, \mathrm{H}_{\mathrm{Ar}}\right), 7.845\left(\mathrm{~d}, 2 \mathrm{H}, J 8.0 \mathrm{~Hz}, \mathrm{H}_{\mathrm{Ar}}\right), 8.388(\mathrm{~s}, 1 \mathrm{H}, \mathrm{H}-$ 8), 10.348 (s, 1H, NH). ${ }^{13} \mathrm{C} \mathrm{NMR}\left(\mathrm{DMSO}-d_{6}\right) \delta 18.64$ $\left(\mathrm{CH}_{2} \mathrm{CN}\right), 39.94\left(\mathrm{NCH}_{2} \mathrm{CH}_{2}\right), 118.65(\mathrm{CN}), 119.20(\mathrm{C}-5)$, 121.81, 124.03, 128.97, $139.14\left(\mathrm{C}_{\mathrm{Ar}}\right), 142.49(\mathrm{C}-8), 149.32$ (C-6), 151.15 (C-4), 152.91 (C-2). MS (ESI) $\mathrm{m} / z$ [ $\mathrm{M}^{+} \mathrm{Na}^{+}-$ 1, 320.7], 298.8, 256.9, 101.9, 88.0. IR (KBr) $v_{\max } / \mathrm{cm}^{-1}$ : 3346, 3087, 3062, 2973, 2935, 2258, 1622, 1578, 1500, 1452, 1318, 1284, 756. Anal. Calc. for $\mathrm{C}_{14} \mathrm{H}_{11} \mathrm{ClN}_{6}$ : C, 56.28; H, 3.69; N, 28.14. Found: C, 55.97; H, 3.81; N, $28.36 \%$.

2-chloro-9- $\beta$-cyanoethyl-6-(p-tolylamino) purine $(5 \mathrm{c})$. Lustrous needle crystal; mp 254-256 ${ }^{\circ} \mathrm{C} .{ }^{1} \mathrm{H}$ NMR (DMSO$\left.d_{6}\right) \delta 2.297\left(\mathrm{~s}, 3 \mathrm{H}, \mathrm{CH}_{3}\right), 3.180\left(\mathrm{t}, 2 \mathrm{H}, \mathrm{J} 6.4 \mathrm{~Hz}, \mathrm{CH}_{2} \mathrm{CH}_{2} \mathrm{CN}\right)$, 4.480 (t, $\left.2 \mathrm{H}, J 6.4 \mathrm{~Hz}, \mathrm{NCH}_{2} \mathrm{CH}_{2}\right), 7.174(\mathrm{~d}, 2 \mathrm{H}, J 8.0 \mathrm{~Hz}$, $\left.\mathrm{H}_{\mathrm{Ar}}\right), 7.690\left(\mathrm{~d}, 2 \mathrm{H}, J 8.4 \mathrm{~Hz}, \mathrm{H}_{\mathrm{Ar}}\right), 8.363(\mathrm{~s}, 1 \mathrm{H}, \mathrm{H}-8), 10.256$ $(\mathrm{s}, 1 \mathrm{H}, \mathrm{NH}) .{ }^{13} \mathrm{C} \mathrm{NMR}\left(\mathrm{DMSO}-d_{6}\right) \delta 18.63\left(\mathrm{CH}_{2} \mathrm{CN}\right), 20.94$ $\left(\mathrm{CH}_{3}\right), 39.99\left(\mathrm{NCH}_{2} \mathrm{CH}_{2}\right), 118.65(\mathrm{CN}), 119.08(\mathrm{C}-5)$, 121.99, 129.39, 133.18, $136.51\left(\mathrm{C}_{\mathrm{Ar}}\right), 142.32(\mathrm{C}-8), 149.54$ (C-6), 151.04 (C-4), 152.98 (C-2). MS (ESI) m/z [ $\left[\mathrm{M}^{+} \mathrm{Na}^{+}-\right.$ 1, 334.7], 271.9, 227.9, 145.8, 96.9. IR (KBr) $v_{\max } / \mathrm{cm}^{-1}$ : 3339, 3055, 2930, 2264, 1623, 1579, 1514, 1456, 1252, 818. Anal. Calc. for $\mathrm{C}_{15} \mathrm{H}_{13} \mathrm{ClN}_{6}$ : C, 57.60; H, 4.16; N, 26.88. Found: C, 57.51; H, 4.22; N, 26.93\%.

2-chloro-9- $\beta$-cyanoethyl-6-(p-methoxyphenylamino) purine $(\mathbf{5 d})$. Lustrous flakes; mp $239-240{ }^{\circ} \mathrm{C}$. ${ }^{1} \mathrm{H}$ NMR $\left(\mathrm{DMSO}-d_{6}\right) \delta 3.178\left(\mathrm{t}, 2 \mathrm{H}, J 6.4 \mathrm{~Hz}, \mathrm{CH}_{2} \mathrm{CH}_{2} \mathrm{CN}\right), 3.764$ $\left(\mathrm{s}, 3 \mathrm{H}, \mathrm{OCH}_{3}\right), 4.476\left(\mathrm{t}, 2 \mathrm{H}, J 6.4 \mathrm{~Hz}, \mathrm{NCH}_{2} \mathrm{CH}_{2}\right), 6.954$ $\left(\mathrm{d}, 2 \mathrm{H}, J 8.8 \mathrm{~Hz}, \mathrm{H}_{\mathrm{Ar}}\right), 7.691\left(\mathrm{~d}, 2 \mathrm{H}, J 8.8 \mathrm{~Hz}, \mathrm{H}_{\mathrm{Ar}}\right), 8.343$ (s, 1H, H-8), 10.205 (s, 1H, NH). $\left.{ }^{13} \mathrm{C} \mathrm{NMR} \mathrm{(DMSO-} d_{6}\right) \delta$ $18.65\left(\mathrm{CH}_{2} \mathrm{CN}\right), 39.49\left(\mathrm{NCH}_{2} \mathrm{CH}_{2}\right), 55.68\left(\mathrm{OCH}_{3}\right), 118.65$ $(\mathrm{CN}), 118.94$ (C-5), 114.20, 123.71, 131.96, $150.91\left(\mathrm{C}_{\mathrm{Ar}}\right)$, 142.15 (C-8), 153.06 (C-6), 153.12 (C-4), 156.23 (C-2). MS (ESI) $m / z\left[\mathrm{M}^{+} \mathrm{Na}^{+}-1,350.7\right], 328.8,292.8,239.8$, 224.7, 196.7. IR (KBr) $v_{\max } / \mathrm{cm}^{-1}: 3337,3063,2962,2935$, 2835, 2258, 1624, 1589, 1512, 1478, 1309, 1248, 1048, 827. Anal. Calc. for $\mathrm{C}_{15} \mathrm{H}_{13} \mathrm{ClN}_{6} \mathrm{O}: \mathrm{C}, 54.79 ; \mathrm{H}, 3.96 ; \mathrm{N}$, 25.57. Found: C, 54.66; H, 4.03; N, $25.64 \%$.

2-amino-9- $\beta$-cyanoethyl-6-cyclohexylamino purine (6a). White powder; mp $135-136{ }^{\circ} \mathrm{C} .{ }^{1} \mathrm{H}$ NMR (DMSO- $d_{6}$ ) $\delta$ 
1.085-1.861 (m, 10H, H cyclohexyl), 3.088 (t, $2 \mathrm{H}, J 6.4 \mathrm{~Hz}$, $\mathrm{CH}_{2} \mathrm{CH}_{2} \mathrm{CN}$ ), 4.060 (br, 1H, H cyclohexyl), 4.243 (t, 2H, $J$ 6.4Hz, $\left.\mathrm{NCH}_{2} \mathrm{CH}_{2}\right), 5.846\left(\mathrm{~s}, 2 \mathrm{H}, \mathrm{NH}_{2}\right), 6.914(\mathrm{~s}, 1 \mathrm{H}$, $\mathrm{NH}), 7.742(\mathrm{~s}, 1 \mathrm{H}, \mathrm{H}-8) .{ }^{13} \mathrm{C}$ NMR (DMSO- $\left.d_{6}\right) \delta 18.34$ $\left(\mathrm{CH}_{2} \mathrm{CN}\right), 25.51,25.69,33.13,48.54$ (C cyclohexy), 38.76 $\left(\mathrm{NCH}_{2} \mathrm{CH}_{2}\right), 113.48(\mathrm{C}-5), 118.85(\mathrm{CN}), 137.04(\mathrm{C}-8)$, 152.31 (C-6), 154.63 (C-4), 160.76 (C-2). MS (ESI) m/z $\left[\mathrm{M}^{+} \mathrm{Na}^{+}-1,307.8\right], 285.9,254.8,203.7,150.8$. IR (KBr) $v_{\max } / \mathrm{cm}^{-1}: 3331,3218,3103,2929,2854,2246,1637$, $1602,1486,1399,791$. Anal. Calc. for $\mathrm{C}_{14} \mathrm{H}_{19} \mathrm{~N}_{7}$ : C, 58.95; H, 6.67; N, 34.39. Found: C, 58.82; H, 6.79; N, $34.45 \%$.

2-amino-9- $\beta$-cyanoethyl-6-phenylamino purine $(\boldsymbol{6} \boldsymbol{b})$. Colorless flake crystal; mp $246-247^{\circ} \mathrm{C}$. ${ }^{1} \mathrm{H}$ NMR (DMSO$\left.d_{6}\right) \delta 3.141\left(\mathrm{t}, 2 \mathrm{H}, J 6.4 \mathrm{~Hz}, \mathrm{CH}_{2} \mathrm{CH}_{2} \mathrm{CN}\right), 4.313(\mathrm{t}, 2 \mathrm{H}, J$ $6.4 \mathrm{~Hz}, \mathrm{NCH}_{2} \mathrm{CH}_{2}$ ), 6.204 (s, $\left.2 \mathrm{H}, \mathrm{NH}_{2}\right), 6.981$ (t, $1 \mathrm{H}, J$ $\left.7.2 \mathrm{~Hz}, \mathrm{H}_{\mathrm{Ar}}\right), 7.280\left(\mathrm{t}, 2 \mathrm{H}, J 8.0 \mathrm{~Hz}, \mathrm{H}_{\mathrm{Ar}}\right), 7.910(\mathrm{~s}, 1 \mathrm{H}, \mathrm{H}-$ 8), $8.017\left(\mathrm{~d}, 2 \mathrm{H}, J 8.0 \mathrm{~Hz}, \mathrm{H}_{\mathrm{Ar}}\right), 9.380(\mathrm{~s}, 1 \mathrm{H}, \mathrm{NH}) .{ }^{13} \mathrm{C}$ NMR $\left(\right.$ DMSO- $\left.d_{6}\right) \delta 18.36\left(\mathrm{CH}_{2} \mathrm{CN}\right), 38.87\left(\mathrm{NCH}_{2} \mathrm{CH}_{2}\right)$, $118.83(\mathrm{CN}), 120.65$ (C-5), 114.17, 122.25, 128.71, 138.13 $\left(\mathrm{C}_{\mathrm{Ar}}\right), 140.77(\mathrm{C}-8), 152.18(\mathrm{C}-6), 152.82(\mathrm{C}-4), 160.44$ (C-2). MS (ESI) $m / z$ [ $\left.\mathrm{M}^{+} \mathrm{Na}^{+}-1,301.6\right], 248.7$. IR (KBr) $v_{\max } / \mathrm{cm}^{-1}: 3445,3391,3329,3202,3099,2984,2935$, $2252,1645,1617,1579,1498,1439,787$. Anal. Calc. for $\mathrm{C}_{14} \mathrm{H}_{13} \mathrm{~N}_{7}: \mathrm{C}, 60.22 ; \mathrm{H}, 4.66$; N, 35.13. Found: C, 60.13; $\mathrm{H}, 4.73 ; \mathrm{N}, 35.19 \%$.

2-amino-9- $\beta$-cyanoethyl-6-( $p$-tolylamino) purine $(\boldsymbol{6 c})$. White needle crystal; mp 208-209 ${ }^{\circ} \mathrm{C} .{ }^{1} \mathrm{H}$ NMR (DMSO$\left.d_{6}\right) \delta 2.269\left(\mathrm{~s}, 3 \mathrm{H}, \mathrm{CH}_{3}\right), 3.134\left(\mathrm{t}, 2 \mathrm{H}, J 6.4 \mathrm{~Hz}, \mathrm{CH}_{2} \mathrm{CH}_{2} \mathrm{CN}\right.$ ), 4.305 (t, $\left.2 \mathrm{H}, J 6.4 \mathrm{~Hz}, \mathrm{NCH}_{2} \mathrm{CH}_{2}\right), 6.153$ (s, $\left.2 \mathrm{H}, \mathrm{NH}_{2}\right), 7.083$ $\left(\mathrm{d}, 2 \mathrm{H}, J 8.4 \mathrm{~Hz}, \mathrm{H}_{\mathrm{Ar}}\right), 7.864\left(\mathrm{~d}, 2 \mathrm{H}, J 8.4 \mathrm{~Hz}, \mathrm{H}_{\mathrm{Ar}}\right), 7.889$ (s, $1 \mathrm{H}, \mathrm{H}-8), 9.271$ (s, $1 \mathrm{H}, \mathrm{NH}) .{ }^{13} \mathrm{C}$ NMR (DMSO- $d_{6}$ ) $\delta 18.36$ $\left(\mathrm{CH}_{2} \mathrm{CN}\right), 20.89\left(\mathrm{CH}_{3}\right), 38.86\left(\mathrm{NCH}_{2} \mathrm{CH}_{2}\right), 118.83(\mathrm{CN})$, 120.82 (C-5), 114.10, 129.14, 131.13, $137.98\left(\mathrm{C}_{\mathrm{Ar}}\right), 138.17$ (C-8), 152.06 (C-6), 152.87 (C-4), 160.46 (C-2). MS (ESI) $\mathrm{m} / \mathrm{z}\left[\mathrm{M}^{+} \mathrm{Na}^{+}-1,315.8\right], 293.8,245.9,228.8,198.7,156.8$, 144.7, 117.9, 82.0, 65.1. IR (KBr) $v_{\max } / \mathrm{cm}^{-1}: 3457,3337$, 3104, 2944, 2855, 2252, 1627, 1597, 1513, 1482, 1417, 825, 788. Anal. Calc. for $\mathrm{C}_{15} \mathrm{H}_{15} \mathrm{~N}_{7}: \mathrm{C}, 61.43 ; \mathrm{H}, 5.12 ; \mathrm{N}$, 33.45. Found: C, 61.36; H, 5.17; N, 33.48\%.

2-amino-9- $\beta$-cyanoethyl-6-(p-methoxyphenylamino) purine (6d). Colorless needle crystal; mp 174-176 ${ }^{\circ} \mathrm{C} .{ }^{1} \mathrm{H}$ NMR $\left(\mathrm{DMSO}-d_{6}\right) \delta 3.133$ (t, $2 \mathrm{H}, J 6.4 \mathrm{~Hz}, \mathrm{CH}_{2} \mathrm{CH}_{2} \mathrm{CN}$ ), 3.738 (s, $3 \mathrm{H}, \mathrm{OCH}_{3}$ ), 4.303 (t, $2 \mathrm{H}, J 6.4 \mathrm{~Hz}, \mathrm{NCH}_{2} \mathrm{CH}_{2}$ ), 6.116 (s, $\left.2 \mathrm{H}, \mathrm{NH}_{2}\right), 6.863\left(\mathrm{~d}, 2 \mathrm{H}, J 8.8 \mathrm{~Hz}, \mathrm{H}_{\mathrm{Ar}}\right), 7.856(\mathrm{~d}, 2 \mathrm{H}, J$ $\left.8.8 \mathrm{~Hz}, \mathrm{H}_{\mathrm{Ar}}\right), 7.877(\mathrm{~s}, 1 \mathrm{H}, \mathrm{H}-8), 9.249(\mathrm{~s}, 1 \mathrm{H}, \mathrm{NH}) .{ }^{13} \mathrm{C}$ NMR (DMSO- $\left.d_{6}\right) \delta 18.37\left(\mathrm{CH}_{2} \mathrm{CN}\right), 38.86\left(\mathrm{NCH}_{2} \mathrm{CH}_{2}\right)$, $55.61\left(\mathrm{OCH}_{3}\right), 118.84(\mathrm{CN}), 120.15(\mathrm{C}-5), 113.95,122.46$, 133.82, 137.86, $152.92\left(\mathrm{C}_{\mathrm{Ar}}\right), 141.59$ (C-8), 151.96 (C-6),
154.98 (C-4), 160.50 (C-2). MS (ESI) $m / z$ [ $\left.\mathrm{M}^{+} \mathrm{Na}^{+}-1,331.7\right]$, 309.8, 292.8, 267.8, 252.7, 224.7, 214.7, 199.7, 160.8, 92.4 . IR (KBr) $v_{\max } / \mathrm{cm}^{-1}: 3483,3327,3203,3008,2946,2848$, 2250, 1596, 1514, 1486, 1243, 1031, 786. Anal. Calc. for $\mathrm{C}_{15} \mathrm{H}_{15} \mathrm{~N}_{7} \mathrm{O}: \mathrm{C}, 58.25 ; \mathrm{H}, 4.85 ; \mathrm{N}, 31.72$. Found: C, 58.16; $\mathrm{H}, 4.78 ; \mathrm{N}, 31.91 \%$.

2-amino-9- $\beta$-cyanoethyl-6-(p-ethoxyphenylamino) purine (6e). White needle crystal; mp 177-178 ${ }^{\circ} \mathrm{C} .{ }^{1} \mathrm{H}$ NMR $\left(\right.$ DMSO- $\left.d_{6}\right) \delta 1.324$ (t, 3H, J 6.8Hz, $\mathrm{OCH}_{2} \mathrm{CH}_{3}$ ), 3.131 (t, $\left.2 \mathrm{H}, J 6.4 \mathrm{~Hz}, \mathrm{CH}_{2} \mathrm{CH}_{2} \mathrm{CN}\right), 3.998(\mathrm{q}, 2 \mathrm{H}, J 6.8 \mathrm{~Hz}$, $\mathrm{OCH}_{2} \mathrm{CH}_{3}$ ), 4.301 (t, $2 \mathrm{H}, J 6.4 \mathrm{~Hz}, \mathrm{NCH}_{2} \mathrm{CH}_{2}$ ), 6.114 (s, $\left.2 \mathrm{H}, \mathrm{NH}_{2}\right), 6.846\left(\mathrm{~d}, 2 \mathrm{H}, J 8.8 \mathrm{~Hz}, \mathrm{H}_{\mathrm{Ar}}\right), 7.851(\mathrm{~d}, 2 \mathrm{H}, J$ $\left.9.2 \mathrm{~Hz}, \mathrm{H}_{\mathrm{Ar}}\right), 7.876$ (s, $\left.1 \mathrm{H}, \mathrm{H}-8\right), 9.243$ (s, $\left.1 \mathrm{H}, \mathrm{NH}\right) .{ }^{13} \mathrm{C}$ NMR (DMSO- $\left.d_{6}\right) \delta 15.20\left(\mathrm{OCH}_{2} \mathrm{CH}_{3}\right), 18.60\left(\mathrm{CH}_{2} \mathrm{CN}\right)$, $38.86\left(\mathrm{NCH}_{2} \mathrm{CH}_{2}\right), 63.52\left(\mathrm{OCH}_{2} \mathrm{CH}_{3}\right), 118.84(\mathrm{CN}), 120.15$ (C-5), 113.97, 114.52, 122.41, 133.73, 137.85, $152.89\left(\mathrm{C}_{\mathrm{Ar}}\right)$, 141.59 (C-8), 151.93 (C-6), 154.23 (C-4), 160.47 (C-2). MS (ESI) $m / z$ [ $\left.\mathrm{M}^{+} \mathrm{Na}^{+}-1,323.8\right], 281.8,253.7,224.7,200.7$, $146.8,119.8,80.9$. IR (KBr) $v_{\max } / \mathrm{cm}^{-1}: 3487,3322,3195$, 2975, 2930, 2884, 2248, 1599, 1512, 1456, 1418, 1235, 1053, 832, 786. Anal. Calc. for $\mathrm{C}_{16} \mathrm{H}_{17} \mathrm{~N}_{7} \mathrm{O}: \mathrm{C}, 59.44 ; \mathrm{H}$, 5.26; N, 30.34. Found: C, 59.35; H, 5.29; N, $30.42 \%$.

6-(p-ethoxyphenylamino)-9-( $\beta$-D-ribofuranosyl) purine (7e). White powder; mp 191-192 ${ }^{\circ} \mathrm{C} .{ }^{1} \mathrm{H} N M R$ (DMSO- $d_{6}$ ) $\delta 1.330$ (t, $3 \mathrm{H}, J 7.2 \mathrm{~Hz}, \mathrm{CH}_{2} \mathrm{CH}_{3}$ ), 3.650 (dd, $\left.2 \mathrm{H}, J 12 \mathrm{~Hz}, \mathrm{H}-5^{\prime}\right)$, 3.898 (m, 1H, H-4'), 4.012 (q, 2H, J7.2Hz, $\mathrm{OCH}_{2} \mathrm{CH}_{3}$ ), 4.183 (m, 1H, H-3'), 4.648 (m, 1H, H-2'), 5.953 (d, 1H, J 7.0Hz, H-1'), 6.908 (d, 2H, $\left.J 8.8 \mathrm{~Hz}, \mathrm{H}_{\mathrm{Ar}}\right), 7.777(\mathrm{~d}, 2 \mathrm{H}, J 8.8 \mathrm{~Hz}$, $\mathrm{H}_{\mathrm{Ar}}$ ), 8.341 (s, 1H, H-2), 8.504 (s, 1H, H-8), 9.792 (s, 1H, $\mathrm{NH}) .{ }^{13} \mathrm{C}$ NMR (DMSO- $\left.d_{6}\right) \delta 15.16\left(\mathrm{CH}_{2} \mathrm{CH}_{3}\right), 62.05\left(5^{\prime}-\mathrm{C}\right)$, $63.57\left(\mathrm{CH}_{2} \mathrm{CH}_{3}\right), 71.03$ (3'-C), $74.03\left(2^{\prime}-\mathrm{C}\right), 86.32\left(4^{\prime}-\mathrm{C}\right)$, 88.35 (1'-C), 117.41 (5-C), 114.61, 115.77, 120.56, 123.21, 132.78, $149.53\left(\mathrm{C}_{\mathrm{Ar}}\right), 140.85(8-\mathrm{C}), 152.45(6-\mathrm{C}), 152.75$ (4C), 154.89 (2-C). MS (ESI) $m / z$ [ $\left.\mathrm{M}^{+} \mathrm{Na}^{+}-1,409.7\right], 387.8$, 362.0, 318.0, 274.0, 255.8, 227.7, 199.8, 171.7, 134.8, 119.9, 108.9. IR (KBr) $v_{\max } / \mathrm{cm}^{-1}: 3338,3223,3151,2983,2930$, 2871, 1645, 1596, 1512, 1478, 1240, 1058, 826, 791. Anal. Calc. for $\mathrm{C}_{18} \mathrm{H}_{21} \mathrm{~N}_{5} \mathrm{O}_{5}: \mathrm{C}, 55.81 ; \mathrm{H}, 5.43 ; \mathrm{N}, 18.09$. Found: $\mathrm{C}$, $55.72 ; \mathrm{H}, 5.51 ; \mathrm{N}, 18.13 \%$.

2-chloro-6-cyclohexylamino-9-[(2-hydroxylethoxy) methyl]purine $(\mathbf{9 a})$. White powder; mp $165-167{ }^{\circ} \mathrm{C} .{ }^{1} \mathrm{H}$ NMR (DMSO- $\left.d_{6}\right) \delta 1.107-1.922$ (m, 10H, H cyclohexyl), 3.493 (m, 4H, $\mathrm{HOCH}_{2} \mathrm{CH}_{2} \mathrm{O}$ ), 4.004 (br, $1 \mathrm{H}, \mathrm{H}$ cyclohexyl), $5.518\left(\mathrm{~s}, 2 \mathrm{H}, \mathrm{NCH}_{2} \mathrm{O}\right), 8.154$ (d, $\left.1 \mathrm{H}, J 7.2 \mathrm{~Hz}, \mathrm{NH}\right), 8.283$ (s, 1H, H-8). ${ }^{13} \mathrm{C}$ NMR (DMSO- $\left.d_{6}\right) \delta 33.48,32.47,25.56$, 25.35 (C cyclohexy), $49.47(\mathrm{NCH}), 60.34\left(\mathrm{HOCH}_{2} \mathrm{CH}_{2} \mathrm{O}\right)$, $71.23\left(\mathrm{HOCH}_{2} \mathrm{CH}_{2} \mathrm{O}\right), 72.92\left(\mathrm{NCH}_{2} \mathrm{O}\right), 118.33(5-\mathrm{C})$, 141.77 (8-C), 150.41 (6-C), 154.08 (4-C), 154.70 (2-C). 
MS (ESI) $m / z$ [ $\mathrm{M}^{+} \mathrm{Na}^{+}-1$ 347.7], 325.8, 311.6, 284.0, 228.8, 101.9. IR (KBr) $v_{\max } / \mathrm{cm}^{-1}: 3441,3236,2937,2923,2857$, 1621, 1310, 1216. Anal. Calc. for $\mathrm{C}_{14} \mathrm{H}_{20} \mathrm{ClN}_{5} \mathrm{O}_{2}$ : C, 51.61; H, 6.14; N, 21.51. Found: C, 51.47; H, 6.18; N, $21.63 \%$.

\section{Acknowledgments}

We thank the National Natural Science Foundation of China (No: 20372018) for financial support.

\section{Supplementary Information}

General procedure, characterization of all compounds and ${ }^{1} \mathrm{H}$ NMR, ${ }^{13} \mathrm{C}$ NMR and IR of selected compounds. Supplementary data are available free of charge as PDF file at http://jbcs.sbq.org.br

\section{References}

1. Dalpozzo, R.; De Nino, A.; Maiuolo, L.; Procopio, A.; De Munno, G.; Sindona, G.; Tetrahedron 2001, 57, 4035.

2. Bisacchi, G. S.; Singh, J.; Godfrey, J. D.; Kissick, J. T. P.; Mitt, T.; Malley, M. F.; Marco, J. D. D.; Gougoutas, J. Z.; Mueller, R. H.; Zahler, R.; J. Org. Chem. 1995, 60, 2902.

3. Cappuccino, J. G.; George, M.; Merker, P. C; Tarnowski, G. S.; Cancer Res. 1964, 24, 1243.

4. Tret'yakova, G. S.; Nedel'kina, N. N.; Cherkasov, V. M.; Ukrainskii Khimicheskii Zhurnal 1972, 38, 602.

5. Florence, B.-P.; Haesslein; Jean-Luc.; PCT Int. Appl. WO 2002051843 A1 20020704, 2002.

6. Merckle GmbH.; Eur. Pat. Appl. EP 1444982 A1 20040811, 2004.

7. Kwatra, M. M.; Leung, E.; Hosey, M. M.; Green, R. D.; J. Med. Chem. 1987, 30, 954; Vittori, S.; Lorenzen, A.; Stannek, C.; Costanzi, S.; Volpini, R.; IJzerman, A. P.; Kunzel, J. K. V. F. D.; Cristalli, G.; J. Med. Chem. 2000, 43, 250; Moos, W. H.; Szotek, D. S.; Bruns, R. F.; J. Med. Chem. 1985, 28, 1383.

8. Altmann, E.; Cowan-Jacob, S. W; Missbach, M.; J. Med. Chem. 2004, 47, 5833
9. Kikugawa, K.; Iizuka, K.; Ichino, M.; J. Med. Chem. 1973, 16, 358.

10. Knutsen, L. J. S.; Lau, J.; Petersen, H.; Thomsen, C.; Weis, J. U.; Shalmi, M.; Judge, M. E.; Hansen, A. J.; Sheardown, M. J.; J. Med. Chem. 1999, 42, 3463.

11. Lanver, A.; Schmalz, H.-G.; Molecules 2005, 10, 508.

12. Elion, G. B.; Burgi, E.; Hitchings, G. H.; J. Am. Chem. Soc. 1952, 74, 411.

13. Girgis, N. S.; Pedersen, E. B.; Synthesis 1982, 6, 480; Fu, R.; Xu, X.; Dang, Q.; Bai, X.; J. Org. Chem. 2005, 70, 10810.

14. Wu, T. Y. H.; Schultz, P. G.; Ding, S.; Org. Lett. 2003, 5, 3587.

15. Fiorini, M. T.; Abell, C.; Tetrahedron Lett. 1998, 39, 1827.

16. Ran, C.; Dai, Q.; Harvey, R. G.; J. Org. Chem. 2005, 70, 3724.

17. Khalafi-Nezhad, A.; Rad, M. N. S.; Hakimelahi, G. H.; Helv. Chim. Acta. 2003, 86, 2396; Varma, R. S.; Green Chem. 1999 1, 43; Kappe, C. O.; Angew. Chem., Int. Ed. 2004, 43, 6250; Perreux, L.; Loupy, A.; Tetrahedron 2001, 57, 9199; Lidström, P.; Tierney, J.; Wathey, B.; Westman, J.; Tetrahedron 2001, 57, 9225.

18. Qu, G.-R.; Li, Y.; Han, H.-S.; J. Chem. Res. 2005, 167; Qu, G.R.; Liu, Q.-B.; Indian. J. Chem. B 2005, 44, 196.

19. Bai, L.; Wang, J.-X.; Zhang, Y.-M.; Green Chem. 2003, 5, 615.

20. Tundo, P.; Ananstas, P. T. In Green Chemistry: Challenging Perspectives, Oxford University Press: Oxford, 1999; Fringuelli, F.; Piermatti, O.; Pizzo, F.; Vaccaro, L.; Eur. J. Org. Chem. 2001, 439.

21. Fringuelli, F.; Pizzo, F.; Tortoioli, S.; Vaccaro, L.; Green Chem. 2003, 5, 436

22. Lira, E. P.; Huffman, C. W.; J. Org. Chem. 1966, 31, 2188; Baker, B. R.; Tanna, P. M.; J. Org. Chem. 1965, 30, 2857; Morris, J. R.; Peter, W. H.; Can. J. Chem. 1982, 60, 547.

23. Chem. Abstr. 1966, 65, 16970.

24. Okono, T.; Goya, S.; Takadate, A.; Eto, Y.; J. Pharm. Soc. Jpn. 1966, 86, 694 .

25. Hitchings, G. H.; Elion, G. B.; US 2691654, 1954.

26. Fleysher, M. H.; Bloch, A.; Hakala M. T.; Nichol, C. A.; J. Med. Chem. 1969, 12, 1056.

Received: October 30, 2005 Published on the web: June 29, 2006 


\title{
Microwave Assisted Synthesis of 6-Substituted Aminopurine Analogs in Water
}

\author{
Guirong Qu, ${ }^{*}$ Suhui Han, Zhiguang Zhang, Mingwei Geng and Feng Xue \\ College of Chemistry and Environmental Science, Henan Normal University, Xinxiang 453007, \\ Henan, P. R. China
}

General procedure for the synthesis of 6-substituted aminopurine analogs 1-7, a-e and $8 a, 8 a$ ', $9 a$

Amine (6 mmol) is added to a stirred suspension of 6chloropurine derivatives $\mathbf{1 - 9}(2 \mathrm{mmol})$ in water $(10 \mathrm{~mL})$ in a $50 \mathrm{~mL}$ round-bottomed flask. After vibration, the flask is moved into microwave oven and irradiated at $200 \mathrm{~W}$ for 10 minutes. At each interval, TLC monitors the reaction progress. When the reaction completed, the mixture is cooled to room temperature and desired 6-substituted aminopurine analogs 1-3,a-e and 7,a-e precipitate out. Then filter the solid directly followed by washing with cold water $(3 \times 5 \mathrm{~mL})$. The filtrate is concentrated to $1 / 3$ volume, and collects the products crystallized again from the mixture. The products 4-6,a-e and 8a, 8a', 9a are purified by column chromatography with $\mathrm{CHCl}_{3} / \mathrm{CH}_{3} \mathrm{OH}$ $(9 / 1, v / v)$ for 4-6,a-e and $\mathrm{CHCl}_{3} / \mathrm{CH}_{3} \mathrm{OH}(97 / 3, \mathrm{v} / \mathrm{v})$ for $\mathbf{8 a}, \mathbf{8} \mathbf{a}^{\prime}, 9 \mathbf{a}$ after the concentration of the reaction mixtures.

Physical data of the known compounds had been partly reported in the literatures (see references 23-27 in the original manuscript) and melting points of $\mathbf{2 a}$ and $\mathbf{3 a}$ are not identified with the literatures (see references 23 and 24 in the original manuscript) although the NMR spectra have proved their structures.

6-cyclohexylamino purine (1a)

White crystal; mp $210-211^{\circ} \mathrm{C}$ (lit. $210-211^{\circ} \mathrm{C}$ ). ${ }^{26}{ }^{1} \mathrm{H}$ NMR (DMSO- $\left.d_{6}\right) \delta 1.131-1.953$ (m, 10H, H cyclohexyl), 4.089 (s, 1H, H-1 cyclohexyl), 7.287 (d, 1H, J $8.4 \mathrm{~Hz}$, $\mathrm{NH}), 8.083$ (s, 1H, H-2), 8.162 (s, 1H, H-8).

6-phenylamino purine (1b)

White crystal; mp $279-282{ }^{\circ} \mathrm{C}$ (lit. $278-281{ }^{\circ} \mathrm{C}$ ). ${ }^{27}{ }^{1} \mathrm{H}$ NMR (DMSO- $\left.d_{6}\right) \delta 7.032\left(\mathrm{t}, 1 \mathrm{H}, J 7.2 \mathrm{~Hz}, \mathrm{H}_{\mathrm{Ar}}\right), 7.334$ (t, $\left.2 \mathrm{H}, J 8.0 \mathrm{~Hz}, \mathrm{H}_{\mathrm{Ar}}\right), 7.966\left(\mathrm{~d}, 2 \mathrm{H}, J 8.0 \mathrm{~Hz}, \mathrm{H}_{\mathrm{Ar}}\right), 8.307$ (s, 1H, H-2), 8.405 (s, 1H, H-8), 9.796 (s, 1H, NH). ${ }^{13} \mathrm{C} \mathrm{NMR}$

* e-mail: quguir@yahoo.com.cn $\left(\right.$ DMSO- $\left.d_{6}\right) \delta 118.83$ (5-C), 120.96, 122.86, 128.57, 128.86, 129.13, $129.44\left(\mathrm{C}_{\mathrm{Ar}}\right), 140.24(8-\mathrm{C}), 140.79$ (6C), 151.82 (4-C), 152.17 (2-C).

6-(p-tolylamino) purine (1c)

White needle crystal; mp 259-260 ${ }^{\circ} \mathrm{C}$ (lit. 242$243^{\circ} \mathrm{C}$ ) ${ }^{26}{ }^{1} \mathrm{H}$ NMR (DMSO- $\left.d_{6}\right) \delta 2.278\left(\mathrm{~s}, 3 \mathrm{H}, \mathrm{CH}_{3}\right.$ ), 7.134 $\left(\mathrm{d}, 2 \mathrm{H}, J 8.4 \mathrm{~Hz}, \mathrm{H}_{\mathrm{Ar}}\right), 7.820\left(\mathrm{~d}, 2 \mathrm{H}, J 8.4 \mathrm{~Hz}, \mathrm{H}_{\mathrm{Ar}}\right), 8.268$ (s, 1H, H-2), 8.359 (s, $1 \mathrm{H}, \mathrm{H}-8), 9.640$ (s, $1 \mathrm{H}, \mathrm{NH}) .{ }^{13} \mathrm{C}$ NMR (DMSO- $\left.d_{6}\right) \delta 20.90\left(\mathrm{CH}_{3}\right), 118.95(5-\mathrm{C}), 121.07$, 129.26, 131.77, $137.68\left(\mathrm{C}_{\mathrm{Ar}}\right), 140.53(8-\mathrm{C}), 148.46$ (6C), 151.95 (4-C), 152.26 (2-C).

6-(p-methoxyphenylamino) purine (1d)

Colorless needle crystal; mp 279-280 ${ }^{\circ} \mathrm{C} .{ }^{1} \mathrm{H}$ NMR $\left(\right.$ DMSO- $\left.d_{6}\right) \delta 3.750\left(\mathrm{~s}, 3 \mathrm{H}, \mathrm{OCH}_{3}\right), 6.916(\mathrm{~d}, 2 \mathrm{H}, J 8.8$ $\left.\mathrm{Hz}, \mathrm{H}_{\mathrm{Ar}}\right), 7.806\left(\mathrm{~d}, 2 \mathrm{H}, J 8.8 \mathrm{~Hz}, \mathrm{H}_{\mathrm{Ar}}\right), 8.232(\mathrm{~s}, 1 \mathrm{H}, \mathrm{H}-2)$, 8.307 (s, 1H, H-8), 9.603 (s, 1H, NH), 13.095 (br, 1H, H9). ${ }^{13} \mathrm{C}$ NMR (DMSO- $\left.d_{6}\right) \delta 55.65\left(\mathrm{OCH}_{3}\right), 119.66(5-\mathrm{C})$, 114.04, 122.92, $133.27\left(\mathrm{C}_{\mathrm{Ar}}\right), 139.96(8-\mathrm{C}), 150.64$ (6C), 152.37 (4-C), 155.38 (2-C).

6-(p-ethoxyphenylamino) purine (1e)

White needle crystal; mp 270-272 ${ }^{\circ} \mathrm{C} .{ }^{1} \mathrm{H}$ NMR (DMSO- $d_{6}$ ) $\delta 1.323$ (t, 3H, $J 6.8 \mathrm{~Hz}, \mathrm{CH}_{2} \mathrm{CH}_{3}$ ), 4.001 (q, $\left.2 \mathrm{H}, J 7.2 \mathrm{~Hz}, \mathrm{OCH}_{2} \mathrm{CH}_{3}\right), 6.899\left(\mathrm{~d}, 2 \mathrm{H}, J 9.2 \mathrm{~Hz}, \mathrm{H}_{\mathrm{Ar}}\right.$ ), 7.793 (d, 2H, J 9.2 Hz, $\mathrm{H}_{\mathrm{Ar}}$ ), 8.243 (s, 1H, H-2), 8.320 (s, $1 \mathrm{H}, \mathrm{H}-8), 9.578$ (s, 1H, NH), 13.090 (br, $1 \mathrm{H}, \mathrm{H}-9) .{ }^{13} \mathrm{C}$ NMR (DMSO- $\left.d_{6}\right) \delta 15.17\left(\mathrm{CH}_{2} \mathrm{CH}_{3}\right), 63.55\left(\mathrm{CH}_{2} \mathrm{CH}_{3}\right)$, $119.13(5-\mathrm{C}), 114.62,122.80,133.15\left(\mathrm{C}_{\mathrm{Ar}}\right), 140.20(8-$ C), 149.68 (6-C), 152.36 (4-C), 154.63 (2-C).

2-chloro-6-cyclohexylamino purine (2a)

White powder; mp 290-294 ${ }^{\circ} \mathrm{C} .{ }^{1} \mathrm{H}$ NMR (DMSO- $d_{6}$ ) $\delta$ 1.044-1.884 (m, 10H, H cyclohexyl), 3.978 (s, 1H, H-1 cyclohexyl), 7.885 (d, 1H, NH), 8.204 (s, 1H, H-8). 
2-chloro-6-phenylamino purine $(2 \boldsymbol{b})$

White needle crystal; mp $>300{ }^{\circ} \mathrm{C} .{ }^{1} \mathrm{H}$ NMR (DMSOd) $\delta 7.096\left(\mathrm{t}, 1 \mathrm{H}, J 7.6 \mathrm{~Hz}, \mathrm{H}_{\mathrm{Ar}}\right), 7.369(\mathrm{t}, 2 \mathrm{H}, J 7.6 \mathrm{~Hz}$, $\left.\mathrm{H}_{\mathrm{Ar}}\right), 7.848\left(\mathrm{~d}, 2 \mathrm{H}, J 7.6 \mathrm{~Hz}, \mathrm{H}_{\mathrm{Ar}}\right), 8.303(\mathrm{~s}, 1 \mathrm{H}, \mathrm{H}-8), 10.126$ (s, 1H, NH), 13.313 (bs, 1H, H-9). $\left.{ }^{13} \mathrm{C} \mathrm{NMR} \mathrm{(DMSO-} d_{6}\right) \delta$ 118.47 (5-C), 121.16, 123.74, 128.99, $139.37\left(\mathrm{C}_{\mathrm{Ar}}\right), 141.01$ (8-C), 151.68 (6-C), 152.02 (4-C), 152.57 (2-C).

\section{2-chloro-6-(p-tolylamino) purine $(2 c)$}

White powder; mp $>300{ }^{\circ} \mathrm{C} .{ }^{1} \mathrm{H}$ NMR (DMSO- $\left.d_{6}\right) \delta$ 2.294 (s, 3H, $\mathrm{CH}_{3}$ ), 7.169 (d, 2H, J $8.0 \mathrm{~Hz}, \mathrm{H}_{\mathrm{Ar}}$ ), 7.697 (d, $\left.2 \mathrm{H}, J 8.0 \mathrm{~Hz}, \mathrm{H}_{\mathrm{Ar}}\right), 8.276$ (s, 1H, H-8), 10.081 (s, 1H, $\mathrm{NH}), 13.272$ (br, 1H, H-9). ${ }^{13} \mathrm{C}$ NMR (DMSO- $\left.d_{6}\right) \delta 20.92$ $\left(\mathrm{CH}_{3}\right), 118.86$ (5-C), 121.67, 129.40, 132.85, $136.75\left(\mathrm{C}_{\mathrm{Ar}}\right)$, 140.84 (8-C), 149.56 (6-C), 151.97 (4-C), 152.66 (2-C).

2-chloro-6-(p-methoxyphenylamino) purine $(2 d)$

White powder; mp $>300{ }^{\circ} \mathrm{C} .{ }^{1} \mathrm{H}$ NMR (DMSO- $\left.d_{6}\right) \delta$ $3.761\left(\mathrm{~s}, 3 \mathrm{H}, \mathrm{OCH}_{3}\right), 6.950\left(\mathrm{~d}, 2 \mathrm{H}, J 8.8 \mathrm{~Hz}, \mathrm{H}_{\mathrm{Ar}}\right), 7.696$ (d, 2H, J $8.8 \mathrm{~Hz}, \mathrm{H}_{\mathrm{Ar}}$ ), 8.260 (s, 1H, H-8), 10.021 (s, 1H, $\mathrm{NH}), 13.224$ (br, 1H, H-9). ${ }^{13} \mathrm{C}$ NMR (DMSO- $d_{6}$ ) $\delta 55.67$ $\left(\mathrm{OCH}_{3}\right), 118.76(5-\mathrm{C}), 114.23,123.34,132.23\left(\mathrm{C}_{\mathrm{Ar}}\right)$, 140.95 (8-C), 149.43 (6-C), 152.76 (4-C), 156.04 (2-C).

2-amino-6-cyclohexylamino purine (3a)

Lustrous white flakes; mp 184-187 ${ }^{\circ} \mathrm{C} .{ }^{1} \mathrm{H}$ NMR $\left(\mathrm{DMSO}-d_{6}\right) \delta$ 1.182-1.869 (m, 10H, H cyclohexyl), 4.071 (s, 1H, H-1 cyclohexyl), 5.599 (s, 2H, $\mathrm{NH}_{2}$ ), 6.693 (d, 1H, NH), 7.638 (s, 1H, H-8).

\section{2-amino-6-phenylamino purine (3b)}

White powder; mp $290{ }^{\circ} \mathrm{C} .{ }^{1} \mathrm{H}$ NMR (DMSO- $\left.d_{6}\right) \delta$ $6.000\left(\mathrm{~s}, 2 \mathrm{H}, \mathrm{NH}_{2}\right), 6.966\left(\mathrm{t}, 1 \mathrm{H}, J 7.6 \mathrm{~Hz}, \mathrm{H}_{\mathrm{Ar}}\right), 7.275(\mathrm{t}$, $\left.2 \mathrm{H}, J 7.6 \mathrm{~Hz}, \mathrm{H}_{\mathrm{Ar}}\right), 7.816(\mathrm{~s}, 1 \mathrm{H}, \mathrm{H}-8), 8.015$ (d, 2H, J 7.6 $\mathrm{Hz}, \mathrm{H}_{\mathrm{Ar}}$ ), 9.254 (s, 1H, NH), 12.274 (bs, 1H, H-9). ${ }^{13} \mathrm{C}$ NMR (DMSO- $\left.d_{6}\right) \delta 113.55$ (5-C), 120.43, 122.07, 128.73, $136.89\left(\mathrm{C}_{\mathrm{Ar}}\right), 140.92$ (8-C), 152.38 (6-C), 153.32 (4-C), $160.18(2-\mathrm{C})$.

\section{2-amino-6-(p-tolylamino) purine $(3 \mathrm{c})$}

Lustrous flakes; mp 242-245 ${ }^{\circ} \mathrm{C} .{ }^{1} \mathrm{H}$ NMR (DMSOd) $\delta 2.262\left(\mathrm{~s}, 3 \mathrm{H}, \mathrm{CH}_{3}\right), 5.942\left(\mathrm{~s}, 2 \mathrm{H}, \mathrm{NH}_{2}\right), 7.075(\mathrm{~d}$, $\left.2 \mathrm{H}, J 8.4 \mathrm{~Hz}, \mathrm{H}_{\mathrm{Ar}}\right), 7.869$ (d, 2H, J $\left.8.4 \mathrm{~Hz}, \mathrm{H}_{\mathrm{Ar}}\right), 8.085$ (s, $1 \mathrm{H}, \mathrm{H}-8), 9.132$ (s, $1 \mathrm{H}, \mathrm{NH}) .{ }^{13} \mathrm{C}$ NMR (DMSO- $\left.d_{6}\right) \delta 20.87$ $\left(\mathrm{CH}_{3}\right), 119.75$ (5-C), 120.57, 123.67, 129.13, 130.85,
136.50, $138.37\left(\mathrm{C}_{\mathrm{Ar}}\right), 141.75$ (8-C), 149.48 (6-C), 155.41 (4-C), 160.29 (2-C).

\section{2-amino-6-(p-methoxyphenylamino) purine (3d)}

White powder; mp $290{ }^{\circ} \mathrm{C}$ (dec.). ${ }^{1} \mathrm{H}$ NMR (DMSO$\left.d_{6}\right) \delta 3.760\left(\mathrm{~s}, 3 \mathrm{H}, \mathrm{OCH}_{3}\right), 6.616\left(\mathrm{~s}, 2 \mathrm{H}, \mathrm{NH}_{2}\right), 6.922(\mathrm{~d}$, $\left.2 \mathrm{H}, J 8.8 \mathrm{~Hz}, \mathrm{H}_{\mathrm{Ar}}\right), 7.846$ (d, 2H, J $8.8 \mathrm{~Hz}, \mathrm{H}_{\mathrm{Ar}}$ ), 8.043 (s, $1 \mathrm{H}, \mathrm{H}-8), 9.603$ (s, $1 \mathrm{H}, \mathrm{NH}) .{ }^{13} \mathrm{C} \mathrm{NMR}\left(\mathrm{DMSO}-d_{6}\right) \delta 55.61$ $\left(\mathrm{OCH}_{3}\right), 119.66(5-\mathrm{C}), 114.04,122.91,133.27\left(\mathrm{C}_{\mathrm{Ar}}\right)$, 139.96 (8-C), 150.64 (6-C), 152.37 (4-C), 155.38 (2-C).

\section{2-amino-6-(p-ethoxyphenylamino) purine (3e)}

White powder; mp $133-135{ }^{\circ} \mathrm{C} .{ }^{1} \mathrm{H}$ NMR (DMSO- $d_{6}$ ) $\delta 1.317$ (t, 3H, J $6.8 \mathrm{~Hz}, \mathrm{CH}_{2} \mathrm{CH}_{3}$ ), 3.990 (q, 2H, J 6.8 $\left.\mathrm{Hz}, \mathrm{OCH}_{2} \mathrm{CH}_{3}\right), 6.022\left(\mathrm{~s}, 2 \mathrm{H}, \mathrm{NH}_{2}\right), 6.845$ (d, 2H, J 8.8 $\left.\mathrm{Hz}, \mathrm{H}_{\mathrm{Ar}}\right), 7.653$ (d, 2H, J $8.8 \mathrm{~Hz}, \mathrm{H}_{\mathrm{Ar}}$ ), 8.093 (s, 1H, H-8), 9.226 (s, 1H, NH), 12.848 (br, $1 \mathrm{H}, \mathrm{H}-9) .{ }^{13} \mathrm{C} \mathrm{NMR}$ $\left(\mathrm{DMSO}-d_{6}\right) \delta 15.19\left(\mathrm{CH}_{2} \mathrm{CH}_{3}\right), 63.53\left(\mathrm{CH}_{2} \mathrm{CH}_{3}\right), 119.16$ (5-C), 114.56, 122.24, 123.67, 133.75, $136.92\left(\mathrm{C}_{\mathrm{Ar}}\right)$, 141.77 (8-C), 149.48 (6-C), 152.77 (4-C), 154.21 (2-C).

\section{9- $\beta$-cyanoethyl-6-cyclohexylamino purine (4a)}

White needle crystal; mp 137-139 ${ }^{\circ} \mathrm{C} .{ }^{1} \mathrm{H}$ NMR (DMSO$\left.d_{6}\right) \delta$ 1.112-1.881 (m, 10H, H cyclohexyl), 3.168 (t, 2H, $J$ $6.4 \mathrm{~Hz}, \mathrm{CH}_{2} \mathrm{CH}_{2} \mathrm{CN}$ ), 4.101 (br, $1 \mathrm{H}, \mathrm{H}$ cyclohexyl), 4.447 (t, 2H, J $6.4 \mathrm{~Hz}, \mathrm{NCH}_{2} \mathrm{CH}_{2}$ ), 7.554 (d, 1H, J $8.0 \mathrm{~Hz}, \mathrm{NH}$ ), 8.187 (s, 1H, H-2), 8.219 (s, 1H, H-8). ${ }^{13} \mathrm{C}$ NMR (DMSO$\left.d_{6}\right) \delta 18.56\left(\mathrm{CH}_{2} \mathrm{CN}\right), 39.26\left(\mathrm{NCH}_{2} \mathrm{CH}_{2}\right), 24.80,25.56$, 26.25, 32.78, 33.35, 49.11 (C cyclohexy), $118.72(\mathrm{CN})$, 119.33 (5-C), 140.59 (8-C), 149.18 (6-C), 153.03 (4-C), 154.30 (2-C). MS (ESI) $m / z$ [ $\left[\mathrm{M}^{+} \mathrm{Na}^{+}-1\right.$ 307.8], 270.8, 254.8, 228.8, 203.7, 150.8. IR (KBr) $v_{\max } / \mathrm{cm}^{-1}: 3398,3385,3084$, 3036, 2933, 2854, 2250, 1607, 1586, 1475, 1366, 1299, 772. Anal. Calc. for $\mathrm{C}_{14} \mathrm{H}_{18} \mathrm{~N}_{6}$ : C, 62.22; H, 6.67; N, 31.11. Found: C, 61.97; H, 6.73; N, 31.34\%.

\section{9-3-cyanoethyl-6-phenylamino purine (4b)}

Lustrous flakes; mp 168-170 ${ }^{\circ} \mathrm{C}$. ${ }^{1} \mathrm{H}$ NMR (DMSO$\left.d_{6}\right) \delta 3.229\left(\mathrm{t}, 2 \mathrm{H}, J 6.4 \mathrm{~Hz}, \mathrm{CH}_{2} \mathrm{CH}_{2} \mathrm{CN}\right), 4.538(\mathrm{t}, 2 \mathrm{H}, J$ $\left.6.4 \mathrm{~Hz}, \mathrm{NCH}_{2} \mathrm{CH}_{2}\right), 7.049$ (t, 1H, J $6.4 \mathrm{~Hz}, \mathrm{H}_{\mathrm{Ar}}$ ), 7.340 (t, $\left.2 \mathrm{H}, J 6.0 \mathrm{~Hz}, \mathrm{H}_{\mathrm{Ar}}\right), 7.972$ (d, 2H, J $8.8 \mathrm{~Hz}, \mathrm{H}_{\mathrm{Ar}}$ ), 8.390 (s, $1 \mathrm{H}, \mathrm{H}-2), 8.445$ (s, 1H, H-8), 9.917 (s, 1H, NH). ${ }^{13} \mathrm{C} \mathrm{NMR}$ $\left(\mathrm{DMSO}-d_{6}\right) \delta 18.63\left(\mathrm{CH}_{2} \mathrm{CN}\right), 39.46\left(\mathrm{NCH}_{2} \mathrm{CH}_{2}\right), 118.71$ (CN), 120.20 (C-5), 121.33, 123.10, 128.82, $140.06\left(\mathrm{C}_{\mathrm{Ar}}\right)$, 141.94 (C-8), 149.75 (C-6), 150.04 (C-4), 152.53 (C-2). MS (ESI) $m / z$ [ $\left[\mathrm{M}^{+} \mathrm{Na}^{+}-1286.7\right], 233.8 . \mathrm{IR}(\mathrm{KBr}) \mathrm{v}_{\max } / \mathrm{cm}^{-}$ ${ }^{1}$ : 3352, 3087, 3050, 2967, 2261, 1622, 1580, 1476, 1300, 
1239, 1148, 1018, 753. Anal. Calc. for $\mathrm{C}_{14} \mathrm{H}_{12} \mathrm{~N}_{6}: \mathrm{C}, 63.64$; H, 4.54; N, 31.82. Found: C, 63.51; H, 4.59; N, 31.93\%.

9- $\beta$-cyanoethyl-6-(p-tolylamino) purine (4c)

Broken-white powder; mp 173-174 ${ }^{\circ} \mathrm{C} .{ }^{1} \mathrm{H}$ NMR $\left(\right.$ DMSO- $\left.d_{6}\right) \delta 2.284\left(\mathrm{~s}, 3 \mathrm{H}, \mathrm{CH}_{3}\right), 3.218(\mathrm{t}, 2 \mathrm{H}, J 6.4 \mathrm{~Hz}$, $\mathrm{CH}_{2} \mathrm{CH}_{2} \mathrm{CN}$ ), 4.521 (t, $\left.2 \mathrm{H}, J 6.4 \mathrm{~Hz}, \mathrm{NCH}_{2} \mathrm{CH}_{2}\right), 7.141$ $\left(\mathrm{d}, 2 \mathrm{H}, J 8.4 \mathrm{~Hz}, \mathrm{H}_{\mathrm{Ar}}\right), 7.816\left(\mathrm{~d}, 2 \mathrm{H}, J 8.4 \mathrm{~Hz}, \mathrm{H}_{\mathrm{Ar}}\right), 8.364$ (s, $1 \mathrm{H}, \mathrm{H}-2), 8.403(\mathrm{~s}, 1 \mathrm{H}, \mathrm{H}-8), 9.811(\mathrm{~s}, 1 \mathrm{H}, \mathrm{NH}) .{ }^{13} \mathrm{C}$ NMR (DMSO- $\left.d_{6}\right) \delta 18.60\left(\mathrm{CH}_{2} \mathrm{CN}\right), 20.91\left(\mathrm{CH}_{3}\right), 39.42$ $\left(\mathrm{NCH}_{2} \mathrm{CH}_{2}\right), 118.71(\mathrm{CN}), 120.07$ (C-5), 121.42, 129.23, 132.06, $137.46\left(\mathrm{C}_{\mathrm{Ar}}\right), 141.78$ (C-8), 149.93 (C-6), 151.23 (C-4), 152.56 (C-2). MS (ESI) $\mathrm{m} / z\left[\mathrm{M}^{+} \mathrm{Na}^{+}-1\right.$ 300.7], 145.9. IR (KBr) $v_{\text {max }} / \mathrm{cm}^{-1}: 3386,3089,2919,2860,2249$, 1616, 1585, 1476, 1367, 821. Anal. Calc. for $\mathrm{C}_{15} \mathrm{H}_{14} \mathrm{~N}_{6}$ : C, 64.75; H, 5.04; N, 30.22. Found: C, 64.49; H, 5.21; $\mathrm{N}, 30.35 \%$.

\section{9-3-cyanoethyl-6-(p-methoxyphenylamino) purine $(\mathbf{4 d})$}

Colorless column crystal; mp 80-82 ${ }^{\circ} \mathrm{C} .{ }^{1} \mathrm{H}$ NMR $\left(\mathrm{DMSO}-d_{6}\right) \delta 3.216$ (t, $2 \mathrm{H}, J 6.4 \mathrm{~Hz}, \mathrm{CH}_{2} \mathrm{CH}_{2} \mathrm{CN}$ ), 3.750 (s, $\left.3 \mathrm{H}, \mathrm{OCH}_{3}\right), 4.517$ (t, $\left.2 \mathrm{H}, J 6.4 \mathrm{~Hz}, \mathrm{NCH}_{2} \mathrm{CH}_{2}\right), 6.924$ $\left(\mathrm{d}, 2 \mathrm{H}, J 8.8 \mathrm{~Hz}, \mathrm{H}_{\mathrm{Ar}}\right), 7.794\left(\mathrm{~d}, 2 \mathrm{H}, J 9.2 \mathrm{~Hz}, \mathrm{H}_{\mathrm{Ar}}\right), 8.344$ (s, 1H, H-2), 8.370 (s, 1H, H-8), 9.765 (s, $1 \mathrm{H}, \mathrm{NH}) .{ }^{13} \mathrm{C}$ NMR (DMSO- $\left.d_{6}\right) \delta 18.62\left(\mathrm{CH}_{2} \mathrm{CN}\right), 39.41\left(\mathrm{NCH}_{2} \mathrm{CH}_{2}\right)$, $55.64\left(\mathrm{OCH}_{3}\right), 118.72(\mathrm{CN}), 119.91(\mathrm{C}-5), 114.06,123.23$, 132.95, $152.63\left(\mathrm{C}_{\mathrm{Ar}}\right), 141.61(\mathrm{C}-8), 149.82(\mathrm{C}-6), 152.67$ (C-4), 155.61 (C-2). MS (ESI) $m / z$ [ $\left.\mathrm{M}^{+} \mathrm{Na}^{+}-1316.7\right], 263.7$. IR (KBr) $v_{\max } / \mathrm{cm}^{-1}: 3466,3301,3204,3101,2978,2881$, 2253, 1620, 1587, 1512, 1471, 1294, 1035, 797. Anal. Calc. for $\mathrm{C}_{15} \mathrm{H}_{14} \mathrm{~N}_{6} \mathrm{O}: \mathrm{C}, 61.22 ; \mathrm{H}, 4.76 ; \mathrm{N}, 28.57$. Found: C, 61.09; H, 4.84; N, 28.64\%.

\section{9-ß-cyanoethyl-6-(p-ethoxyphenylamino) purine (4e)}

Gray flakes; mp $146-148{ }^{\circ} \mathrm{C} .{ }^{1} \mathrm{H}$ NMR (DMSO- $\left.d_{6}\right) \delta$ $1.328\left(\mathrm{t}, 3 \mathrm{H}, J 7.2 \mathrm{~Hz}, \mathrm{CH}_{2} \mathrm{CH}_{3}\right), 3.215(\mathrm{t}, 2 \mathrm{H}, J 6.4 \mathrm{~Hz}$, $\mathrm{CH}_{2} \mathrm{CH}_{2} \mathrm{CN}$ ), 4.009 (q, $2 \mathrm{H}, J 7.2 \mathrm{~Hz}, \mathrm{OCH}_{2} \mathrm{CH}_{3}$ ), 4.513 $\left(\mathrm{t}, 2 \mathrm{H}, J 6.4 \mathrm{~Hz}, \mathrm{NCH}_{2} \mathrm{CH}_{2}\right), 6.907(\mathrm{~d}, 2 \mathrm{H}, J 9.2 \mathrm{~Hz}$, $\left.\mathrm{H}_{\mathrm{Ar}}\right), 7.783\left(\mathrm{~d}, 2 \mathrm{H}, J 8.8 \mathrm{~Hz}, \mathrm{H}_{\mathrm{Ar}}\right), 8.343(\mathrm{~s}, 1 \mathrm{H}, \mathrm{H}-2)$, 8.365 (s, $1 \mathrm{H}, \mathrm{H}-8), 9.758$ (s, $1 \mathrm{H}, \mathrm{NH}) .{ }^{13} \mathrm{C}$ NMR (DMSO$\left.d_{6}\right) \delta 15.16\left(\mathrm{OCH}_{2} \mathrm{CH}_{3}\right), 18.60\left(\mathrm{CH}_{2} \mathrm{CN}\right), 39.42$ $\left(\mathrm{NCH}_{2} \mathrm{CH}_{2}\right), 118.72(\mathrm{CN}), 119.90(\mathrm{C}-5), 114.60,123.15$, $132.88,152.62\left(\mathrm{C}_{\mathrm{Ar}}\right), 141.61(\mathrm{C}-8), 149.81(\mathrm{C}-6), 154.84$ (C-4), 158.64 (C-2). MS (ESI) $m / z\left[\mathrm{M}^{+} \mathrm{Na}^{+}-1330.8\right]$, 308.8. IR (KBr) $v_{\max } / \mathrm{cm}^{-1}: 3309,3231,3107,2979,1930$, 2881, 2252, 1618, 1587, 1512, 1479, 1299, 1048, 840. Anal. Calc. for $\mathrm{C}_{16} \mathrm{H}_{16} \mathrm{~N}_{6} \mathrm{O}: \mathrm{C}, 62.34 ; \mathrm{H}, 5.19 ; \mathrm{N}, 27.27$. Found: C, 62.11; H, 5.31; N, 27.46\%. 2-chloro-9-3-cyanoethyl-6-cyclohexylamino purine (5a)

White powder; mp 179-181 ${ }^{\circ} \mathrm{C} .{ }^{1} \mathrm{H}$ NMR (DMSO- $\left.d_{6}\right) \delta$ 1.107-1.923 (m, 10H, H cyclohexyl), 3.135 (t, 2H, J 6.4Hz, $\mathrm{CH}_{2} \mathrm{CH}_{2} \mathrm{CN}$ ), 4.005 (br, 1H, H cyclohexyl), 4.411 (t, $2 \mathrm{H}, J$ $6.4 \mathrm{~Hz}, \mathrm{NCH}_{2} \mathrm{CH}_{2}$ ), 8.163 (d, 1H, J $8.0 \mathrm{~Hz}, \mathrm{NH}$ ), 8.199 (s, $1 \mathrm{H}, \mathrm{H}-8) .{ }^{13} \mathrm{C}$ NMR (DMSO- $\left.d_{6}\right) \delta 18.62\left(\mathrm{CH}_{2} \mathrm{CN}\right), 25.13$, 25.36, 25.56, 32.47, 33.48, 49.45 (C cyclohexyl), 38.76 $\left(\mathrm{NCH}_{2} \mathrm{CH}_{2}\right), 118.38(\mathrm{C}-5), 118.65(\mathrm{CN}), 141.15(\mathrm{C}-8)$, 150.13 (C-6), 153.79 (C-4), 154.66 (C-2). MS (ESI) $\mathrm{m} / \mathrm{z}$ $\left[\mathrm{M}^{+} \mathrm{Na}^{+}-1,286.7\right], 233.8$. Anal. Calc. for $\mathrm{C}_{14} \mathrm{H}_{17} \mathrm{ClN}_{6}: \mathrm{C}$, 55.17; H, 5.62; N, 27.57. Found: C, 54.97; H, 5.69; N, $27.73 \%$.

\section{2-chloro-9- $\beta$-cyanoethyl-6-phenylamino purine (5b)}

White powder; mp 266-268 ${ }^{\circ} \mathrm{C} .{ }^{1} \mathrm{H}$ NMR (DMSO- $d_{6}$ ) $\delta 3.187$ (t, 2H, J $6.4 \mathrm{~Hz}, \mathrm{CH}_{2} \mathrm{CH}_{2} \mathrm{CN}$ ), 4.492 (t, 2H, J 6.4 $\left.\mathrm{Hz}, \mathrm{NCH}_{2} \mathrm{CH}_{2}\right), 7.113\left(\mathrm{t}, 1 \mathrm{H}, J 7.2 \mathrm{~Hz}, \mathrm{H}_{\mathrm{Ar}}\right), 7.374(\mathrm{t}, 2 \mathrm{H}$, $\left.J 7.6 \mathrm{~Hz}, \mathrm{H}_{\mathrm{Ar}}\right), 7.845\left(\mathrm{~d}, 2 \mathrm{H}, J 8.0 \mathrm{~Hz}, \mathrm{H}_{\mathrm{Ar}}\right), 8.388(\mathrm{~s}, 1 \mathrm{H}$, $\mathrm{H}-8), 10.348$ (s, 1H, NH). ${ }^{13} \mathrm{C}$ NMR (DMSO- $d_{6}$ ) $\delta 18.64$ $\left(\mathrm{CH}_{2} \mathrm{CN}\right), 39.94\left(\mathrm{NCH}_{2} \mathrm{CH}_{2}\right), 118.65(\mathrm{CN}), 119.20(\mathrm{C}-5)$, 121.81, 124.03, 128.97, $139.14\left(\mathrm{C}_{\mathrm{Ar}}\right), 142.49(\mathrm{C}-8), 149.32$ (C-6), 151.15 (C-4), 152.91 (C-2). MS (ESI) $m / z,\left[\mathrm{M}^{+} \mathrm{Na}^{+}-\right.$ 1, 320.7], 298.8, 256.9, 101.9, 88.0. IR (KBr) $v_{\max } / \mathrm{cm}^{-1}$ : 3346, 3087, 3062, 2973, 2935, 2258, 1622, 1578, 1500, 1452, 1318, 1284, 756. Anal. Calc. for $\mathrm{C}_{14} \mathrm{H}_{11} \mathrm{ClN}_{6}: \mathrm{C}$, 56.28; H, 3.69; N, 28.14. Found: C, 55.97; H, 3.81; N, $28.36 \%$.

2-chloro-9- $\beta$-cyanoethyl-6-(p-tolylamino) purine (5c)

Lustrous needle crystal; mp 254-256 ${ }^{\circ} \mathrm{C} .{ }^{1} \mathrm{H}$ NMR $\left(\right.$ DMSO- $\left.d_{6}\right) \delta 2.297$ (s, 3H, $\mathrm{CH}_{3}$ ), 3.180 (t, $2 \mathrm{H}, J 6.4 \mathrm{~Hz}$, $\mathrm{CH}_{2} \mathrm{CH}_{2} \mathrm{CN}$ ), 4.480 (t, $2 \mathrm{H}, J 6.4 \mathrm{~Hz}, \mathrm{NCH}_{2} \mathrm{CH}_{2}$ ), 7.174 (d, $\left.2 \mathrm{H}, J 8.0 \mathrm{~Hz}, \mathrm{H}_{\mathrm{Ar}}\right), 7.690\left(\mathrm{~d}, 2 \mathrm{H}, J 8.4 \mathrm{~Hz}, \mathrm{H}_{\mathrm{Ar}}\right), 8.363$ (s, $1 \mathrm{H}, \mathrm{H}-8), 10.256$ (s, $1 \mathrm{H}, \mathrm{NH}$ ). ${ }^{13} \mathrm{C}$ NMR (DMSO- $d_{6}$ ) $\delta 18.63$ $\left(\mathrm{CH}_{2} \mathrm{CN}\right), 20.94\left(\mathrm{CH}_{3}\right), 39.99\left(\mathrm{NCH}_{2} \mathrm{CH}_{2}\right), 118.65(\mathrm{CN})$, 119.08 (C-5), 121.99, 129.39, 133.18, $136.51\left(\mathrm{C}_{\mathrm{Ar}}\right), 142.32$ (C-8), 149.54 (C-6), 151.04 (C-4), 152.98 (C-2). MS (ESI) $m / z$ [ $\left.\mathrm{M}^{+} \mathrm{Na}^{+}-1,334.7\right], 271.9,227.9,145.8,96.9$. IR (KBr) $v_{\max } / \mathrm{cm}^{-1}: 3339,3055,2930,2264,1623,1579,1514,1456$, 1252, 818. Anal. Calc. for $\mathrm{C}_{15} \mathrm{H}_{13} \mathrm{ClN}_{6}$ : C, 57.60; H, 4.16; $\mathrm{N}, 26.88$. Found: C, 57.51; H, 4.22; N, 26.93\%.

2-chloro-9- $\beta$-cyanoethyl-6-(p-methoxyphenylamino) purine (5d)

Lustrous flakes; mp 239-240 ${ }^{\circ} \mathrm{C} .{ }^{1} \mathrm{H}$ NMR (DMSO$\left.d_{6}\right) \delta 3.178\left(\mathrm{t}, 2 \mathrm{H}, J 6.4 \mathrm{~Hz}, \mathrm{CH}_{2} \mathrm{CH}_{2} \mathrm{CN}\right), 3.764(\mathrm{~s}, 3 \mathrm{H}$, $\left.\mathrm{OCH}_{3}\right), 4.476\left(\mathrm{t}, 2 \mathrm{H}, \mathrm{J} 6.4 \mathrm{~Hz}, \mathrm{NCH}_{2} \mathrm{CH}_{2}\right), 6.954(\mathrm{~d}, 2 \mathrm{H}$, 
$\left.J 8.8 \mathrm{~Hz}, \mathrm{H}_{\mathrm{Ar}}\right), 7.691\left(\mathrm{~d}, 2 \mathrm{H}, J 8.8 \mathrm{~Hz}, \mathrm{H}_{\mathrm{Ar}}\right), 8.343(\mathrm{~s}, 1 \mathrm{H}$, $\mathrm{H}-8$ ), 10.205 (s, $1 \mathrm{H}, \mathrm{NH}$ ). ${ }^{13} \mathrm{C}$ NMR (DMSO- $\left.d_{6}\right) \delta 18.65$ $\left(\mathrm{CH}_{2} \mathrm{CN}\right), 39.49\left(\mathrm{NCH}_{2} \mathrm{CH}_{2}\right), 55.68\left(\mathrm{OCH}_{3}\right), 118.65(\mathrm{CN})$, 118.94 (C-5), 114.20, 123.71, 131.96, $150.91\left(\mathrm{C}_{\mathrm{Ar}}\right), 142.15$ (C-8), 153.06 (C-6), 153.12 (C-4), 156.23 (C-2). MS (ESI) $\mathrm{m} / z$ [ $\left.\mathrm{M}^{+} \mathrm{Na}^{+}-1,350.7\right], 328.8,292.8,239.8,224.7,196.7$. IR $(\mathrm{KBr}) v_{\max } / \mathrm{cm}^{-1}: 3337,3063,2962,2935,2835,2258$, 1624, 1589, 1512, 1478, 1309, 1248, 1048, 827. Anal. Calc. for $\mathrm{C}_{15} \mathrm{H}_{13} \mathrm{ClN}_{6} \mathrm{O}: \mathrm{C}, 54.79 ; \mathrm{H}, 3.96 ; \mathrm{N}, 25.57$. Found: C, 54.66; H, 4.03; N, 25.64\%.

2-amino-9- $\beta$-cyanoethyl-6-cyclohexylamino purine (6a)

White powder; mp $135-136{ }^{\circ} \mathrm{C} .{ }^{1} \mathrm{H}$ NMR (DMSO- $d_{6}$ ) $\delta$ 1.085-1.861 (m, 10H, H cyclohexyl), 3.088 (t, 2H, $J$ $6.4 \mathrm{~Hz}, \mathrm{CH}_{2} \mathrm{CH}_{2} \mathrm{CN}$ ), 4.060 (br, 1H, H cyclohexyl), 4.243 (t, $\left.2 \mathrm{H}, J 6.4 \mathrm{~Hz}, \mathrm{NCH}_{2} \mathrm{CH}_{2}\right), 5.846\left(\mathrm{~s}, 2 \mathrm{H}, \mathrm{NH}_{2}\right), 6.914$ (s, $1 \mathrm{H}, \mathrm{NH}$ ), 7.742 (s, 1H, H-8). ${ }^{13} \mathrm{C}$ NMR (DMSO- $d_{6}$ ) $\delta 18.34$ $\left(\mathrm{CH}_{2} \mathrm{CN}\right), 25.51,25.69,33.13,48.54$ (C cyclohexy), 38.76 $\left(\mathrm{NCH}_{2} \mathrm{CH}_{2}\right), 113.48(\mathrm{C}-5), 118.85(\mathrm{CN}), 137.04(\mathrm{C}-8)$, 152.31 (C-6), 154.63 (C-4), 160.76 (C-2). MS (ESI) $\mathrm{m} / \mathrm{z}$ $\left[\mathrm{M}^{+} \mathrm{Na}^{+}-1,307.8\right], 285.9,254.8,203.7,150.8$. IR (KBr) $\mathrm{V}_{\max } / \mathrm{cm}^{-1}: 3331,3218,3103,2929,2854,2246,1637$, 1602, 1486, 1399, 791. Anal. Calc. for $\mathrm{C}_{14} \mathrm{H}_{19} \mathrm{~N}_{7}$ : C, 58.95; H, 6.67; N, 34.39. Found: C, 58.82; H, 6.79; N, 34.45\%.

\section{2-amino-9-3-cyanoethyl-6-phenylamino purine (6b)}

Colorless flake crystal; mp 246-247 ${ }^{\circ} \mathrm{C} .{ }^{1} \mathrm{H}$ NMR (DMSO- $d_{6}$ ) $\delta 3.141$ (t, $2 \mathrm{H}, J 6.4 \mathrm{~Hz}, \mathrm{CH}_{2} \mathrm{CH}_{2} \mathrm{CN}$ ), 4.313 (t, $2 \mathrm{H}, J 6.4 \mathrm{~Hz}, \mathrm{NCH}_{2} \mathrm{CH}_{2}$ ), 6.204 (s, 2H, $\mathrm{NH}_{2}$ ), 6.981 (t, $1 \mathrm{H}, J$ $\left.7.2 \mathrm{~Hz}, \mathrm{H}_{\mathrm{Ar}}\right), 7.280\left(\mathrm{t}, 2 \mathrm{H}, J 8.0 \mathrm{~Hz}, \mathrm{H}_{\mathrm{Ar}}\right), 7.910$ (s, $\left.1 \mathrm{H}, \mathrm{H}-8\right)$, $8.017\left(\mathrm{~d}, 2 \mathrm{H}, J 8.0 \mathrm{~Hz}, \mathrm{H}_{\mathrm{Ar}}\right), 9.380(\mathrm{~s}, 1 \mathrm{H}, \mathrm{NH}) .{ }^{13} \mathrm{C} \mathrm{NMR}$ $\left(\mathrm{DMSO}-d_{6}\right) \delta 18.36\left(\mathrm{CH}_{2} \mathrm{CN}\right), 38.87\left(\mathrm{NCH}_{2} \mathrm{CH}_{2}\right), 118.83$ $(\mathrm{CN}), 120.65$ (C-5), 114.17, 122.25, 128.71, $138.13\left(\mathrm{C}_{\mathrm{Ar}}\right)$, 140.77 (C-8), 152.18 (C-6), 152.82 (C-4), 160.44 (C-2). MS (ESI) $m / z\left[\mathrm{M}^{+} \mathrm{Na}^{+}-1,301.6\right], 248.7 . \mathrm{IR}(\mathrm{KBr}) v_{\max } / \mathrm{cm}^{-1}: 3445$, 3391, 3329, 3202, 3099, 2984, 2935, 2252, 1645, 1617, 1579, 1498, 1439, 787. Anal. Calc. for $\mathrm{C}_{14} \mathrm{H}_{13} \mathrm{~N}_{7}$ : C, 60.22; H, 4.66; N, 35.13. Found: C, 60.13; H, 4.73; N, 35.19\%.

\section{2-amino-9- $\beta$-cyanoethyl-6-(p-tolylamino) purine (6c)}

White needle crystal; mp 208-209 ${ }^{\circ} \mathrm{C} .{ }^{1} \mathrm{H}$ NMR (DMSO$\left.d_{6}\right) \delta 2.269$ (s, 3H, $\left.\mathrm{CH}_{3}\right), 3.134$ (t, $2 \mathrm{H}, \mathrm{J} 6.4 \mathrm{~Hz}, \mathrm{CH}_{2} \mathrm{CH}_{2} \mathrm{CN}$ ), 4.305 (t, $\left.2 \mathrm{H}, J 6.4 \mathrm{~Hz}, \mathrm{NCH}_{2} \mathrm{CH}_{2}\right), 6.153$ (s, $\left.2 \mathrm{H}, \mathrm{NH}_{2}\right), 7.083$ (d, $2 \mathrm{H}, J 8.4 \mathrm{~Hz}, \mathrm{H}_{\mathrm{Ar}}$ ), 7.864 (d, 2H, J $8.4 \mathrm{~Hz}, \mathrm{H}_{\mathrm{Ar}}$ ), 7.889 (s, $1 \mathrm{H}, \mathrm{H}-8), 9.271$ (s, $1 \mathrm{H}, \mathrm{NH}) .{ }^{13} \mathrm{C}$ NMR (DMSO- $d_{6}$ ) $\delta 18.36$ $\left(\mathrm{CH}_{2} \mathrm{CN}\right), 20.89\left(\mathrm{CH}_{3}\right), 38.86\left(\mathrm{NCH}_{2} \mathrm{CH}_{2}\right), 118.83(\mathrm{CN})$, 120.82 (C-5), 114.10, 129.14, 131.13, $137.98\left(\mathrm{C}_{\mathrm{Ar}}\right), 138.17$ (C-8), 152.06 (C-6), 152.87 (C-4), 160.46 (C-2). MS (ESI) $m / z\left[\mathrm{M}^{+} \mathrm{Na}^{+}-1,315.8\right], 293.8,245.9,228.8,198.7,156.8$, 144.7, 117.9, 82.0, 65.1. IR (KBr) $v_{\max } / \mathrm{cm}^{-1}: 3457,3337$, 3104, 2944, 2855, 2252, 1627, 1597, 1513, 1482, 1417, 825, 788. Anal. Calc. for $\mathrm{C}_{15} \mathrm{H}_{15} \mathrm{~N}_{7}$ : C, 61.43; H, 5.12; N, 33.45 . Found: C, 61.36; H, 5.17; N, 33.48\%.

2-amino-9- $\beta$-cyanoethyl-6-(p-methoxyphenylamino) purine $(\boldsymbol{6 d})$

Colorless needle crystal; mp 174-176 ${ }^{\circ} \mathrm{C} .{ }^{1} \mathrm{H}$ NMR (DMSO- $d_{6}$ ) $\delta 3.133$ (t, $2 \mathrm{H}, J 6.4 \mathrm{~Hz}, \mathrm{CH}_{2} \mathrm{CH}_{2} \mathrm{CN}$ ), 3.738 (s, $3 \mathrm{H}, \mathrm{OCH}_{3}$ ), 4.303 (t, $2 \mathrm{H}, J 6.4 \mathrm{~Hz}, \mathrm{NCH}_{2} \mathrm{CH}_{2}$ ), 6.116 $\left(\mathrm{s}, 2 \mathrm{H}, \mathrm{NH}_{2}\right), 6.863\left(\mathrm{~d}, 2 \mathrm{H}, J 8.8 \mathrm{~Hz}, \mathrm{H}_{\mathrm{Ar}}\right), 7.856(\mathrm{~d}, 2 \mathrm{H}, J$ $8.8 \mathrm{~Hz}, \mathrm{H}_{\mathrm{Ar}}$ ), 7.877 (s, 1H, H-8), 9.249 (s, $\left.1 \mathrm{H}, \mathrm{NH}\right) .{ }^{13} \mathrm{C}$ NMR (DMSO- $\left.d_{6}\right) \delta 18.37\left(\mathrm{CH}_{2} \mathrm{CN}\right), 38.86\left(\mathrm{NCH}_{2} \mathrm{CH}_{2}\right)$, $55.61\left(\mathrm{OCH}_{3}\right), 118.84(\mathrm{CN}), 120.15(\mathrm{C}-5), 113.95,122.46$, 133.82, 137.86, $152.92\left(\mathrm{C}_{\mathrm{Ar}}\right), 141.59(\mathrm{C}-8), 151.96(\mathrm{C}-$ 6), 154.98 (C-4), 160.50 (C-2). MS (ESI) $\mathrm{m} / z\left[\mathrm{M}^{+} \mathrm{Na}^{+}-1\right.$, 331.7], 309.8, 292.8, 267.8, 252.7, 224.7, 214.7, 199.7, 160.8 , 92.4. IR (KBr) $v_{\max } / \mathrm{cm}^{-1}: 3483,3327,3203,3008$, 2946, 2848, 2250, 1596, 1514, 1486, 1243, 1031, 786. Anal. Calc. for $\mathrm{C}_{15} \mathrm{H}_{15} \mathrm{~N}_{7} \mathrm{O}: \mathrm{C}, 58.25 ; \mathrm{H}, 4.85 ; \mathrm{N}, 31.72$. Found: C, 58.16; H, 4.78; N, 31.91\%.

2-amino-9- $\beta$-cyanoethyl-6-(p-ethoxyphenylamino) purine (6e)

White needle crystal; mp $177-178{ }^{\circ} \mathrm{C} .{ }^{1} \mathrm{H}$ NMR (DMSO$\left.d_{6}\right) \delta 1.324\left(\mathrm{t}, 3 \mathrm{H}, J 6.8 \mathrm{~Hz}, \mathrm{OCH}_{2} \mathrm{CH}_{3}\right), 3.131$ (t, $2 \mathrm{H}, J 6.4 \mathrm{~Hz}$, $\mathrm{CH}_{2} \mathrm{CH}_{2} \mathrm{CN}$ ), 3.998 (q, $\left.2 \mathrm{H}, J 6.8 \mathrm{~Hz}, \mathrm{OCH}_{2} \mathrm{CH}_{3}\right), 4.301$ (t, $\left.2 \mathrm{H}, J 6.4 \mathrm{~Hz}, \mathrm{NCH}_{2} \mathrm{CH}_{2}\right), 6.114\left(\mathrm{~s}, 2 \mathrm{H}, \mathrm{NH}_{2}\right), 6.846(\mathrm{~d}, 2 \mathrm{H}$, $\left.J 8.8 \mathrm{~Hz}, \mathrm{H}_{\mathrm{Ar}}\right), 7.851\left(\mathrm{~d}, 2 \mathrm{H}, J 9.2 \mathrm{~Hz}, \mathrm{H}_{\mathrm{Ar}}\right), 7.876(\mathrm{~s}, 1 \mathrm{H}, \mathrm{H}-$ 8), $9.243(\mathrm{~s}, 1 \mathrm{H}, \mathrm{NH}) .{ }^{13} \mathrm{C}$ NMR (DMSO- $\left.d_{6}\right) \delta 15.20$ $\left(\mathrm{OCH}_{2} \mathrm{CH}_{3}\right), 18.60\left(\mathrm{CH}_{2} \mathrm{CN}\right), 38.86\left(\mathrm{NCH}_{2} \mathrm{CH}_{2}\right), 63.52$ $\left(\mathrm{OCH}_{2} \mathrm{CH}_{3}\right), 118.84(\mathrm{CN}), 120.15(\mathrm{C}-5), 113.97,114.52$, 122.41, 133.73, 137.85, $152.89\left(\mathrm{C}_{\mathrm{Ar}}\right), 141.59$ (C-8), 151.93 (C-6), 154.23 (C-4), 160.47 (C-2). MS (ESI) $m / z\left[\mathrm{M}^{+} \mathrm{Na}^{+}-1\right.$, 323.8], 281.8, 253.7, 224.7, 200.7, 146.8, 119.8, 80.9. IR (KBr) $v_{\max } / \mathrm{cm}^{-1}: 3487,3322,3195,2975,2930,2884,2248$, 1599, 1512, 1456, 1418, 1235, 1053, 832, 786. Anal. Calc. for $\mathrm{C}_{16} \mathrm{H}_{17} \mathrm{~N}_{7} \mathrm{O}: \mathrm{C}, 59.44 ; \mathrm{H}, 5.26$; N, 30.34. Found: C, 59.35; $\mathrm{H}, 5.29$; N, 30.42\%.

6-cyclohexylamino-9-(2-D-ribofuranosyl) purine (7a)

White flake crystal; mp $187-188^{\circ} \mathrm{C}$ (lit.187- $188^{\circ} \mathrm{C}$ ). ${ }^{7 \mathrm{c}}$ ${ }^{1} \mathrm{H} \mathrm{NMR}$ (DMSO- $\left.d_{6}\right) \delta 1.119-1.873$ (m, 10H, H cyclohexyl), 3.529-3.586, 3.685 (m, 2H, H-5'), 3.971 (m, 1H, H-4'), 4.096 (s, 1H, H-1 cyclohexyl), 4.150 (m, 1H, H-3'), 4.612 (m, 1H, H-2'), 5.881 (d, 1H, J $6.4 \mathrm{~Hz}, \mathrm{H}-1$ '), 7.628 (d, 1H, J $8.0 \mathrm{~Hz}, \mathrm{NH}), 8.191$ (s, 1H, H-2), 8.339 (s, 1H, H-8). 
6-phenylamino-9-( $\beta$-D-ribofuranosyl) purine (7b)

White powder; mp $195-196{ }^{\circ} \mathrm{C}$ (lit. $\left.195-196{ }^{\circ} \mathrm{C}\right) .{ }^{27}{ }^{1} \mathrm{H}$ NMR (DMSO- $\left.d_{6}\right) \delta 3.606$ (m, 2H, H-5'), $3.990(\mathrm{~m}, 1 \mathrm{H}$, H-4'), 4.192 (m, 1H, H-3'), 4.651 (m, 1H, H-2'), 5.970 (d, $\left.1 \mathrm{H}, J 6.0 \mathrm{~Hz}, \mathrm{H}-1^{\prime}\right), 7.050\left(\mathrm{t}, 1 \mathrm{H}, J 4.0 \mathrm{~Hz}, \mathrm{H}_{\mathrm{Ar}}\right), 7.341$ (t, $\left.2 \mathrm{H}, J 4.0 \mathrm{~Hz}, \mathrm{H}_{\mathrm{Ar}}\right), 7.947$ (d, 2H, J 4.0Hz, $\left.\mathrm{H}_{\mathrm{Ar}}\right), 8.407$ (s, 1H, H-2), 8.551 (s, 1H, H-8), 9.942 (s, 1H, NH). ${ }^{13} \mathrm{C}$ NMR $\left(\mathrm{DMSO}-d_{6}\right) \delta 60.21$ (5'-C), 70.99 (3'-C), 74.05 (2'-C), 86.30 (4'-C), 88.31 (1'-C), 117.19 (5-C), 120.81, 121.36, $123.17,128.83,129.32,139.98\left(\mathrm{C}_{\mathrm{Ar}}\right), 141.13(8-\mathrm{C}), 149.79$ (6-C), 152.36 (4-C), 152.62 (2-C).

\section{9-( $\beta$-D-ribofuranosyl)-6-(p-tolylamino)purine (7c)}

Buff needle crystal; mp 214-215 ${ }^{\circ} \mathrm{C}$ (lit. $\left.214-216^{\circ} \mathrm{C}\right) .^{7 \mathrm{a}}$ ${ }^{1} \mathrm{H}$ NMR (DMSO- $d_{6}$ ) $\delta 2.286$ (s, 3H, $\mathrm{CH}_{3}$ ), 3.645 (dd, 2H, $J$ 12 Hz, H-5'), 3.990 (q, 1H, J 3.2 Hz, H-4'), 4.181 (d, 1H, J $\left.3.2 \mathrm{~Hz}, \mathrm{H}-3^{\prime}\right), 4.646$ (q, 1H, J 6.4 Hz, H-2'), 5.959 (d, 1H, J $\left.7.0 \mathrm{~Hz}, \mathrm{H}-1^{\prime}\right), 7.143$ (d, 2H, J $8.4 \mathrm{~Hz}, \mathrm{H}_{\mathrm{Ar}}$ ), 7.808 (d, 2H, J $8.4 \mathrm{~Hz}, \mathrm{H}_{\mathrm{Ar}}$ ), 8.378 (s, 1H, H-2), 8.525 (s, 1H, H-8), 9.845 (s, $1 \mathrm{H}, \mathrm{NH}) .{ }^{13} \mathrm{C}$ NMR (DMSO- $\left.d_{6}\right) \delta 20.92\left(\mathrm{CH}_{3}\right), 62.02(5-\mathrm{C})$, 71.00 (3'-C), 74.04 (2'-C), 86.30 (4'-C), 88.32 (1'-C), 117.40 (5-C), 120.71, 121.48, 129.24, 132.16, $137.38\left(\mathrm{C}_{\mathrm{Ar}}\right), 140.98$ (8-C), 149.67 (6-C), 152.40 (4-C), 152.68 (2-C)

6-(p-methoxyphenylamino)-9-( $\beta$-D-ribofuranosyl) purine (7d)

Broken-white needle crystal; mp 206-208 ${ }^{\circ} \mathrm{C}$ (lit. 207 $\left.208{ }^{\circ} \mathrm{C}\right) .{ }^{7 \mathrm{a}}{ }^{1} \mathrm{H}$ NMR (DMSO- $\left.d_{6}\right) \delta 3.650$ (m, 2H, H-5'), $3.753\left(\mathrm{~s}, 3 \mathrm{H}, \mathrm{OCH}_{3}\right), 3.992(\mathrm{dd}, 1 \mathrm{H}, J 3.2$ and $3.6 \mathrm{~Hz}, \mathrm{H}-$ 4'), 4.181 (dd, 1H, J 3.2 and $4.8 \mathrm{~Hz}, \mathrm{H}-3$ '), 4.646 (dd, 1H, $J 4.8$ and $\left.6.0 \mathrm{~Hz}, \mathrm{H}-2^{\prime}\right), 5.952$ (d, 1H, J $\left.6.0 \mathrm{~Hz}, \mathrm{H}-1^{\prime}\right)$, $6.925\left(\mathrm{~m}, 2 \mathrm{H}, \mathrm{H}_{\mathrm{Ar}}\right), 7.786\left(\mathrm{~m}, 2 \mathrm{H}, \mathrm{H}_{\mathrm{Ar}}\right), 8.340(\mathrm{~s}, 1 \mathrm{H}, \mathrm{H}-$ 2), $8.504(\mathrm{~s}, 1 \mathrm{H}, \mathrm{H}-8), 9.800(\mathrm{~s}, 1 \mathrm{H}, \mathrm{NH}) .{ }^{13} \mathrm{C} \mathrm{NMR}$ $\left(\mathrm{DMSO}-d_{6}\right) \delta 55.66\left(\mathrm{OCH}_{3}\right), 62.04$ (5'-C), 71.02 (3'-C), 74.03 (2'-C), 86.31 (4'-C), 88.34 (1'-C), 117.42 (5-C), $114.07,120.56,123.26,132.88,149.55\left(\mathrm{C}_{\mathrm{Ar}}\right), 140.86(8-$ C), 152.45 (6-C), 152.77 (4-C), 155.65 (2-C).

6-(p-ethoxyphenylamino)-9-(2-D-ribofuranosyl) purine (7e)

White powder; mp 191-192 ${ }^{\circ} \mathrm{C} .{ }^{1} \mathrm{H}$ NMR (DMSO- $d_{6}$ ) $\delta 1.330\left(\mathrm{t}, 3 \mathrm{H}, J 7.2 \mathrm{~Hz}, \mathrm{CH}_{2} \mathrm{CH}_{3}\right), 3.650$ (dd, $2 \mathrm{H}, J 12$ Hz, H-5'), 3.898 (m, 1H, H-4'), 4.012 (q, 2H, J 7.2 Hz, $\left.\mathrm{OCH}_{2} \mathrm{CH}_{3}\right), 4.183$ (m, 1H, H-3'), 4.648 (m, 1H, H-2'), $5.953(\mathrm{~d}, 1 \mathrm{H}, J 7.0 \mathrm{~Hz}, \mathrm{H}-1$ ') 6.908 (d, 2H, J $8.8 \mathrm{~Hz}$, $\left.\mathrm{H}_{\mathrm{Ar}}\right), 7.777\left(\mathrm{~d}, 2 \mathrm{H}, J 8.8 \mathrm{~Hz}, \mathrm{H}_{\mathrm{Ar}}\right), 8.341(\mathrm{~s}, 1 \mathrm{H}, \mathrm{H}-2)$, 8.504 (s, 1H, H-8), 9.792 (s, 1H, NH). ${ }^{13} \mathrm{C} \mathrm{NMR} \mathrm{(DMSO-}$ $\left.d_{6}\right) \delta 15.16\left(\mathrm{CH}_{2} \mathrm{CH}_{3}\right), 62.05\left(5^{\prime}-\mathrm{C}\right), 63.57\left(\mathrm{CH}_{2} \mathrm{CH}_{3}\right), 71.03$
(3'-C), 74.03 (2'-C), 86.32 (4'-C), 88.35 (1'-C), 117.41 (5C), 114.61, 115.77, 120.56, 123.21, 132.78, $149.53\left(\mathrm{C}_{\mathrm{Ar}}\right)$, 140.85 (8-C), 152.45 (6-C), 152.75 (4-C), 154.89 (2-C). MS (ESI) $m / z+\mathrm{Na}^{+}-1409.7,387.8,362.0,318.0,274.0$, 255.8, 227.7, 199.8, 171.7, 134.8, 119.9, 108.9. IR (KBr) $v_{\max } / \mathrm{cm}^{-1}: 3338,3223,3151,2983,2930,2871,1645$, 1596, 1512, 1478, 1240, 1058, 826, 791. Anal. Calc. for $\mathrm{C}_{18} \mathrm{H}_{21} \mathrm{~N}_{5} \mathrm{O}_{5}: \mathrm{C}, 55.81 ; \mathrm{H}, 5.43 ; \mathrm{N}, 18.09$. Found: $\mathrm{C}, 55.72$; $\mathrm{H}, 5.51 ; \mathrm{N}, 18.13 \%$.

2-chloro-6-cyclohexylamino-9-( $\beta$-D-ribofuranosyl) purine $(8 a)$

White powder; mp $108-111^{\circ} \mathrm{C}$ (lit. 108-111 ${ }^{\circ} \mathrm{C}$ ).${ }^{6}{ }^{1} \mathrm{H}$ NMR (DMSO- $\left.d_{6}\right) \delta$ 1.112-1.916 (m, 10H, H cyclohexyl), 3.609 (m, 2H, H-5'), 3.946 (m, 1H, H-4'), 4.007 (s, 1H, H-1 cyclohexyl), 4.129 (m, 1H, H-3'), 4.510 (m, 1H, H2'), 5.822 (d, 1H, J 6.4 Hz, H-1'), 8.193 (d, 1H, J 8.8 Hz, NH), 8.377 (s, 1H, H-8).

2-chloro-6-cyclohexylamino-9-( $\beta-D-2,3,5-O$-triacetylribofuranosyl) purine ( $\left.8 \boldsymbol{a}^{\prime}\right)$

White needle crystal; mp 186-188 ${ }^{\circ} \mathrm{C} .{ }^{1} \mathrm{H}$ NMR $\left(\mathrm{DMSO}-d_{6}\right) \delta$ 1.089-1.830 (m, 10H, H cyclohexyl), 1.864 $\left(\mathrm{s}, 3 \mathrm{H}, \mathrm{COCH}_{3}\right), 1.925\left(\mathrm{~s}, 3 \mathrm{H}, \mathrm{COCH}_{3}\right), 2.028(\mathrm{~s}, 3 \mathrm{H}$, $\mathrm{COCH}_{3}$ ), 3.995 (s, 1H, H-1 cyclohexyl), 4.084 (m, 2H, H-5'), 4.183 (m, 1H, H-4'), 4.307 (m, 1H, H-3'), 4.575 (m, 1H, H-2'), 5.847 (d, 1H, J 4.8 Hz, H-1'), 8.198 (d, 1H, $J 8.4 \mathrm{~Hz}, \mathrm{NH}), 8.338$ (s, 1H, H-8). ${ }^{13} \mathrm{C}$ NMR (DMSO- $d_{6}$ ) $\delta 21.02$ (COCH3), 23.19 (COCH3), 25.04, 25.36, 25.56, 25.69, 32.96, 33.44 (C cyclohexy), 64.23 (5'-C), 70.64 (3'-C), 73.43 (2'-C), 82.12 (4'-C), 88.11 (1'-C), 118.85 (5C), 140.10 (8-C), 149.96 (6-C), 153.84 (4-C), 154.68 (2C), 168.42 (COCH3), 170.59 ( $\mathrm{COCH} 3)$.

2-chloro-6-cyclohexylamino-9-[(2-hydroxylethoxy) methyl]purine $(\mathbf{9 a})$

White powder; mp $165-167{ }^{\circ} \mathrm{C} .{ }^{1} \mathrm{H}$ NMR (DMSO- $d_{6}$ ) $\delta$ 1.107-1.922 (m, 10H, H cyclohexyl), 3.493 (m, 4H, $\mathrm{HOCH}_{2} \mathrm{CH}_{2} \mathrm{O}$ ), 4.004 (br, 1H, H cyclohexyl), 5.518 (s, $2 \mathrm{H}, \mathrm{NCH}_{2} \mathrm{O}$ ), 8.154 (d, 1H, J 7.2 Hz, NH), 8.283 (s, 1H, H-8). ${ }^{13} \mathrm{C}$ NMR (DMSO- $d_{6}$ ) $\delta 33.48,32.47,25.56,25.35$ (C cyclohexyl), 49.47 (NCH), $60.34\left(\mathrm{HOCH}_{2} \mathrm{CH}_{2} \mathrm{O}\right), 71.23$ $\left(\mathrm{HOCH}_{2} \mathrm{CH}_{2} \mathrm{O}\right), 72.92\left(\mathrm{NCH}_{2} \mathrm{O}\right), 118.33(5-\mathrm{C}), 141.77$ (8C), 150.41 (6-C), 154.08 (4-C), 154.70 (2-C). MS (ESI) $\mathrm{m} / \mathrm{z}$ [ $\left[\mathrm{M}^{+} \mathrm{Na}^{+}-1,347.7\right], 325.8,311.6,284.0,228.8,101.9$. IR (KBr) $v_{\text {max }} / \mathrm{cm}^{-1}: 3441,3236,2937,2923,2857,1621$, 1310, 1216. Anal. Calc. for $\mathrm{C}_{14} \mathrm{H}_{20} \mathrm{ClN}_{5} \mathrm{O}_{2}$ : C, 51.61; $\mathrm{H}$, 6.14; N, 21.51. Found: C, 51.47; H, 6.18; N, $21.63 \%$. 


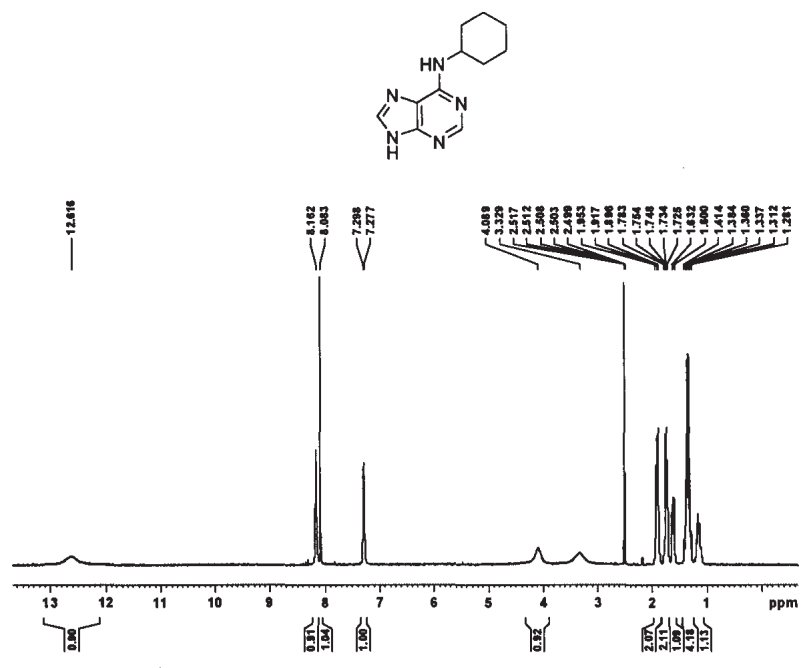

Figure S1. ' $\mathrm{H}$ NMR of $1 \mathrm{a}$.
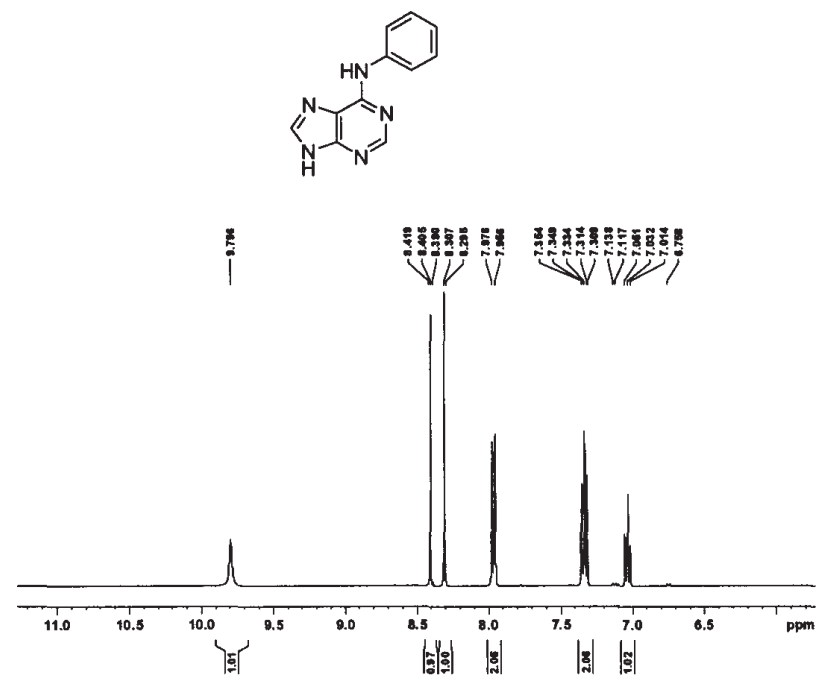

Figure S2. ${ }^{1} \mathrm{H}$ NMR of $\mathbf{1 b}$.
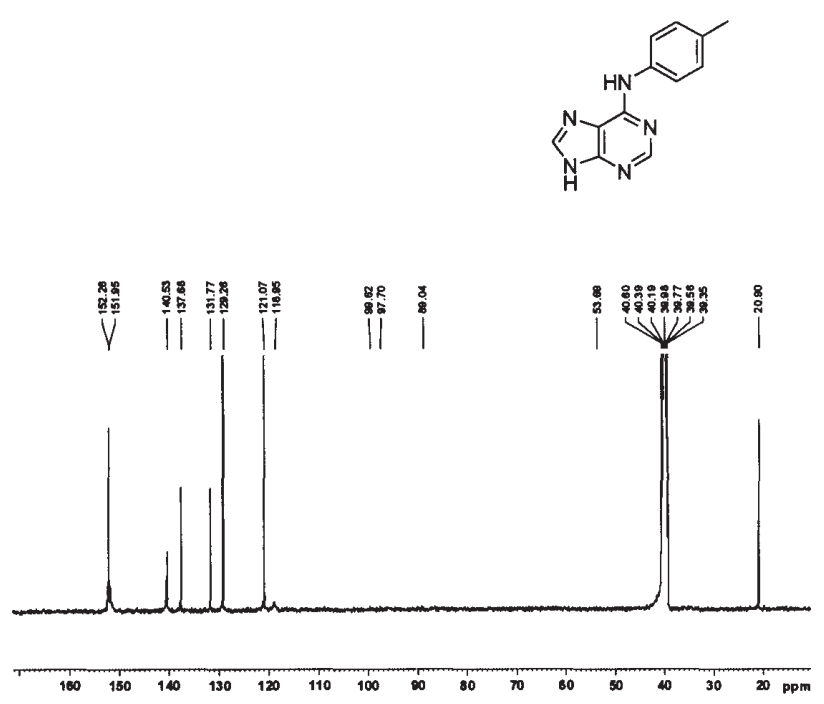

Figure S5. ${ }^{13} \mathrm{C}$ NMR of $1 \mathrm{c}$.
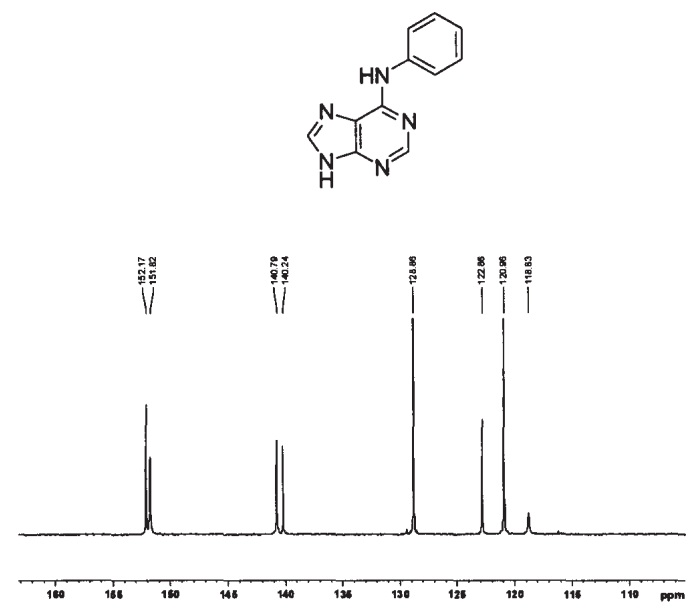

Figure S3. ${ }^{13} \mathrm{C}$ NMR of 1 b.
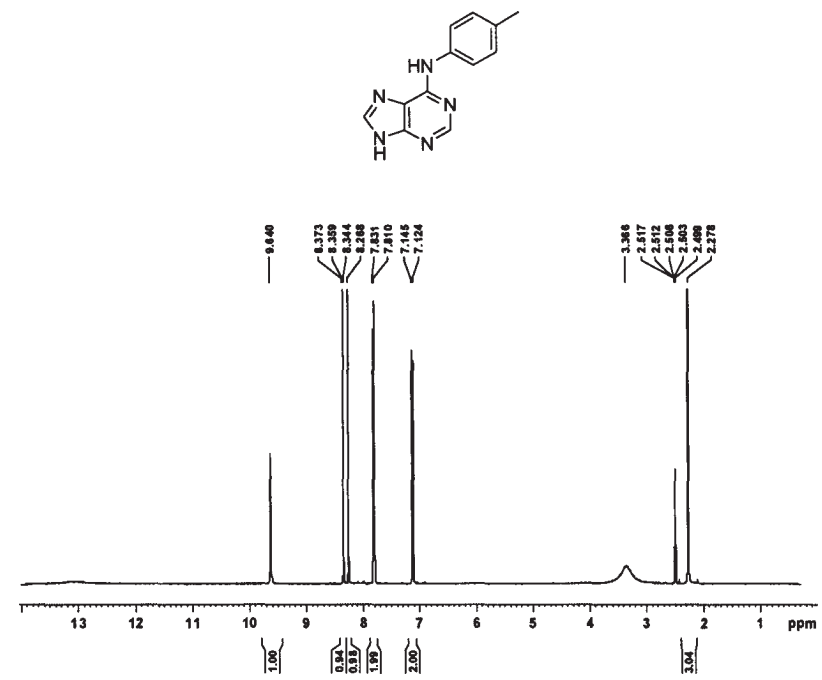

Figure S4. ${ }^{1} \mathrm{H}$ NMR of $1 \mathrm{c}$.
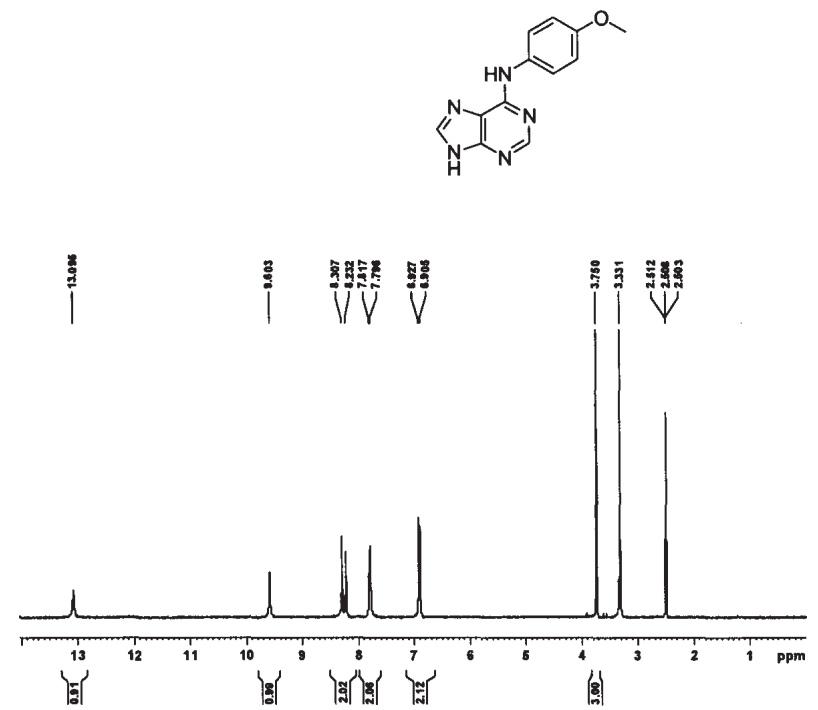

Figure S6. ${ }^{1} \mathrm{H}$ NMR of $1 \mathrm{~d}$. 

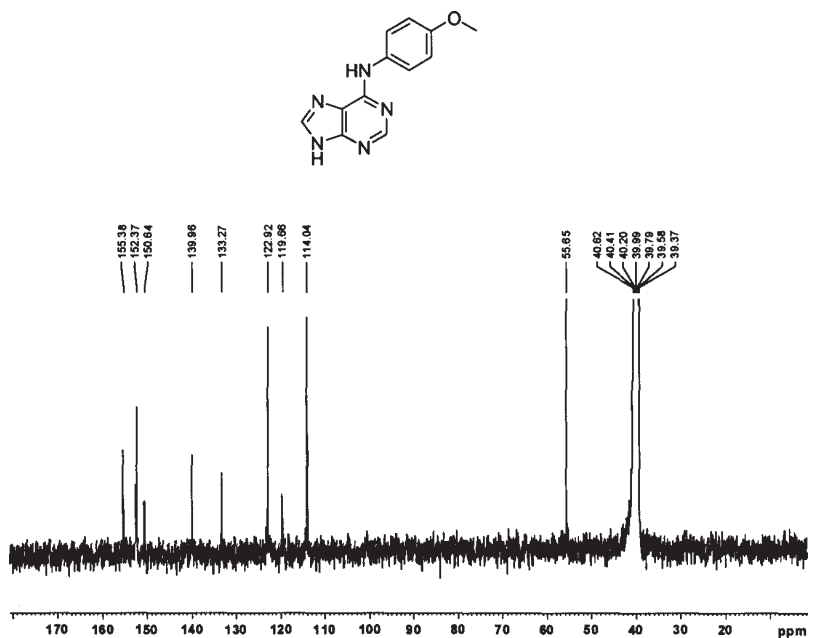

Figure S7. ${ }^{13} \mathrm{C}$ NMR of 1d.
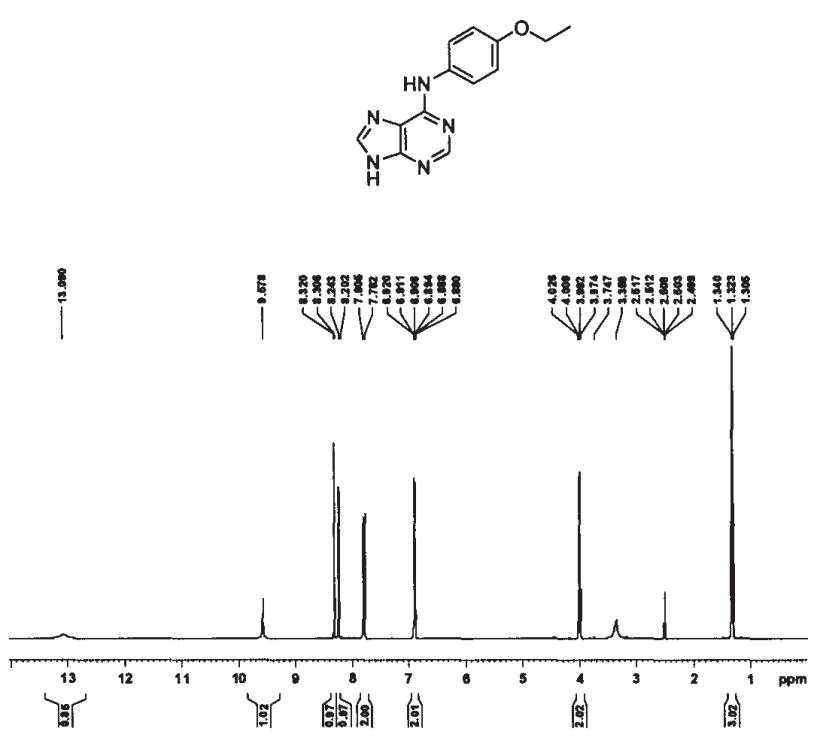

Figure S8. ${ }^{1} \mathrm{H}$ NMR of $1 \mathrm{e}$.
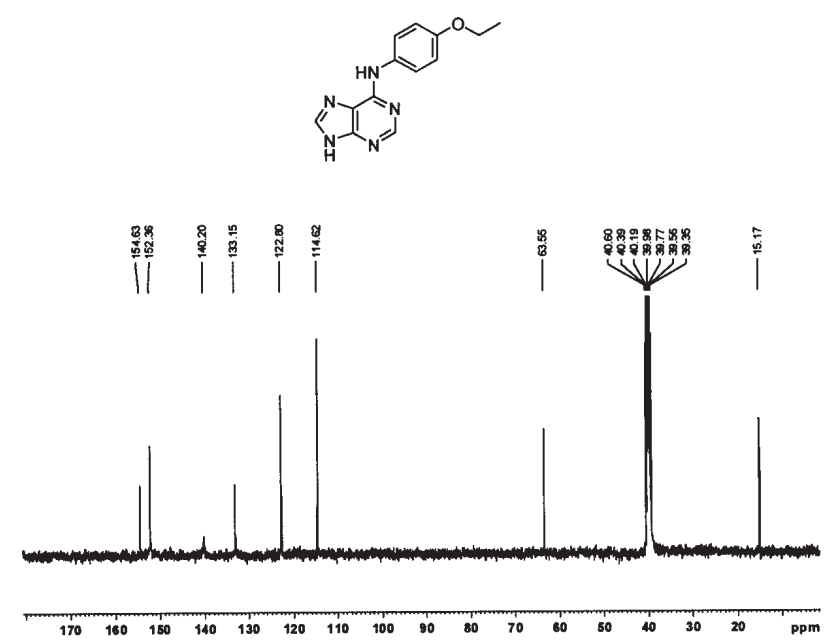

Figure S9. ${ }^{13} \mathrm{C}$ NMR of $1 \mathrm{e}$.
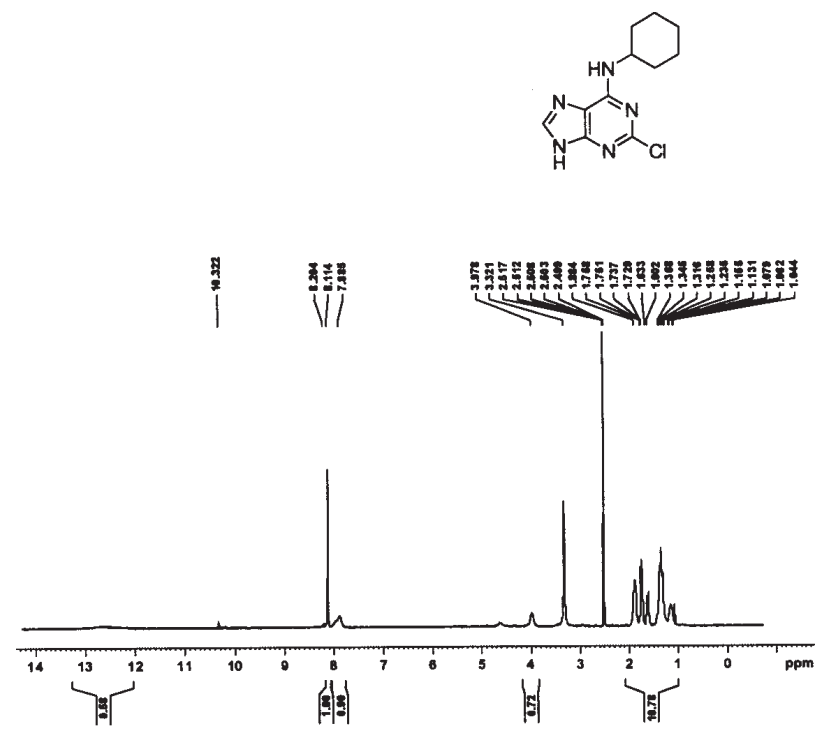

Figure S10. ${ }^{1} \mathrm{H}$ NMR of 2 a.
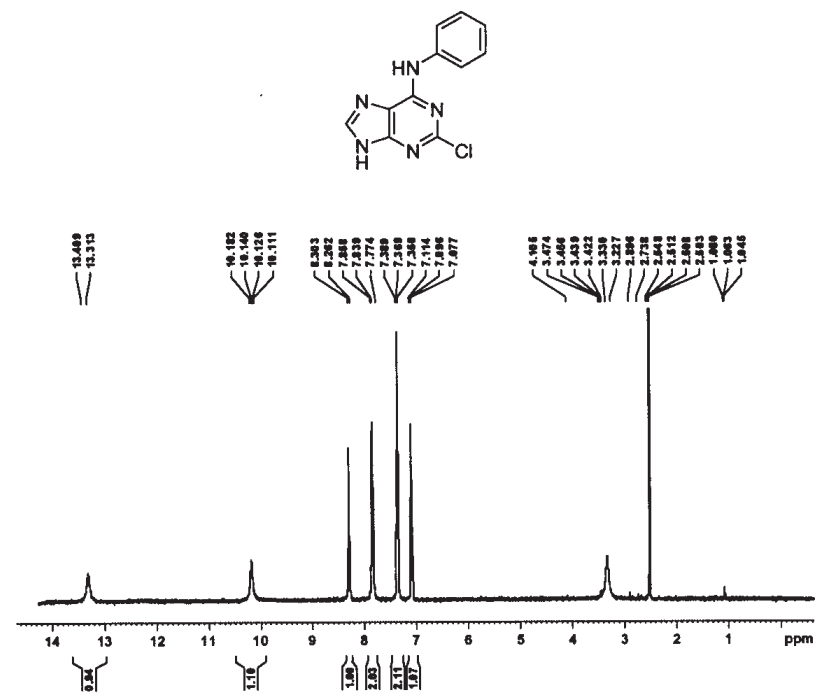

Figure S11. ${ }^{1} \mathrm{H}$ NMR of $\mathbf{2 b}$.
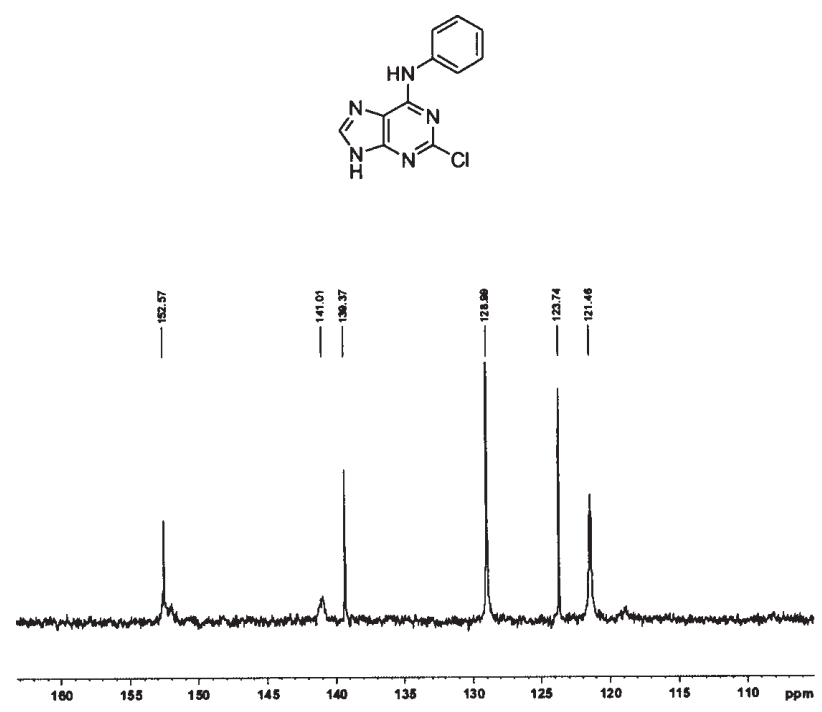

Figure $\mathrm{S12} .{ }^{13} \mathrm{C} \mathrm{NMR}$ of $2 \mathrm{~b}$. 


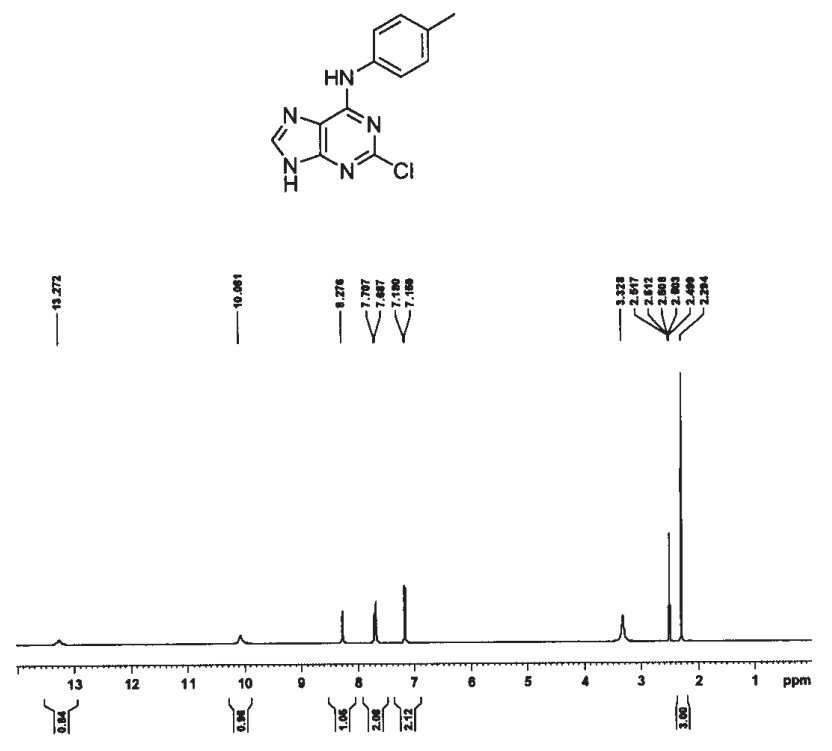

Figure S13. ${ }^{1} \mathrm{H}$ NMR of $2 \mathrm{c}$.
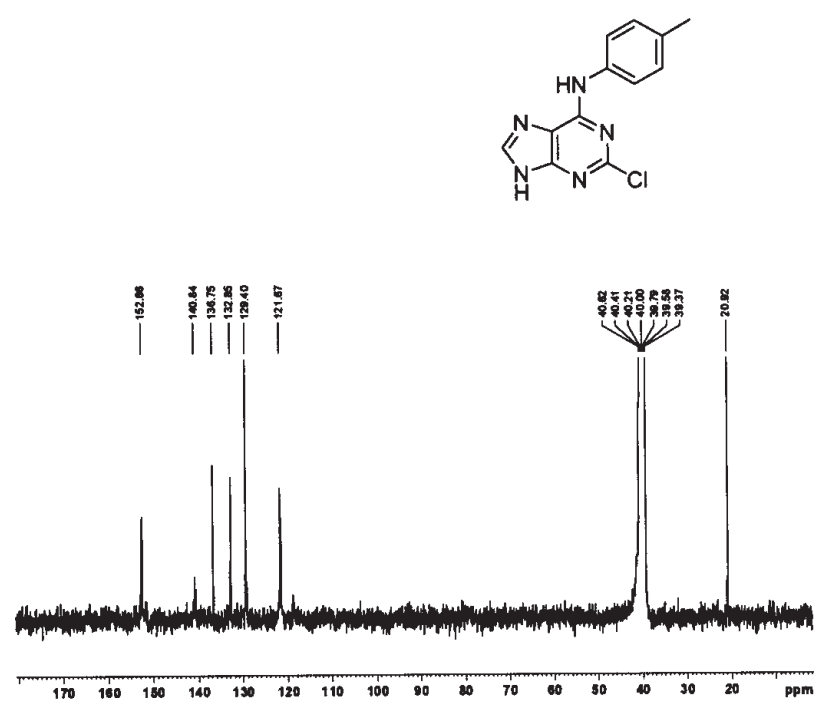

Figure S14. ${ }^{13} \mathrm{C}$ NMR of $2 \mathrm{c}$.

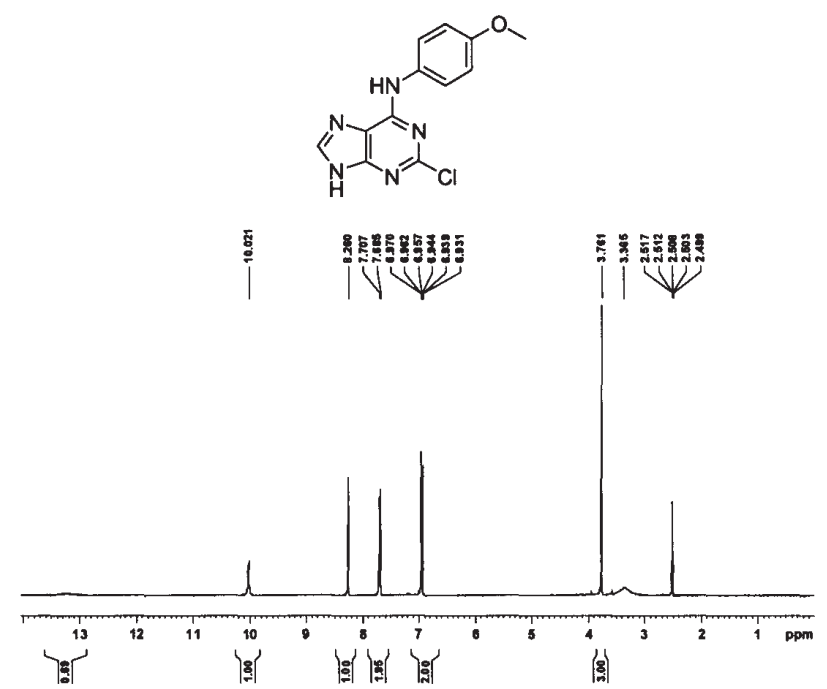

Figure S15. ${ }^{1} \mathrm{H}$ NMR of $\mathbf{2 d}$.

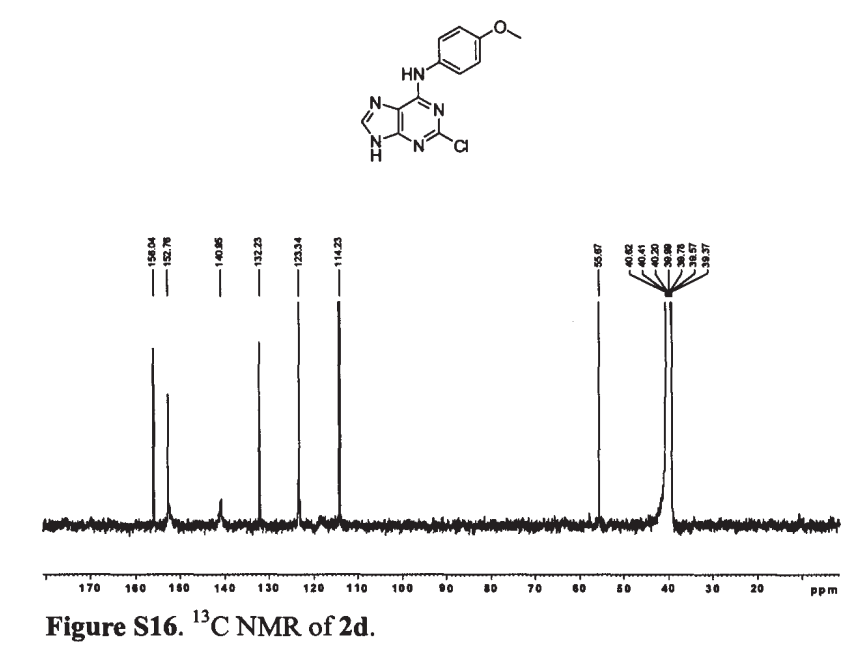

Figure S16. ${ }^{13} \mathrm{C}$ NMR of $2 d$.
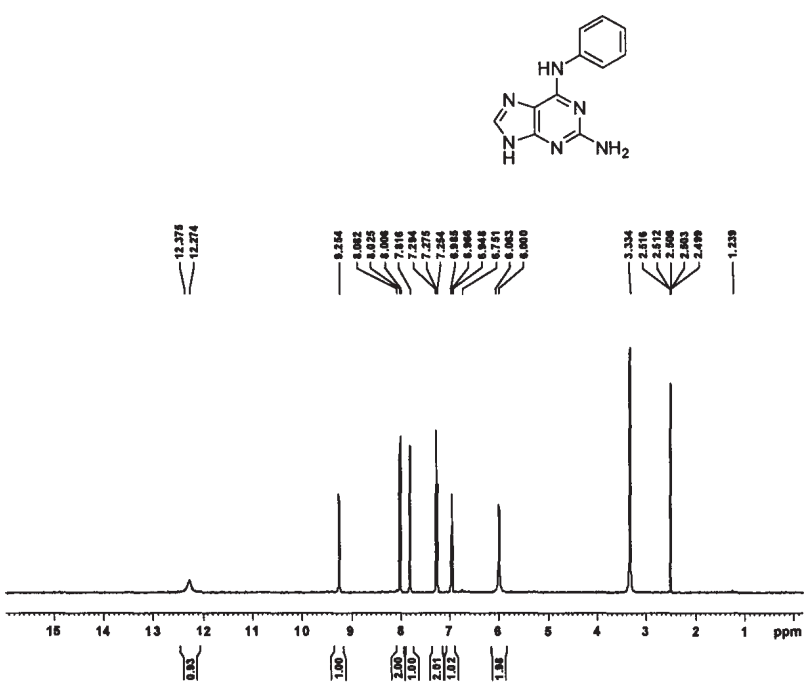

Figure S17. ${ }^{1} \mathrm{H}$ NMR of $\mathbf{3 b}$.
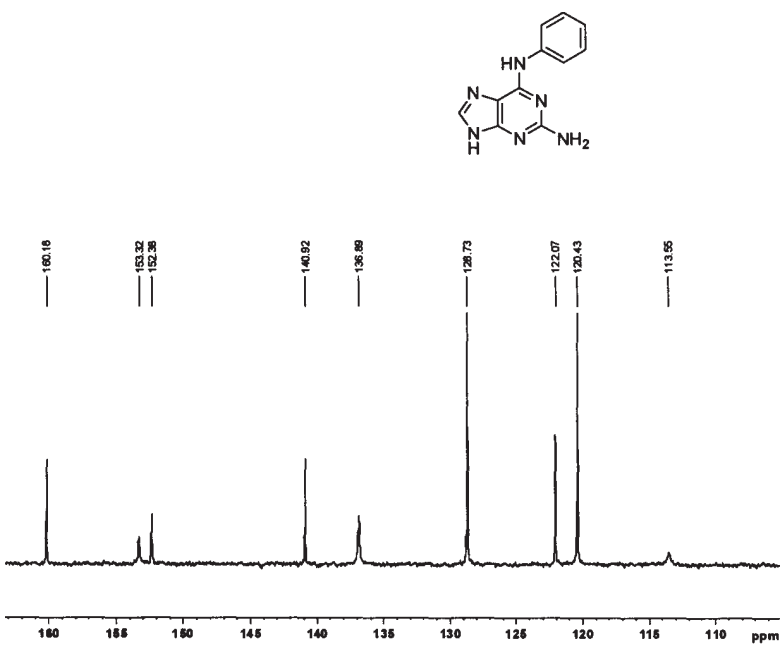

Figure S18. ${ }^{13} \mathrm{C}$ NMR of $3 b$. 


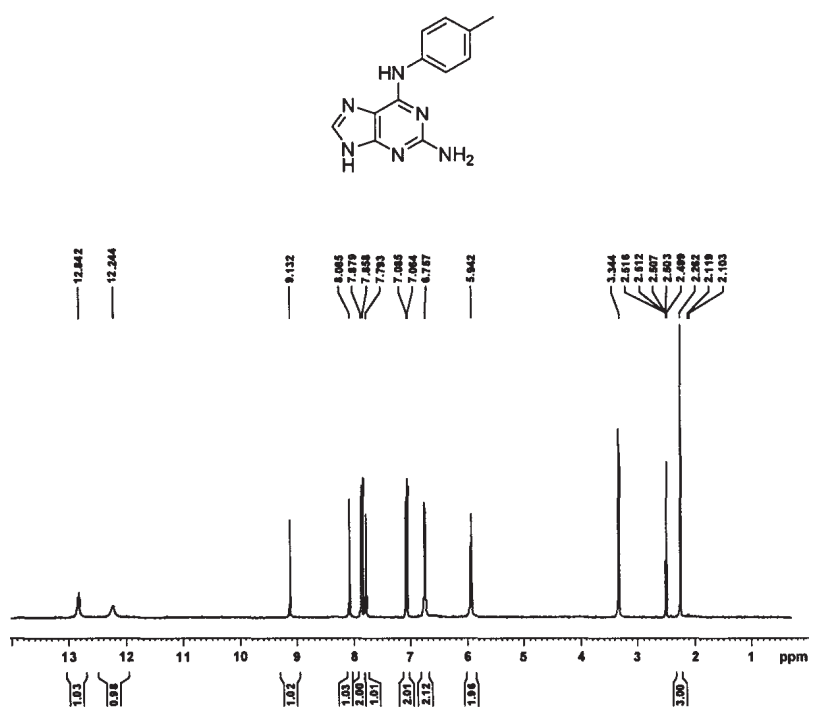

Figure S19. ${ }^{1} \mathrm{H}$ NMR of $3 \mathrm{c}$.
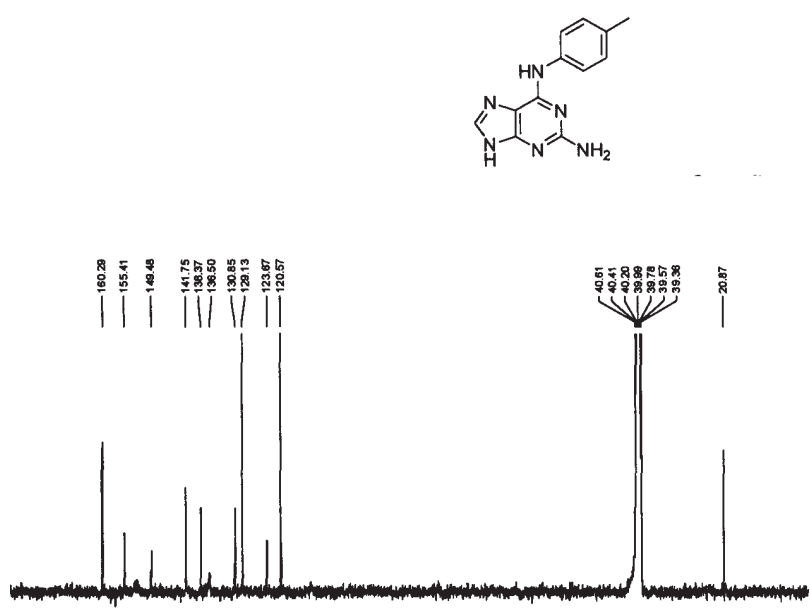

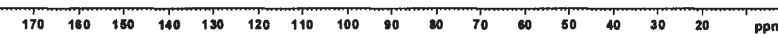
Figure S20. ${ }^{13} \mathrm{C}$ NMR of $3 \mathrm{c}$.

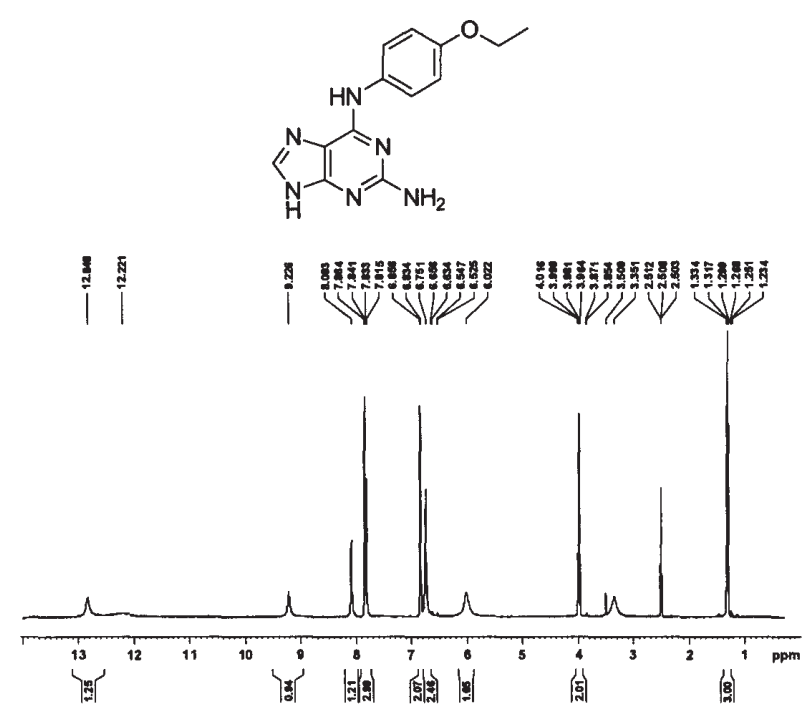

Figure S21. ${ }^{1} \mathrm{H}$ NMR of $3 e$.
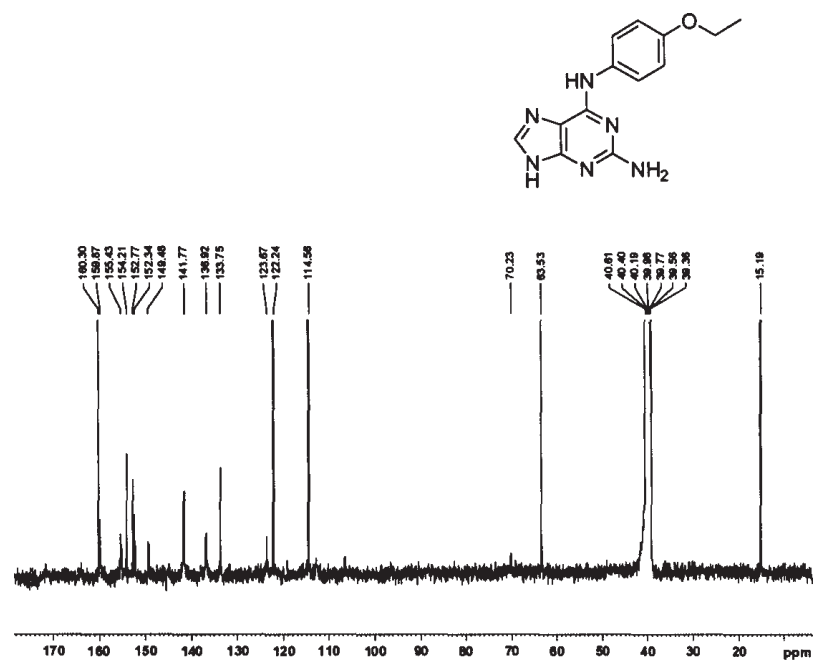

Figure S22. ${ }^{13} \mathrm{C}$ NMR of $3 e$.

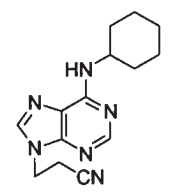

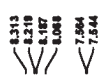
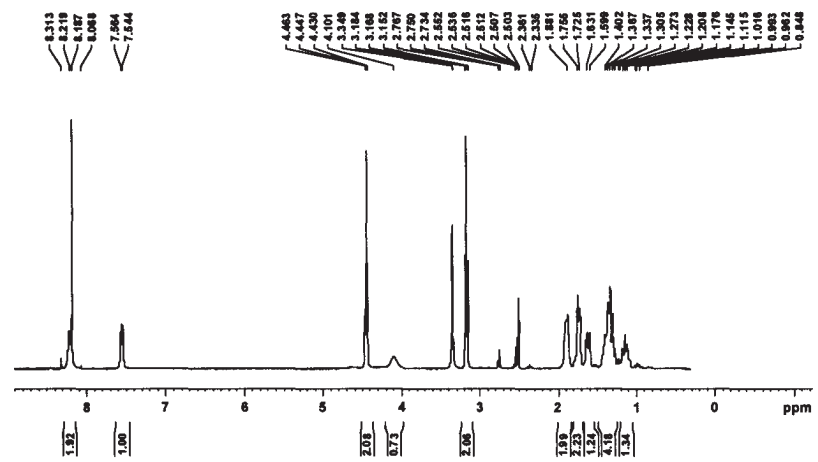

Figure S23. ${ }^{1} \mathrm{H}$ NMR of $4 a$.

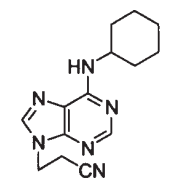

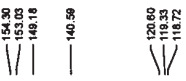
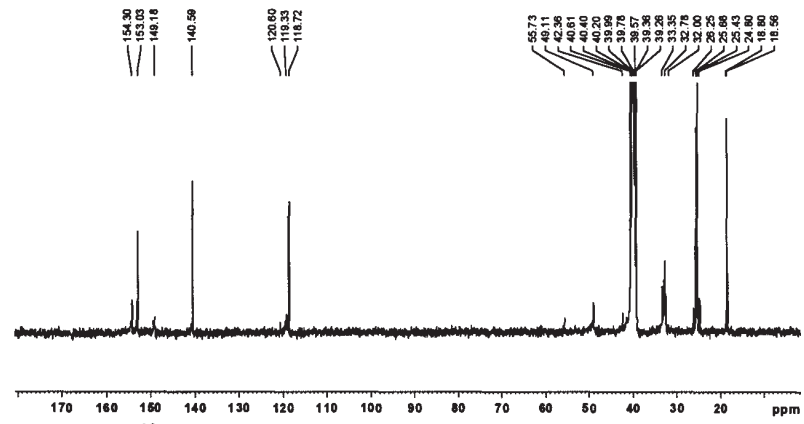
Figure S24. ${ }^{13} \mathrm{C}$ NMR of 4a. 


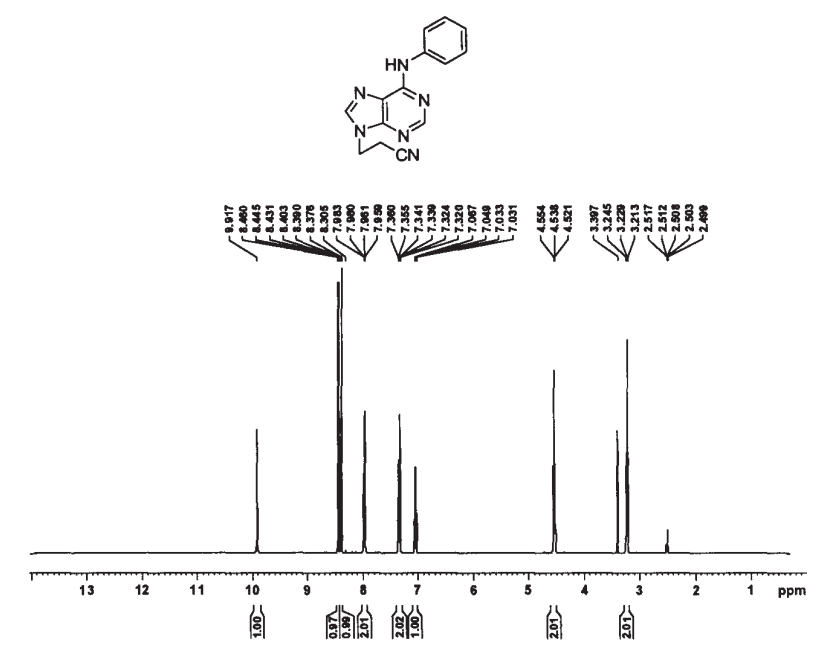

Figure S25. 'H NMR of $4 b$.
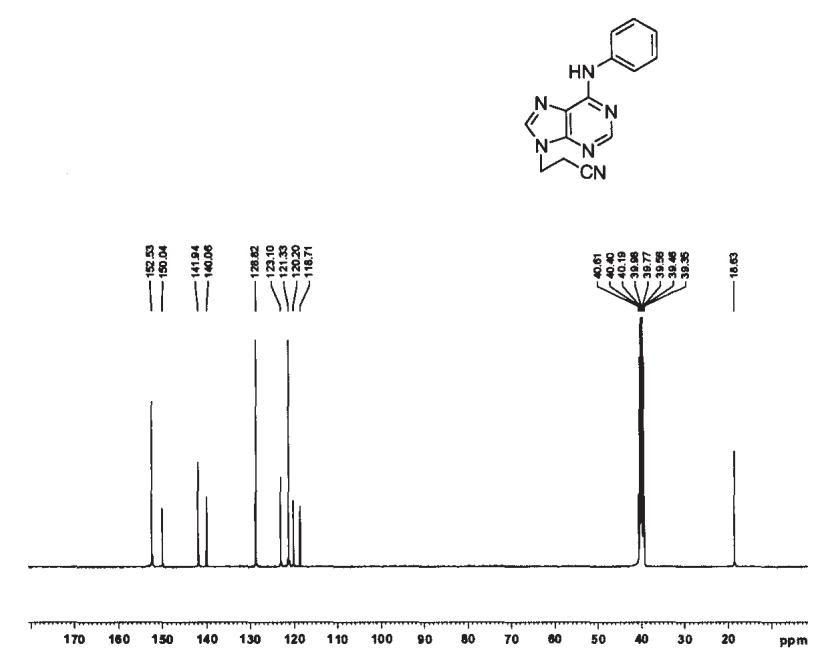

Figure $\mathbf{S 2 6}$. ${ }^{13} \mathrm{C}$ NMR of $4 \mathrm{~b}$.

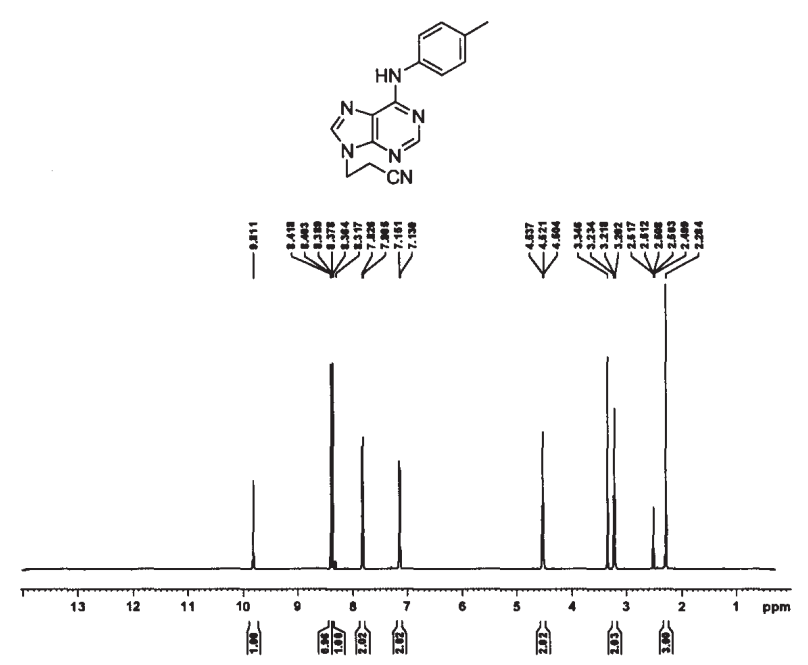

Figure S27. ${ }^{1} \mathrm{H}$ NMR of $4 c$.
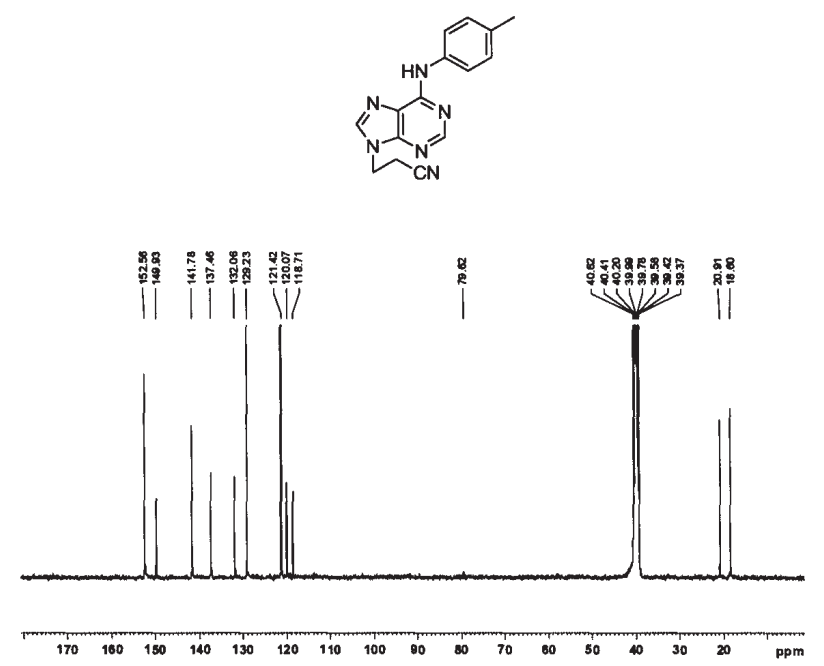

Figure S28. ${ }^{13} \mathrm{C}$ NMR of $4 \mathrm{c}$.
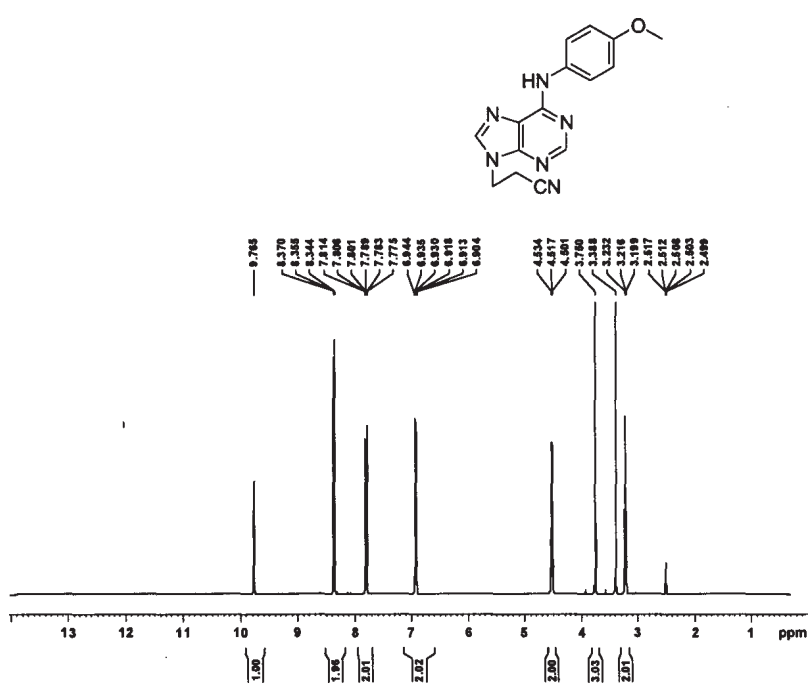

Figure S29. ${ }^{1} \mathrm{H}$ NMR of 4 d.

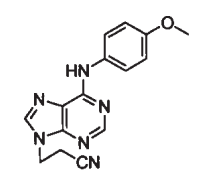

ลำ

IVI I IVI

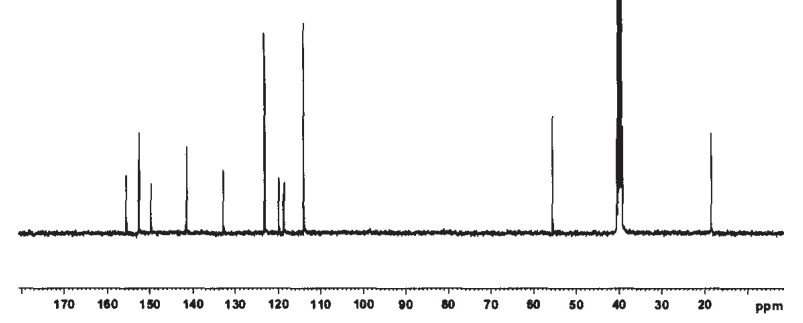

Figure S30. ${ }^{13} \mathrm{C}$ NMR of $4 d$. 


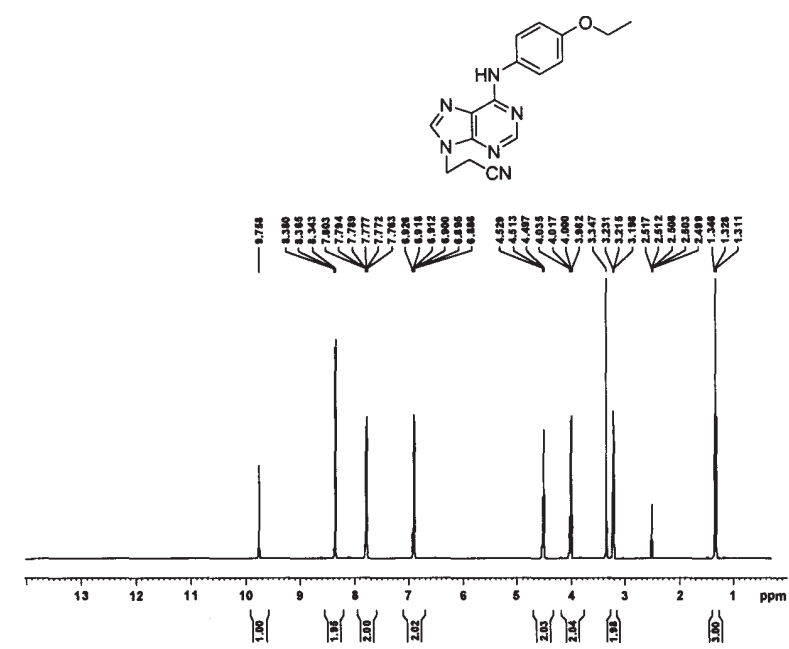

Figure S31. ${ }^{1} \mathrm{H}$ NMR of $4 e$.

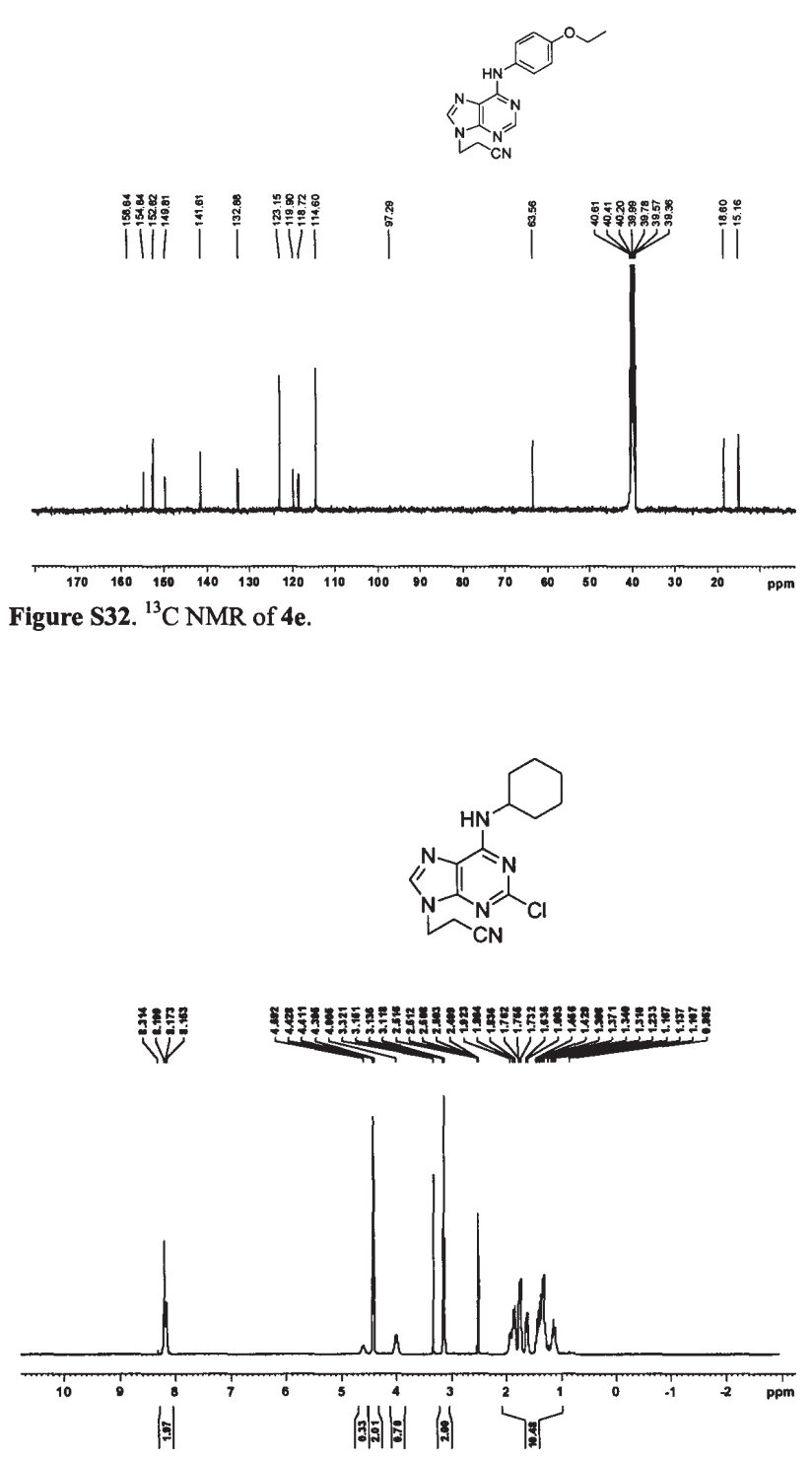

Figure S33. ${ }^{1} \mathrm{H}$ NMR of 5 a.

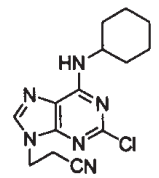

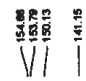

$\stackrel{n}{V}$

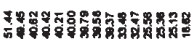

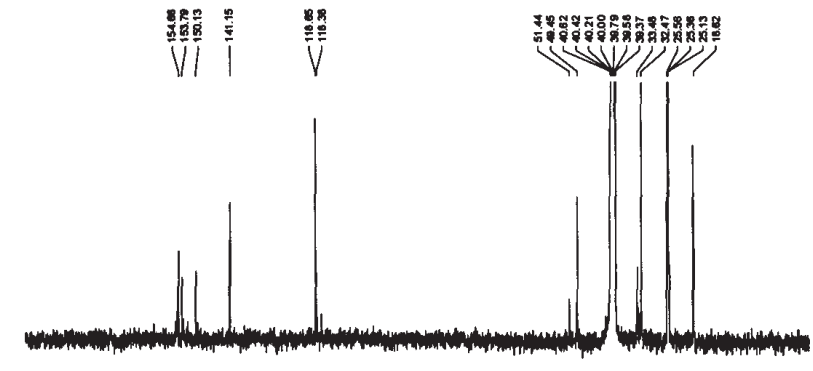

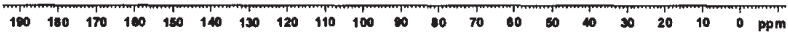
Figure S34. ${ }^{13} \mathrm{C}$ NMR of $5 a$.
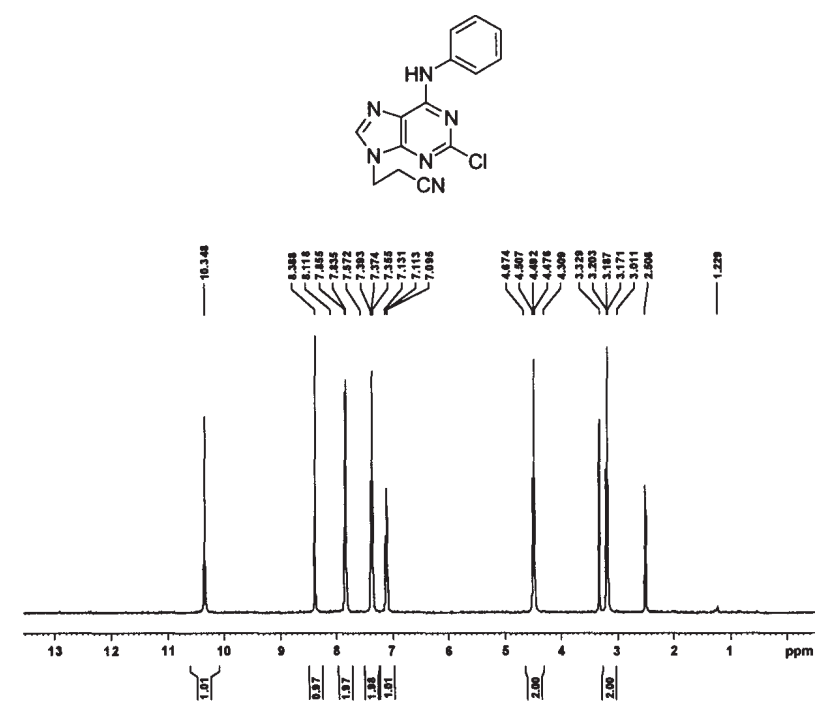

Figure S35. ${ }^{1} \mathrm{H}$ NMR of $\mathbf{5 b}$.
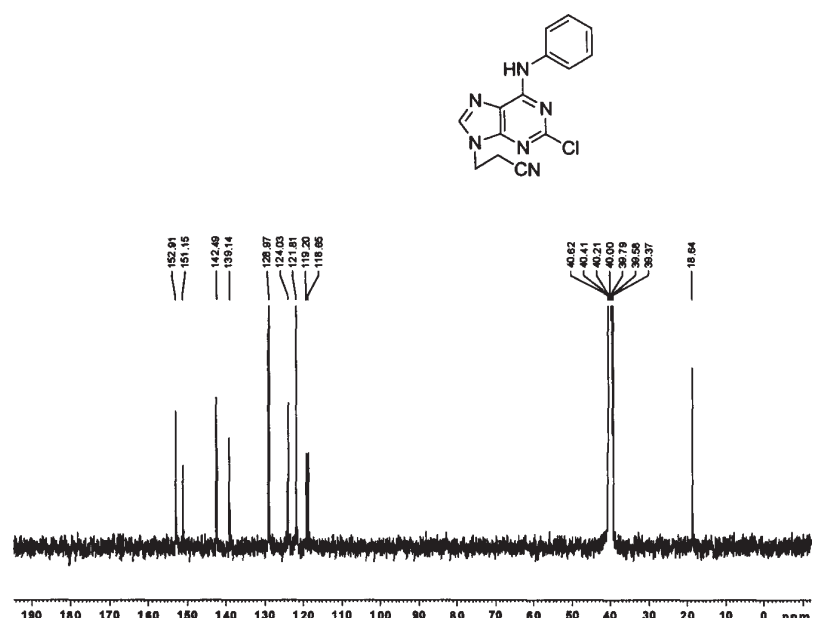

Figure S36. ${ }^{13} \mathrm{C}$ NMR of 5 b. 


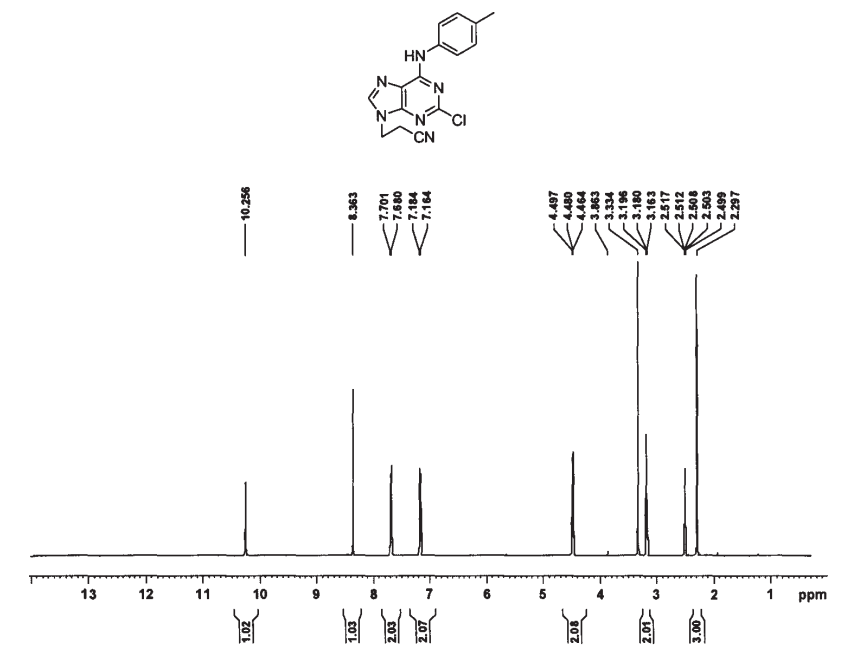

Figure S37. ${ }^{1} \mathrm{H}$ NMR of 5c.
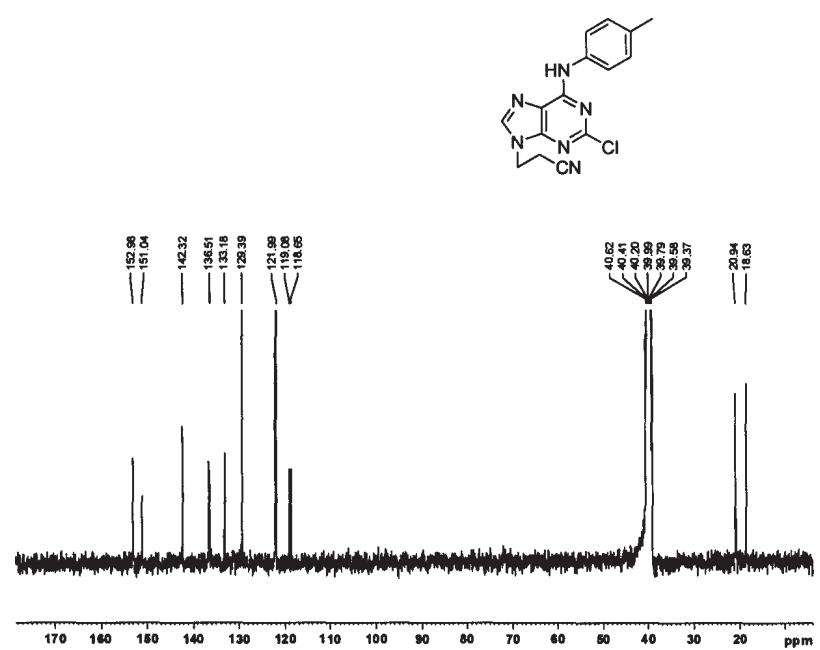

Figure S38. ${ }^{13} \mathrm{C}$ NMR of $5 c$.

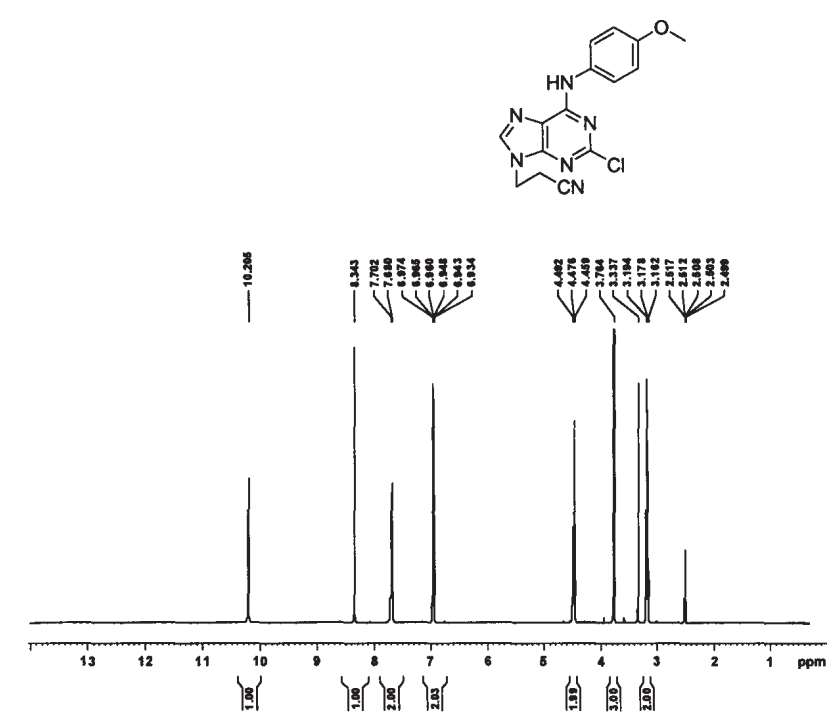

Figure S39. ' $\mathrm{H}$ NMR of $5 d$.

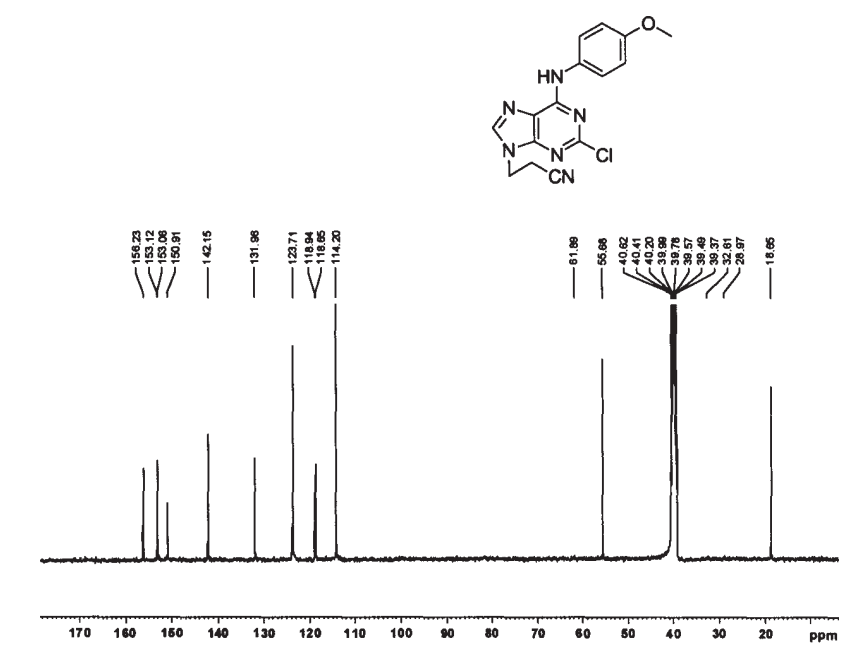

Figure S40. ${ }^{13} \mathrm{C}$ NMR of 5d.

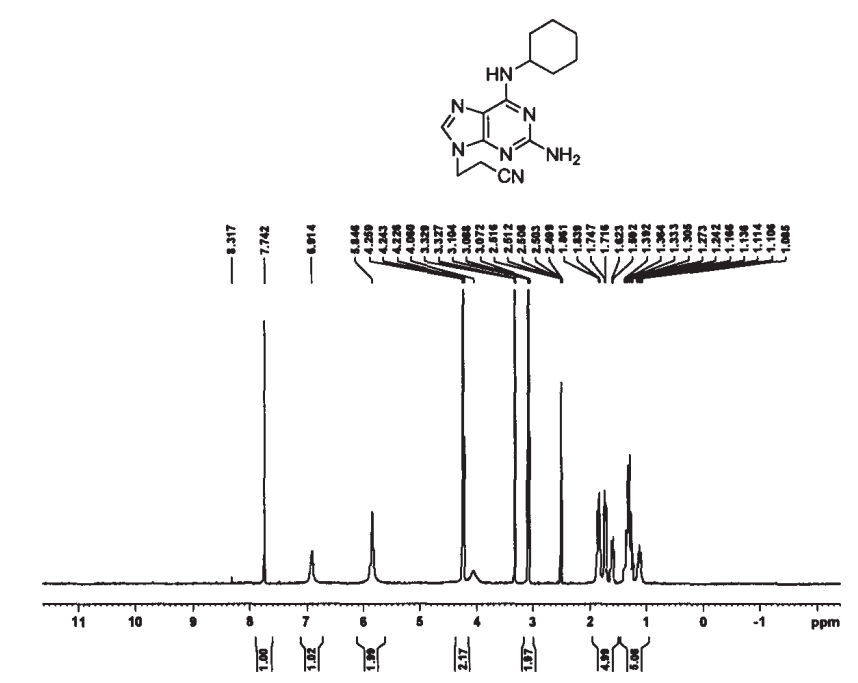

Figure S41. ${ }^{1} \mathrm{H}$ NMR of $6 a$.
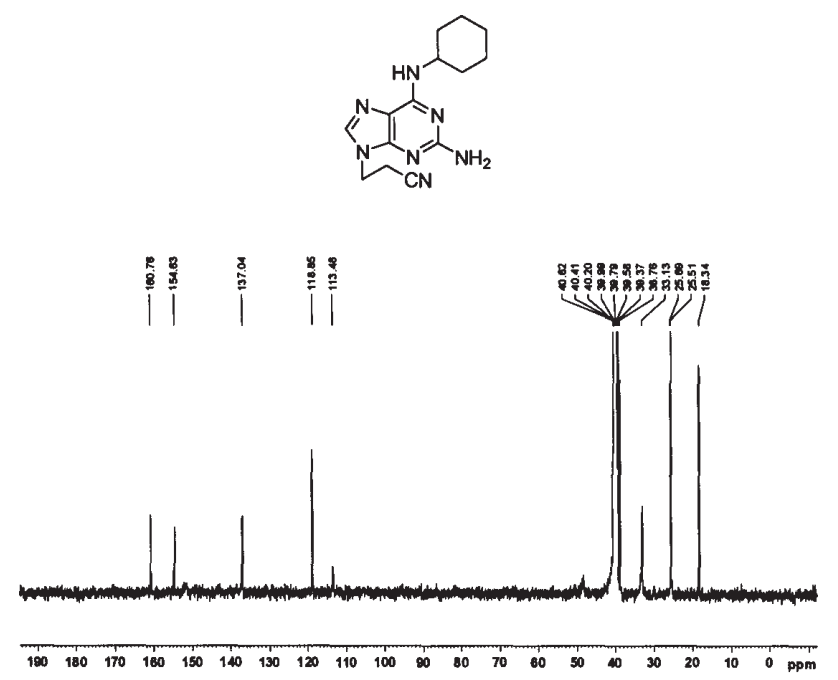

Figure S42. ${ }^{13} \mathrm{C}$ NMR of 6 a. 


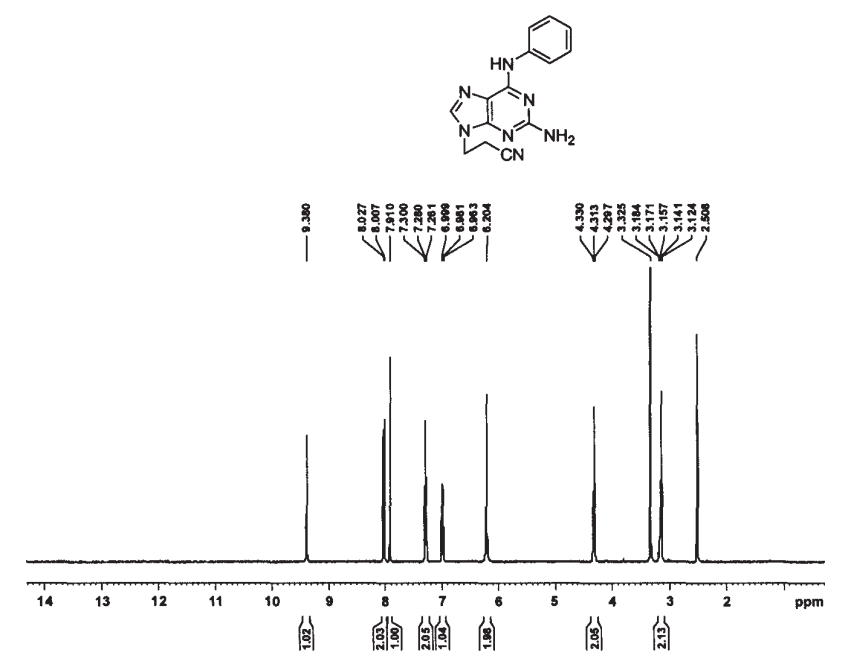

Figure S43. ${ }^{1} \mathrm{H}$ NMR of $6 \mathrm{~b}$.

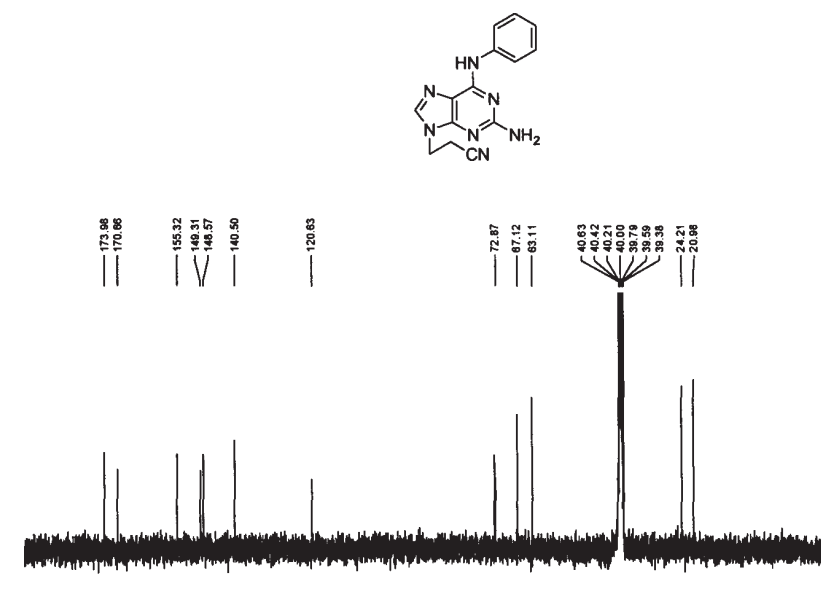

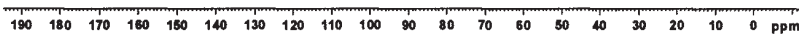
Figure $S 44 .{ }^{13} \mathrm{C}$ NMR of $6 \mathrm{~b}$.
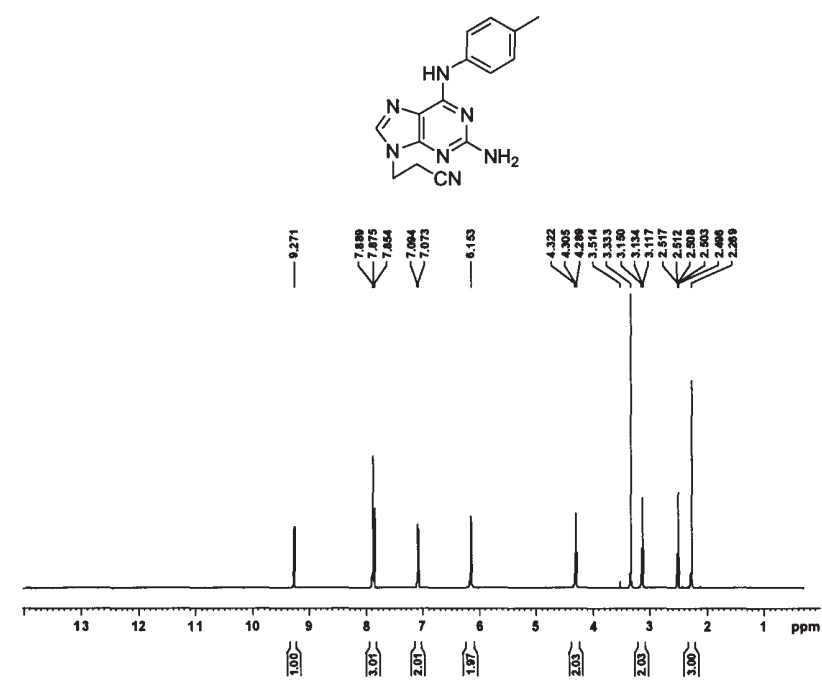

Figure S45. ${ }^{1} \mathrm{H}$ NMR of $6 c$.
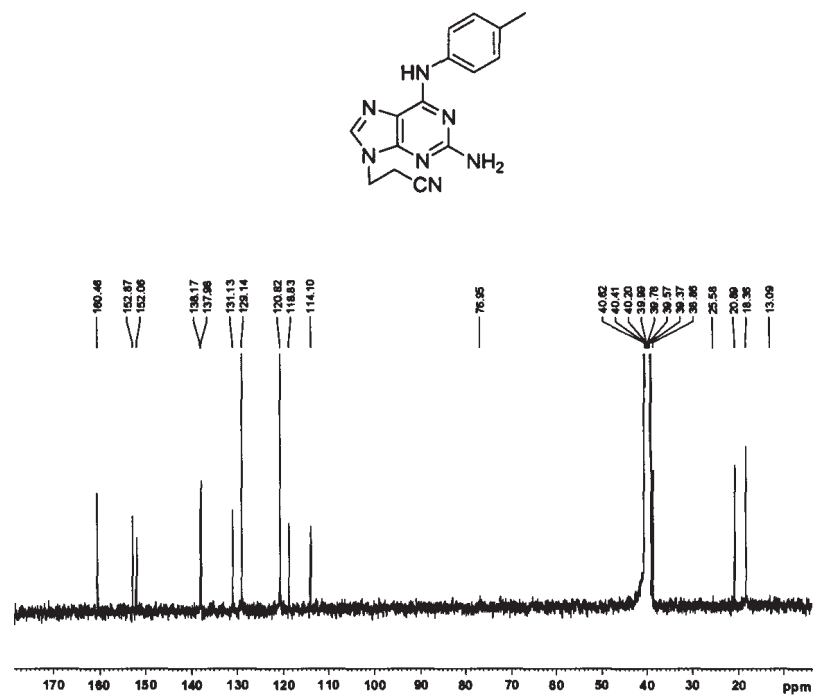

Figure S46. ${ }^{13} \mathrm{C}$ NMR of $6 \mathrm{c}$.

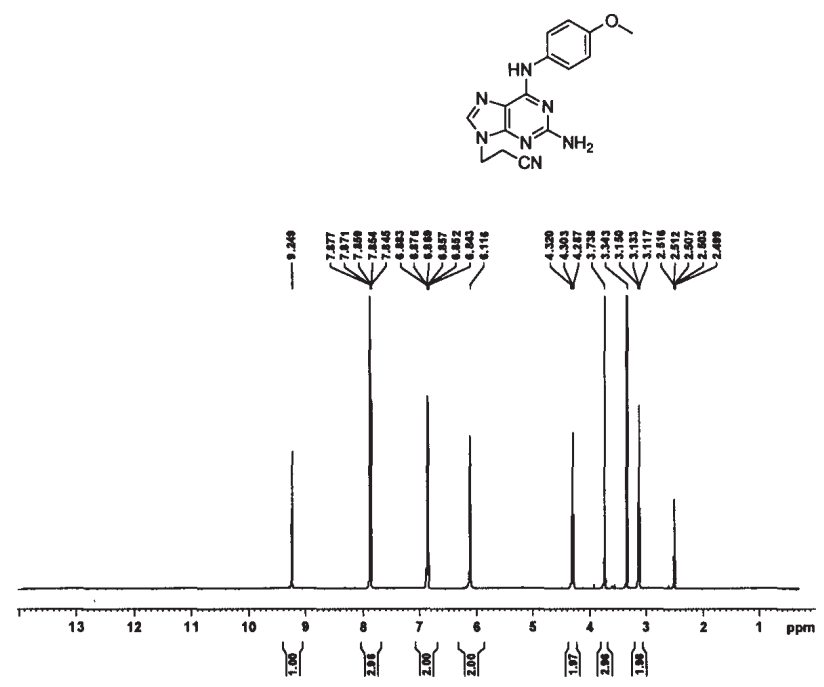

Figure S47. ${ }^{1} \mathrm{H}$ NMR of 6 d.
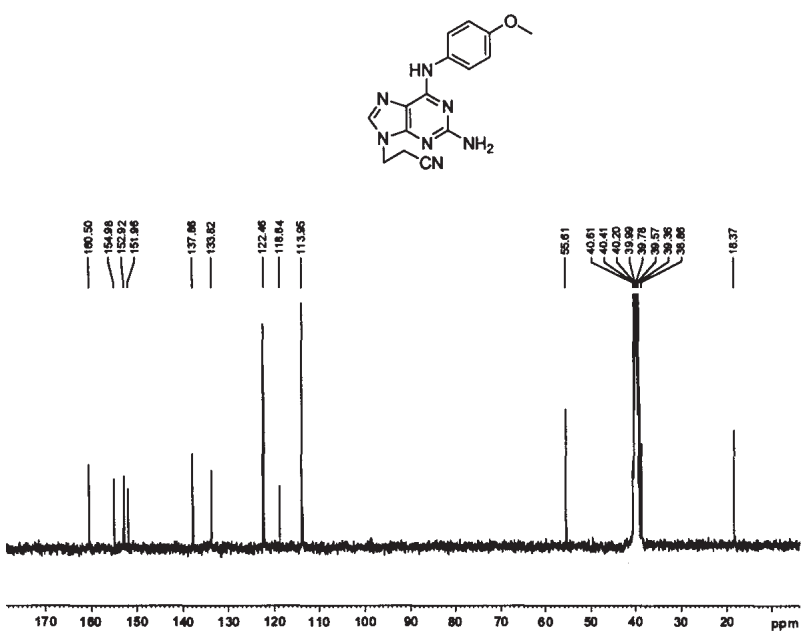

Figure S48. ${ }^{13} \mathrm{C}$ NMR of $6 \mathrm{~d}$. 


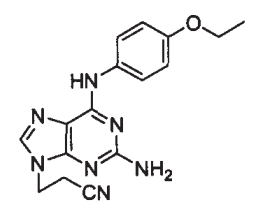

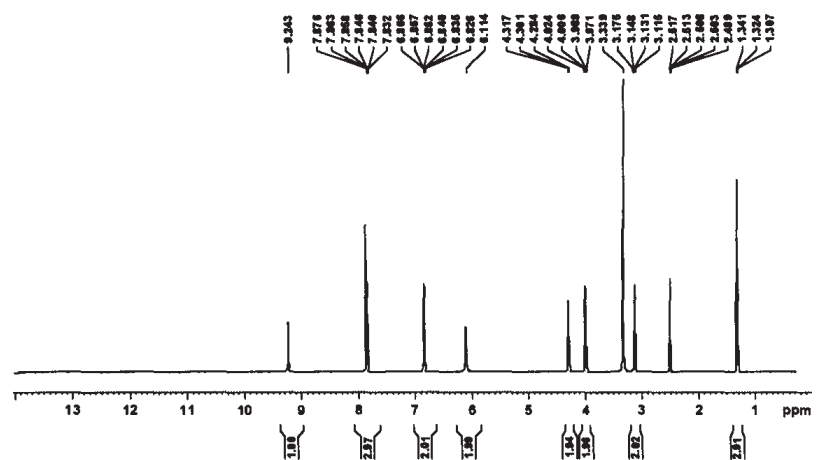

Figure S49. ${ }^{1} \mathrm{H}$ NMR of $6 e$.
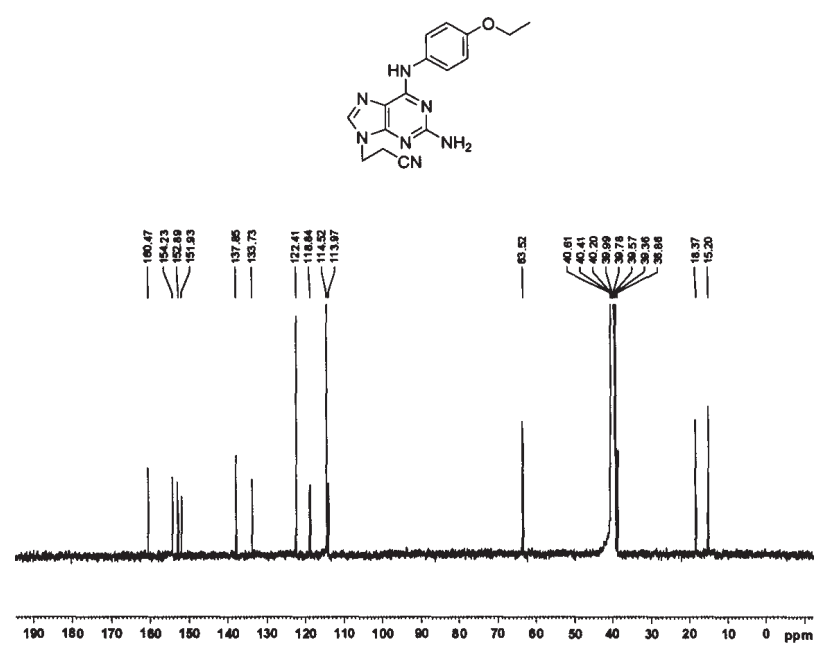

Figure S50. ${ }^{13} \mathrm{C}$ NMR of $6 \mathrm{e}$.

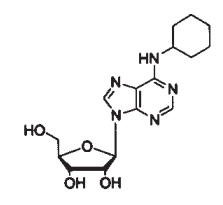

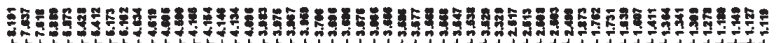

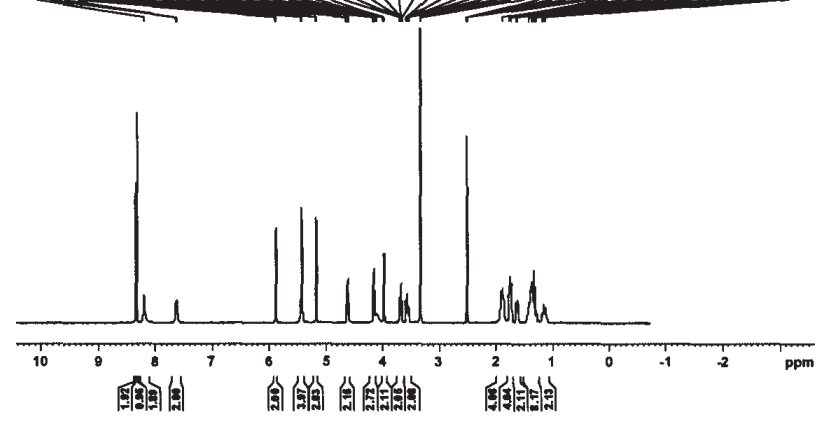

Figure S51. ${ }^{1} \mathrm{H}$ NMR of 7a.

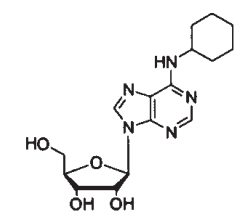

VII|

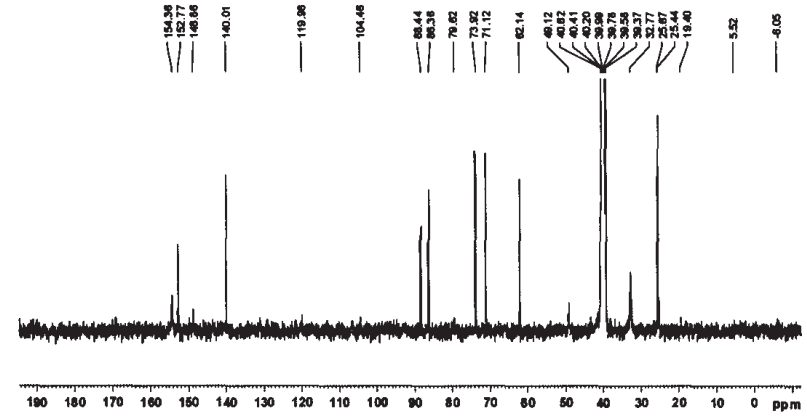

Figure S52. ${ }^{13} \mathrm{C}$ NMR of 7 a.
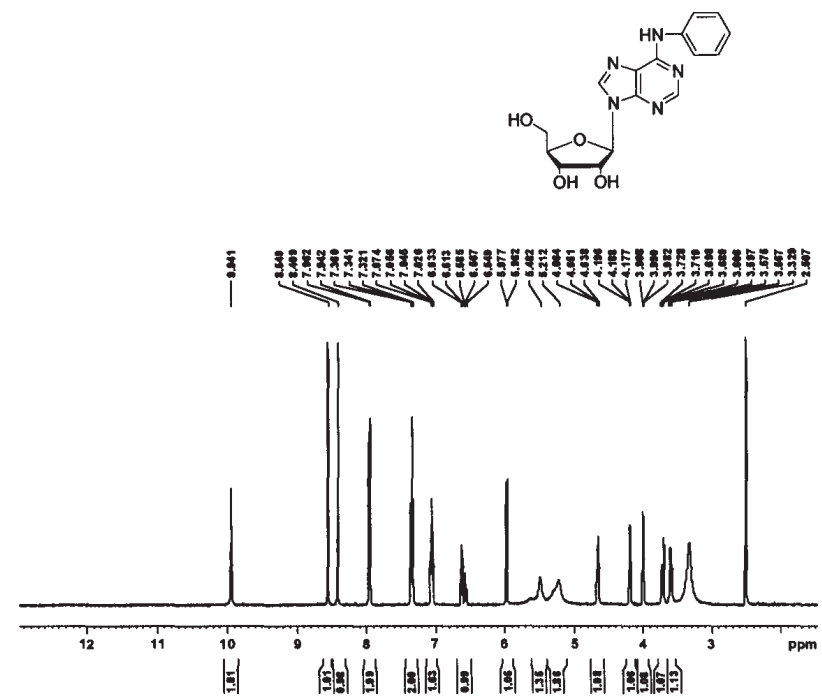

Figure S53. ${ }^{1} \mathrm{H}$ NMR of $7 \mathbf{b}$.
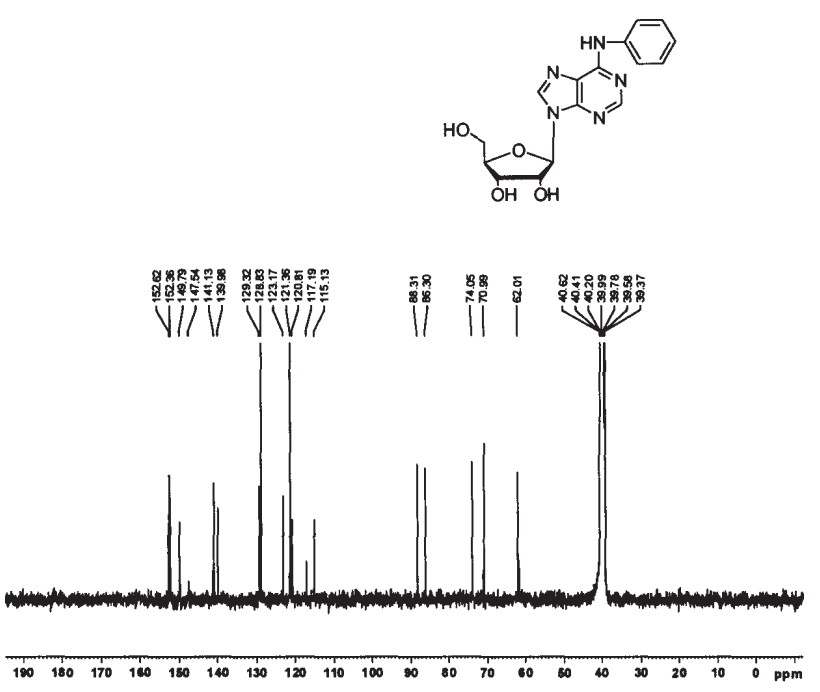

Figure S54. ${ }^{13} \mathrm{C}$ NMR of $7 \mathbf{b}$. 

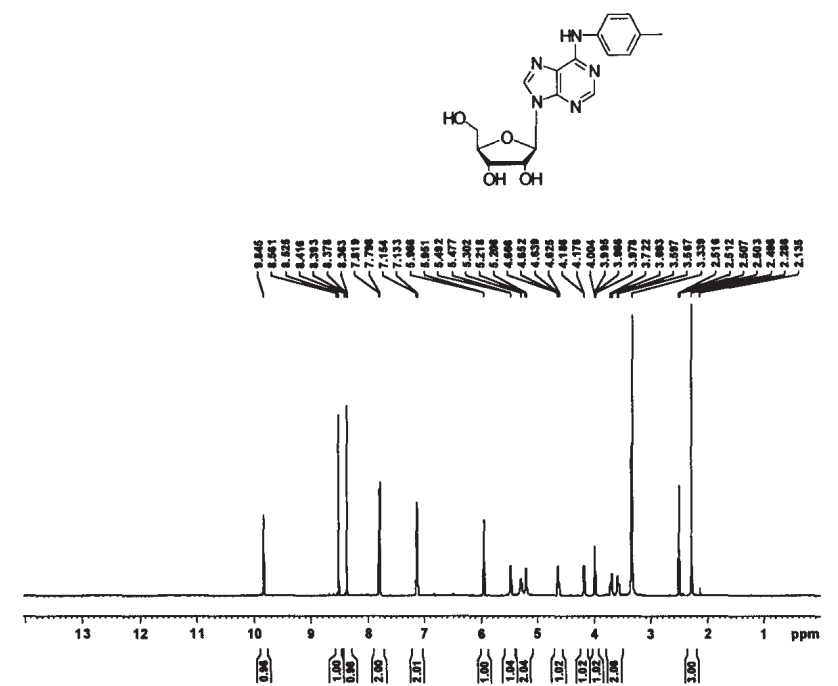

Figure S55. ${ }^{1} \mathrm{H}$ NMR of $7 \mathrm{c}$.
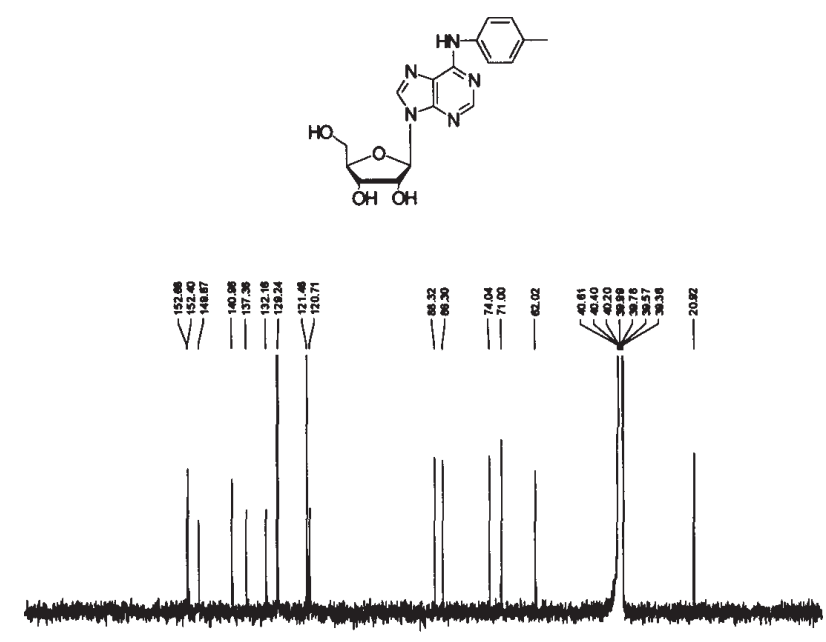

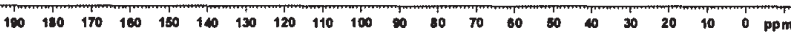

Figure S56. ${ }^{13} \mathrm{C}$ NMR of $7 \mathrm{c}$.

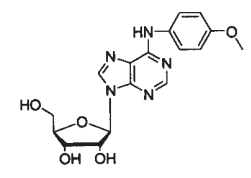

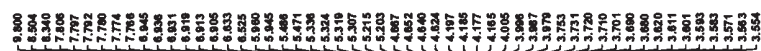

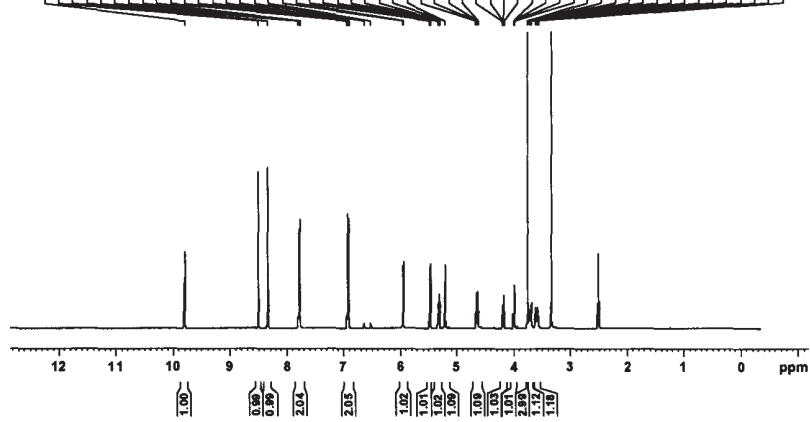

Figure S57. ${ }^{1} \mathrm{H}$ NMR of $7 \mathrm{~d}$.

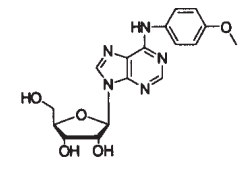

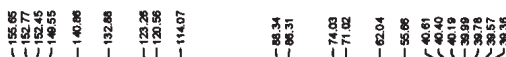

IVI

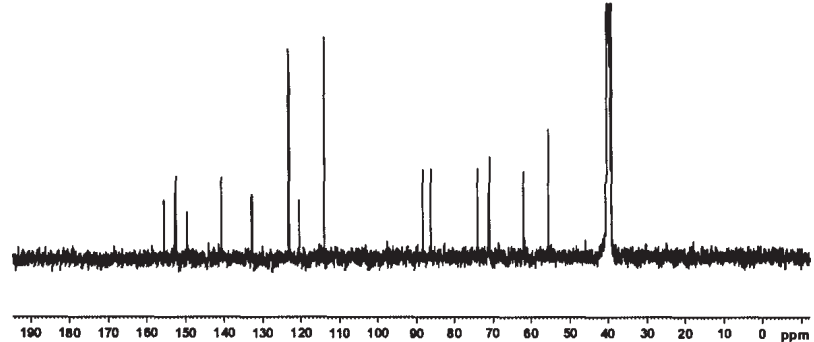

Figure $558 .{ }^{13} \mathrm{C}$ NMR of $7 \mathrm{~d}$.

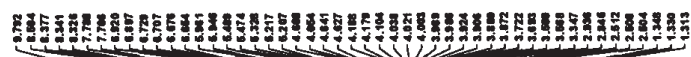

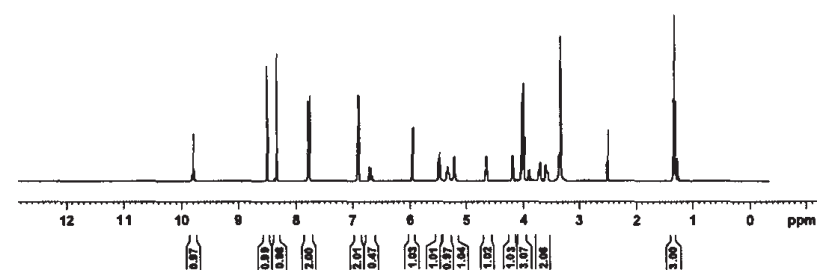

Figure S59. ${ }^{1} \mathrm{H}$ NMR of $7 \mathrm{e}$.
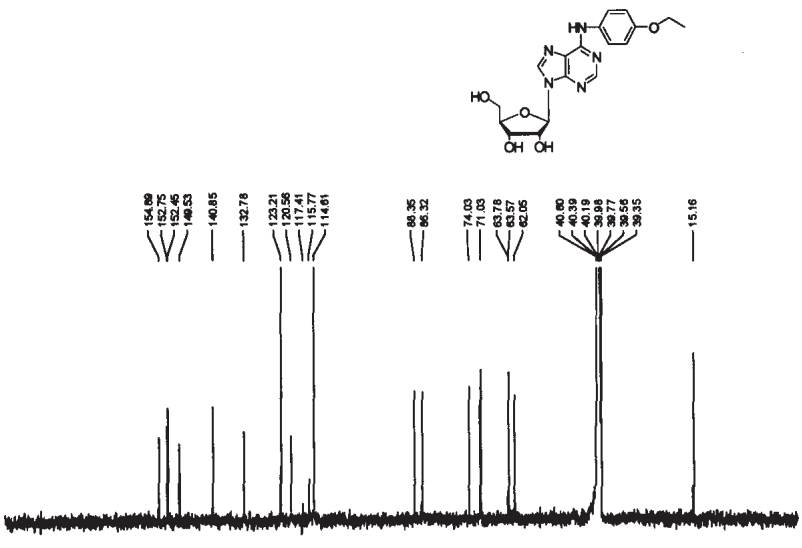

Figure S60. ${ }^{13} \mathrm{C}$ NMR of $7 \mathrm{e}$. 


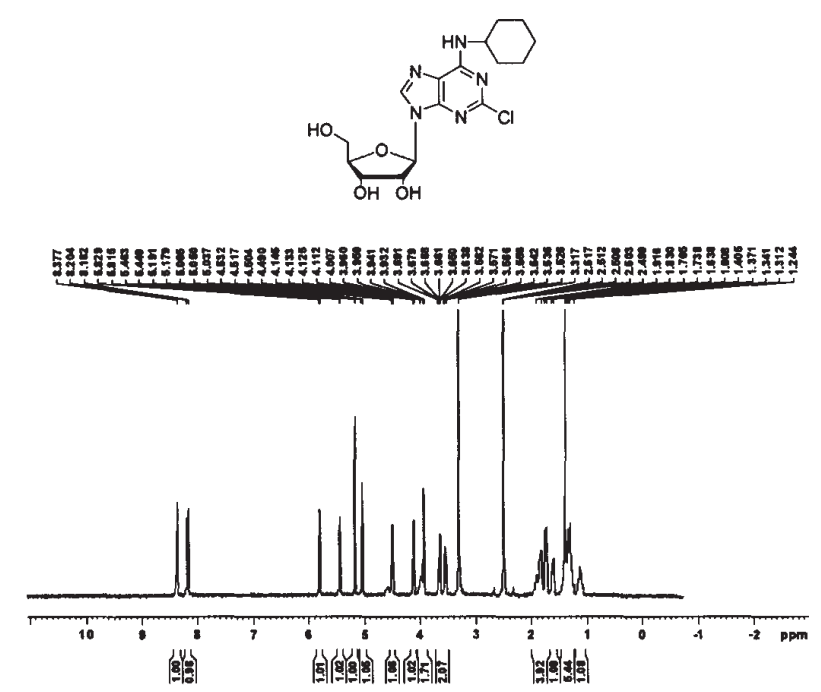

Figure S61. ${ }^{1} \mathrm{H}$ NMR of 8 .

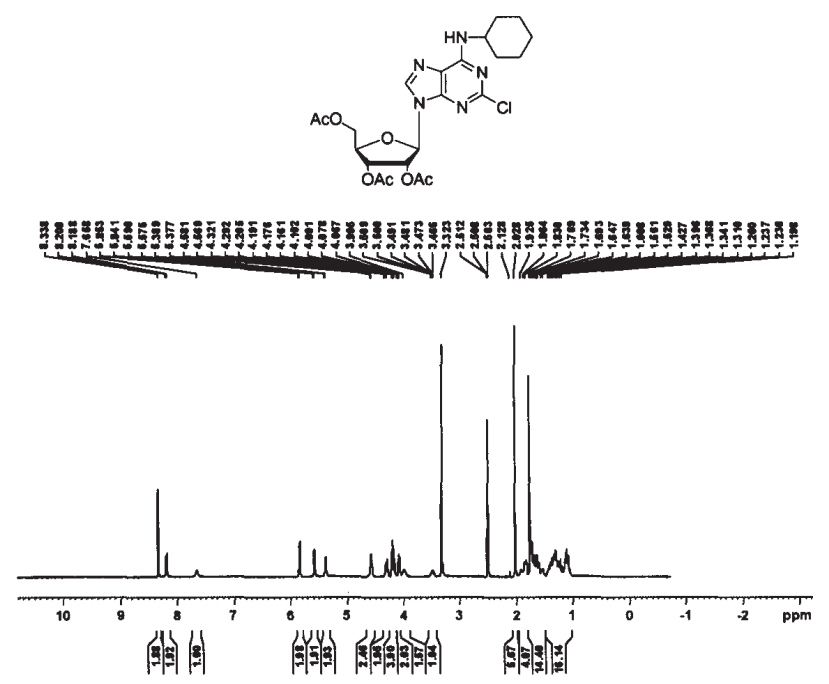

Figure S62. ${ }^{1} \mathrm{H}$ NMR of $\mathbf{8 a}^{\prime}$.

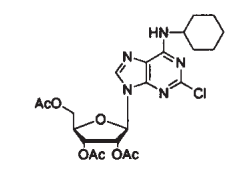

II

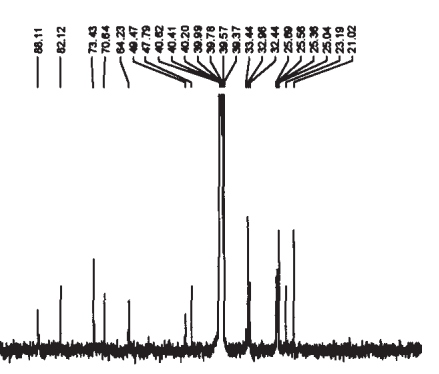

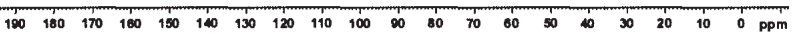

Figure $\mathbf{S 6 3} .{ }^{13} \mathrm{C}$ NMR of $8 \mathrm{a}$.

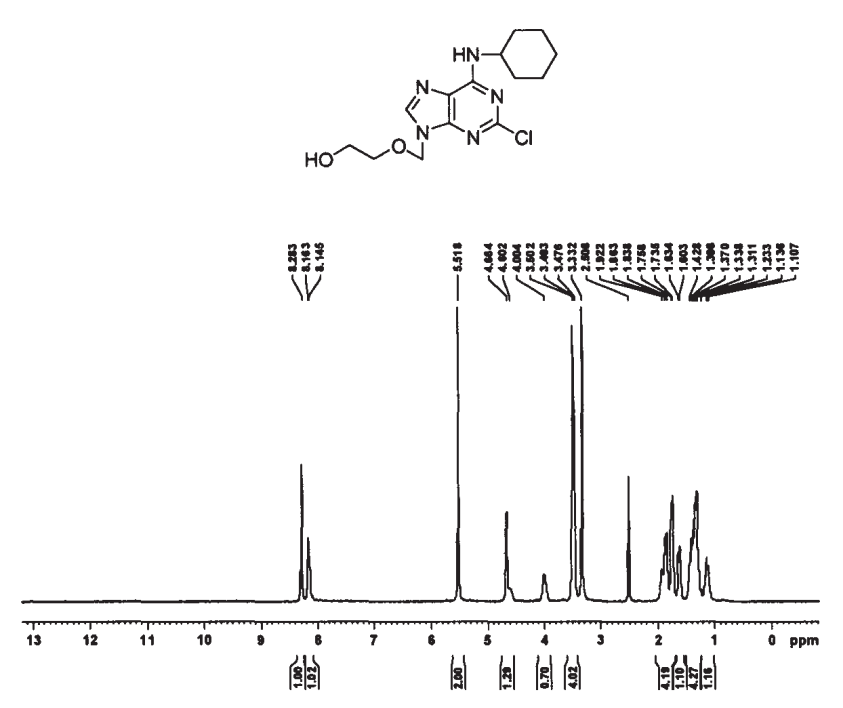

Figure S64. ${ }^{1} \mathrm{H}$ NMR of 9a.

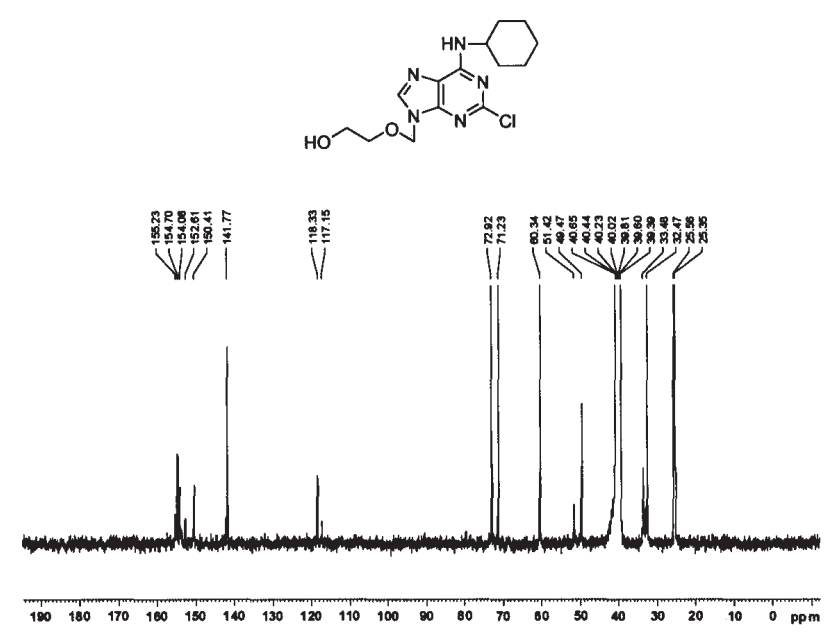

Figure S65. ${ }^{13} \mathrm{C}$ NMR of 9 a.

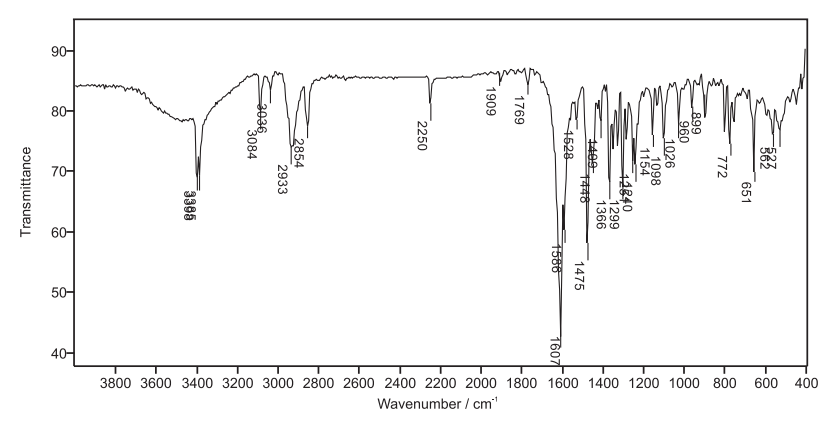

Figure S66. IR of 4a. 


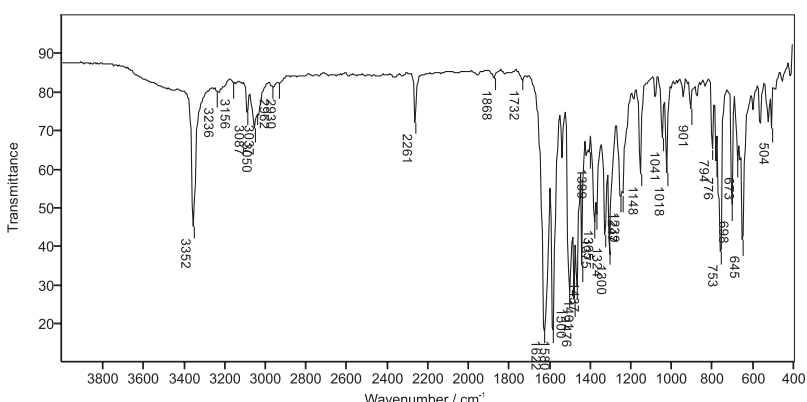

Figure S67. IR of $\mathbf{4 b}$.

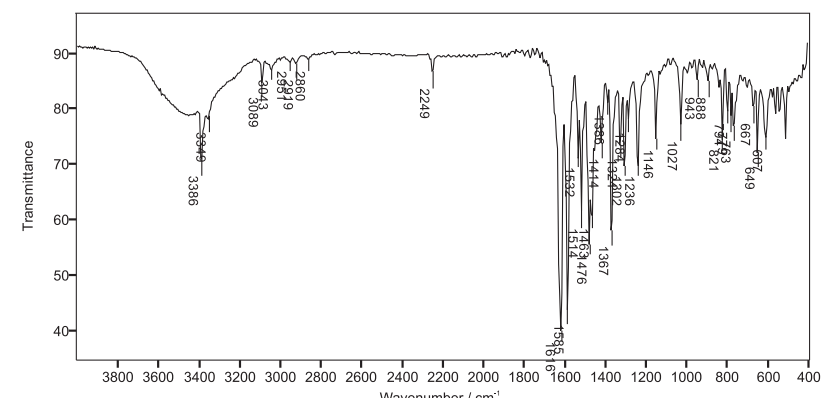

Figure S68. IR of 4c.

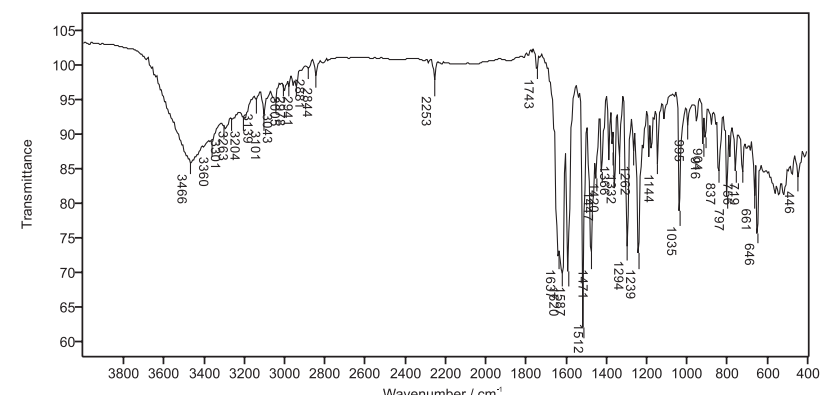

Figure S69. IR of 4d.

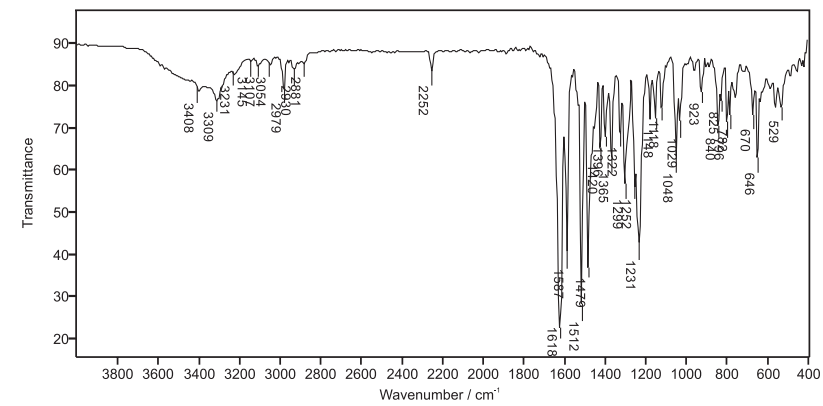

Figure S70. IR of $4 e$.

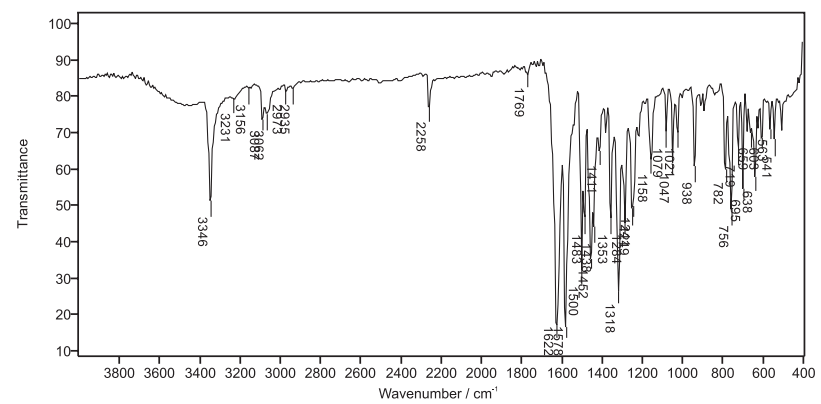

Figure S71. IR of $\mathbf{5 b}$.

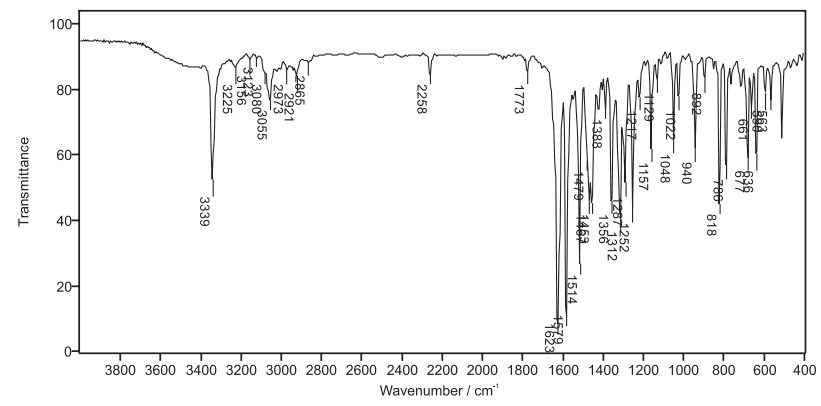

Figure S72. IR of 5c.

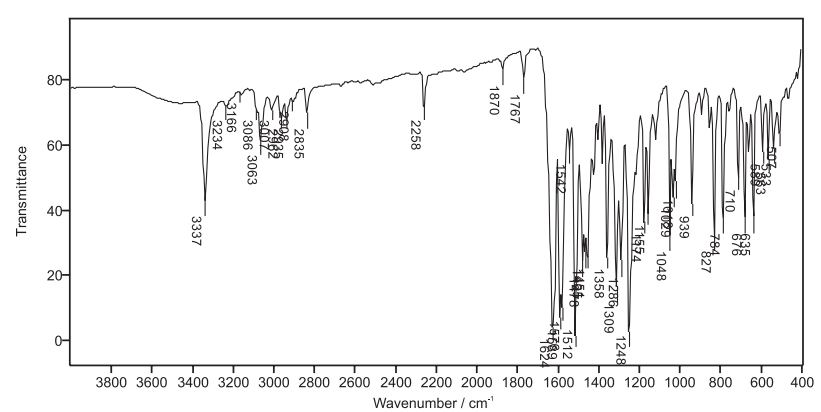

Figure S73. IR of $\mathbf{5 d}$.

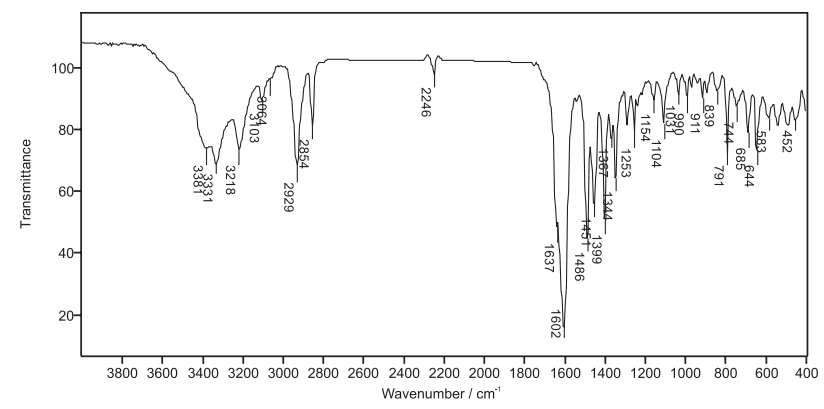

Figure S74. IR of $6 a$. 


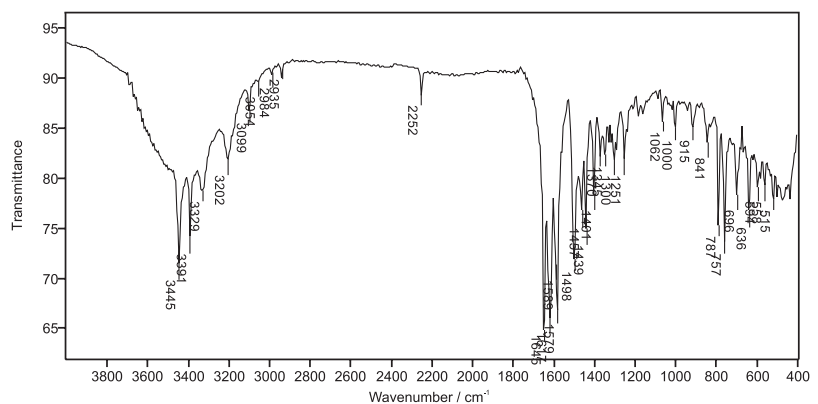

Figure S75. IR of $6 b$.

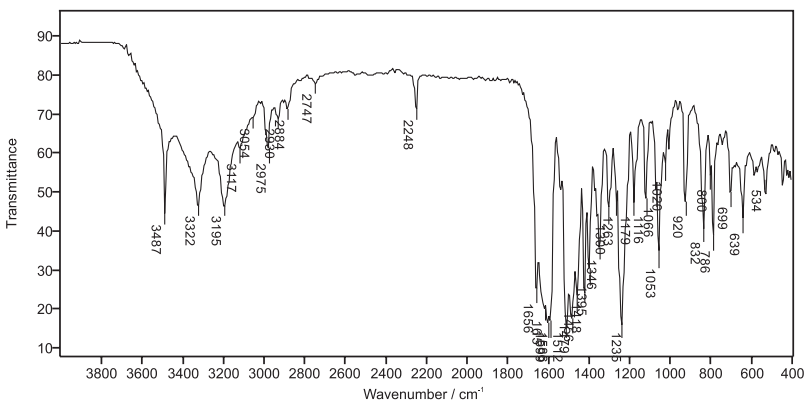

Figure S78. IR of $6 \mathrm{e}$.

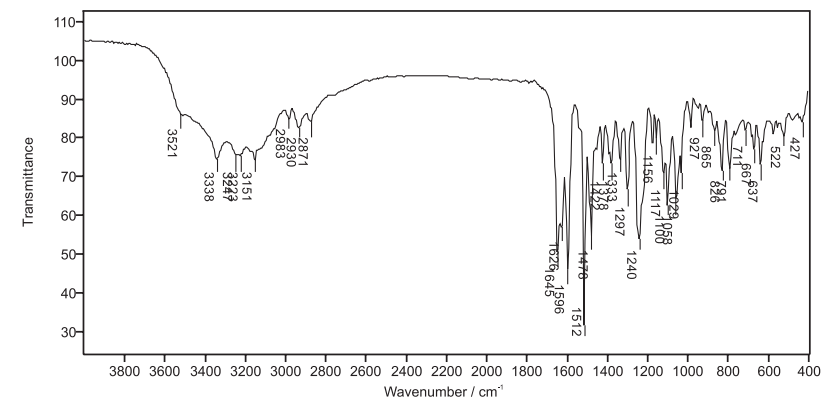

Figure S79. IR of 7e.

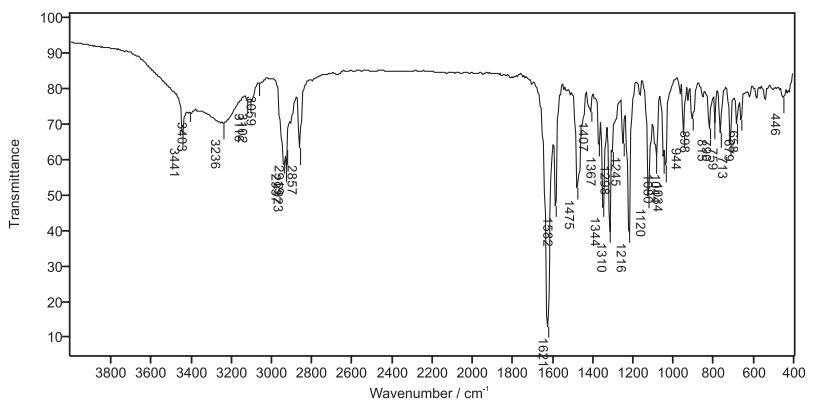

Figure S80. IR of 9a.
Figure S77. IR of 6d.

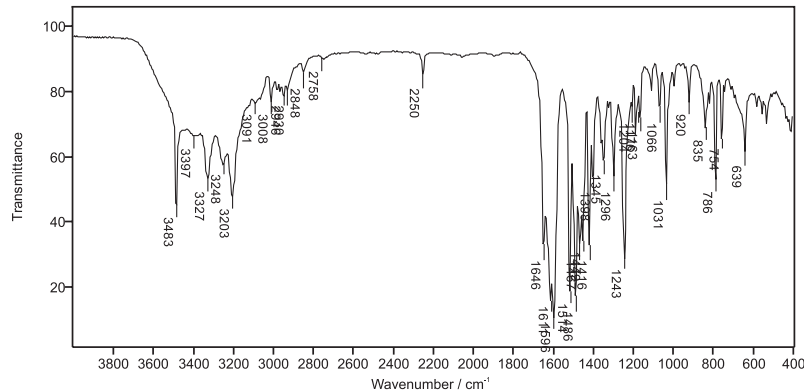

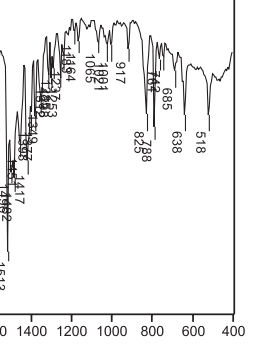

Figure S76. IR of 6c. 